\title{
Axial gravity, massless fermions and trace anomalies
}

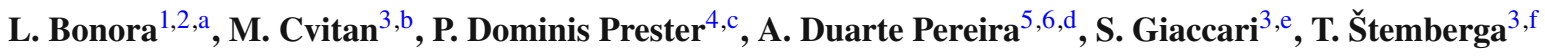 \\ ${ }^{1}$ International School for Advanced Studies (SISSA), Via Bonomea 265, 34136 Trieste, Italy \\ 2 INFN, Sezione di Trieste and KEK Theory Center, KEK, Tsukuba, Japan \\ ${ }^{3}$ Department of Physics, Faculty of Science, University of Zagreb, Bijenička cesta 32, 10000 Zagreb, Croatia \\ ${ }^{4}$ Department of Physics, University of Rijeka, Radmile Matejčić 2, 51000 Rijeka, Croatia \\ ${ }^{5}$ Departamento de Física Teórica, UERJ-Universidade Estadual do Rio de Janeiro, Rua São Francisco Xavier 524, Maracanã, Rio de Janeiro, RJ \\ 20550-013, Brazil \\ ${ }^{6}$ UFF-Universidade Federal Fluminense, Instituto de Física, Campus da Praia Vermelha, Avenida General Milton Tavares de Souza s/n, Niterói, \\ RJ 24210-346, Brazil
}

Received: 4 April 2017 / Accepted: 13 July 2017 / Published online: 1 August 2017

(C) The Author(s) 2017. This article is an open access publication

\begin{abstract}
This article deals with two main topics. One is odd parity trace anomalies in Weyl fermion theories in a $4 \mathrm{~d}$ curved background, the second is the introduction of axial gravity. The motivation for reconsidering the former is to clarify the theoretical background underlying the approach and complete the calculation of the anomaly. The reference is in particular to the difference between Weyl and massless Majorana fermions and to the possible contributions from tadpole and seagull terms in the Feynman diagram approach. A first, basic, result of this paper is that a more thorough treatment, taking account of such additional terms and using dimensional regularization, confirms the earlier result. The introduction of an axial symmetric tensor besides the usual gravitational metric is instrumental to a different derivation of the same result using Dirac fermions, which are coupled not only to the usual metric but also to the additional axial tensor. The action of Majorana and Weyl fermions can be obtained in two different limits of such a general configuration. The results obtained in this way confirm the previously obtained ones.
\end{abstract}

\section{Introduction}

This article deals with two main topics. One is odd parity trace anomalies in chiral fermion theories in a $4 d$ curved

\footnotetext{
a e-mail: bonora@sissa.it

be-mail: mcvitan@phy.hr

c e-mail: pprester@phy.uniri.hr

de-mail: duarte763@gmail.com

e e-mail: sgiaccari@phy.hr

f e-mail: tstember@phy.hr
}

background, the second is the introduction of axial gravity. The first subject has been already treated in [1,2]. The second, to our best knowledge, is new. The motivation for reconsidering the former is to clarify the theoretical background underlying the approach and complete the calculation of the anomaly, also in view of more recent results, [3]. For some aspects of the calculations in $[1,2]$ were left implicit. We refer to the possible contributions from tadpole and seagull terms in the Feynman diagram approach used there. Here we treat them explicitly. In this paper we use dimensional regularization, deferring to another paper the discussion of other regularizations. A first, basic, result of this paper is that a more thorough treatment, taking account of such additional terms, confirms the result of [1].

The second topic is motivated as follows. It is well known that in anomaly calculations the functional integral measure plays a basic role. In the case of chiral fermions the definition of such a measure is a long-standing and unsolved problem. One can bypass it by using Feynman diagram techniques, where the fermion path integral measure does not play a direct role. However, there is a way to carry out the same calculation on a theory of Dirac fermions, so that no fastidious objections can be raised about the fermion functional integral measure. Here is where the axial metric intervenes. The idea is to follow the method used in [4] for chiral gauge anomalies. It is possible to compute covariant and consistent anomalies in a unique model by coupling Dirac fermions also to an axial potential $A$, beside the familiar vector potential $V$. The anomalies one obtains in this way satisfy the Wess-Zumino consistency conditions, but depend on two potentials. The covariant anomaly for Dirac fermions coupled to $V$ alone are obtained by simply setting $A=0$. The consistent anomaly of chiral fermions coupled to $V$ are obtained by taking the limit 
$V \rightarrow V / 2, A \rightarrow V / 2$. Transposing this technique to the problem of trace anomalies for chiral fermions, requires the introduction of an axial tensor $f_{\mu \nu}$, which with some abuse of language we call metric too, besides the usual metric $g_{\mu \nu}$. This second tensor is called axial because it couples axially to Dirac fermions. The second important result of our paper is that we succeed in introducing this bimetric system, and through it we are able to derive the trace anomalies for Dirac, Majorana and Weyl fermions as particular cases of the general case. Using again dimensional regularization, we obtain in this way a confirmation of the previous, together with new, results. We suggest also an explanation for the claimed disagreement with Ref. [3].

The calculations presented here have a more general motivation, stemming from a more basic question concerning massless fermions. More precisely the question we would like to be able to answer is: is there at present a consistent field theory of massless fermions in a curved background? A massless Dirac fermion is not a good candidate in this sense, because it admits a mass term that can arise from renormalization, even if it is not initially present in the action. So the choice must be restricted to Weyl and Majorana. Also a Majorana fermion can have mass, but if its bare mass is zero, a (rigid) chiral symmetry could in principle protect this vanishing mass. However, this symmetry is anomalous on a curved background, due to the Kimura-Delbourgo-Salam anomaly, [5-7]. A Weyl fermion is certainly massless and no bare mass term exists that can threaten this property. The odd parity trace anomaly found in [1] is a new and perhaps useful aspect [8] as long as we consider the theory an effective one. However, unitarity of the theory might be imperiled in a fully quantized gravity theory interacting with chiral fermions. As pointed out in [1] this may have important implications for the existence of massless neutrinos, unless some innovative theory is introduced in order to describe truly massless fermions on a curved background.

Given the importance of this theme, we intend to return to the analysis of the odd parity trace anomaly in the presence of a gravitational background by means of other methods and other regularizations, which we believe will confirm the results obtained with the dimensional regularization.

The paper is organized as follows. In Sect. 2 we review the properties of massless Weyl and Majorana fermions in $4 d$. In Sect. 3 the anomaly derivation of [1] is reviewed and integrated with the calculation of the relevant tadpoles and seagull terms. In Sect. 4 such a revisiting is completed with the evaluation of the Ward identity for diffeomorphisms. Section 5 contains an additional discussion of the odd trace anomaly. In Sect. 6 we introduce the formalism for a MAT (metric-axial-tensor) gravity, and in Sect. 7 we couple it to Dirac fermions. Then we present a simplified derivation of the trace anomalies in such a model, and then we compute in detail the collapsing limit, which allows us to calculate the trace anomalies in an ordinary gravity background for Dirac, Weyl and Majorana fermions. Section 8 is devoted to a justification of the simplifications of the previous section. Section 9 is the conclusion. The evaluation of the triangle diagram for odd trace anomaly is shown in Appendix A. The derivation of Feynman rules in an ordinary gravity and MAT background, together with the relevant Ward identities are collected in Appendix B. The most encumbering diagram calculations can be found in Appendix C.

Notation We use a metric $g_{\mu \nu}$ with mostly - signature. The gamma matrices satisfy $\left\{\gamma^{\mu}, \gamma^{\nu}\right\}=2 g^{\mu \nu}$ and

$\gamma_{\mu}^{\dagger}=\gamma_{0} \gamma_{\mu} \gamma_{0}$

The generators of the Lorentz group are $\Sigma_{\mu \nu}=\frac{1}{4}\left[\gamma_{\mu}, \gamma_{\nu}\right]$. The charge conjugation operator $C$ is defined to satisfy

$\gamma_{\mu}^{T}=-C^{-1} \gamma_{\mu} C, \quad C C^{*}=-1, \quad C C^{\dagger}=1$

The chiral matrix $\gamma_{5}=i \gamma^{0} \gamma^{1} \gamma^{2} \gamma^{3}$ has the properties

$\gamma_{5}^{\dagger}=\gamma_{5}, \quad\left(\gamma_{5}\right)^{2}=1, \quad C^{-1} \gamma_{5} C=\gamma_{5}^{T}$

\section{Dirac, Majorana and Weyl fermions in 4d}

We would like to devote this section to a discussion of the statement that a massless Majorana fermion is the same as a Weyl fermion. The reason is that, if it is true at both classical and quantum level, there is no chance for an odd parity trace anomaly to exist and no motivation for this paper. On the other hand this statement is not undisputed. As one can easily experience, there is no well-defined or generally accepted doctrine about the properties of the quantum theories of massless Majorana and Weyl fermions. Our aim here is to examine various aspects of the problem and bring to light all the classical and quantum differences between the two types of fermions. We would like to convince the reader that there is no a priori uncontroversial evidence that the statement is true, and therefore it is prudent to leave the last word to explicit computations, such as the one for odd parity trace anomaly.

We start from a few basic facts about fermions in 4d. Let us start from a four-component Dirac fermion $\psi$. Under Lorentz it transforms as

$\psi(x) \rightarrow \psi^{\prime}\left(x^{\prime}\right)=\exp \left[-\frac{1}{2} \lambda^{\mu \nu} \Sigma_{\mu \nu}\right] \psi(x)$,

for $x^{\prime \mu}=\left(e^{\lambda}\right)^{\mu}{ }_{\nu} x^{\nu}$, where $\Sigma_{\mu \nu}=\frac{1}{4}\left[\gamma_{\mu}, \gamma_{\nu}\right]$. The Lagrangian for a free Dirac field is well known:

$i \bar{\psi} \gamma^{\mu} \partial_{\mu} \psi$.

What is often forgotten is that, like for the kinetic term of any field theory, it can be constructed because, in the spinor space, 
there exists a Lorentz invariant scalar product $\left(\Psi_{1}, \Psi_{2}\right)=$ $\left\langle\Psi_{1}^{\dagger}\left|\gamma^{0}\right| \Psi_{2}\right\rangle$. So that (3) can also be written as

$i(\psi, \gamma \cdot \partial \psi)$.

A Dirac fermion admits a Lorentz invariant mass term $m \bar{\psi} \psi=m(\psi, \psi)$.

A Dirac fermion can be seen as the sum of two Weyl fermions

$\psi_{L}=P_{L} \psi, \quad \psi_{R}=P_{R} \psi, \quad$ where $P_{L}=\frac{1+\gamma_{5}}{2}$,

$P_{R}=\frac{1-\gamma_{5}}{2}$

with opposite chiralities

$\gamma_{5} \psi_{L}=\psi_{L}, \quad \gamma_{5} \psi_{R}=-\psi_{R}$.

A left-handed Weyl fermion admits a Lagrangian kinetic term

$i\left(\psi_{L}, \gamma \cdot \partial \psi_{L}\right)=i \bar{\psi}_{L} \gamma^{\mu} \partial_{\mu} \psi_{L}$

but not a mass term, because $\left(\psi_{L}, \psi_{L}\right)=0$, since $\gamma_{5} \gamma^{0}+$ $\gamma^{0} \gamma_{5}=0$. So a Weyl fermion is massless and this property is protected by its being chiral.

In order to introduce Majorana fermions we need the notion of Lorentz covariant conjugate spinor, $\hat{\psi}$ :

$\hat{\psi}=\gamma_{0} C \psi^{*}$.

It is not hard to show that if $\psi$ transforms like (2), then

$\hat{\psi}(x) \rightarrow \hat{\psi}^{\prime}\left(x^{\prime}\right)=\exp \left[-\frac{1}{2} \lambda^{\mu \nu} \Sigma_{\mu \nu}\right] \hat{\psi}(x)$.

Therefore it makes sense to impose on $\psi$ the condition

$\psi=\hat{\psi}$

because both sides transform in the same way. A spinor satisfying (8) is, by definition, a Majorana spinor.

A Majorana spinor admits both kinetic and mass term, which can be written as $\frac{1}{2} \times$ those of a Dirac spinor.

In terms of Lorentz group representations we can summarize the situation as follows. $\gamma_{5}$ commutes with Lorentz transformations $\exp \left[-\frac{1}{2} \lambda^{\mu \nu} \Sigma_{\mu \nu}\right]$. So do $P_{L}$ and $P_{R}$. This means that the Dirac representation is reducible and multiplying the spinors by $P_{L}$ and $P_{R}$ identifies irreducible representations, the Weyl ones. To be more precise, the Weyl representations are irreducible representations of the group $S L(2, C)$, which is the covering group of the proper ortochronous Lorentz group. They are usually denoted $\left(\frac{1}{2}, 0\right)$ and $\left(0, \frac{1}{2}\right)$ in the $S U(2) \times S U(2)$ labeling of the $S L(2, C)$ irreps. As we have seen in (7), Lorentz transformations commute also with the charge conjugation operation

$\mathcal{\psi} \psi \mathcal{C}^{-1}=\eta_{C} \gamma_{0} C \psi^{*}$ where $\eta_{C}$ is a phase which, for simplicity, we set equal to 1. This also says that Dirac spinors are reducible and suggests another way to reduce them: by imposing (8) we single out another irreducible representation, the Majorana one. The Majorana representation is the minimal irreducible representation of a (one out of eight) covering of the complete Lorentz group, $[9,10]$. It is evident, and well known, that Majorana and Weyl representations are incompatible (in 4d). ${ }^{1}$

Let us recall the properties of a Weyl fermion under charge conjugation and parity. We have

$\mathcal{C} \psi_{L} \mathrm{C}^{-1}=P_{L} \mathcal{C} \psi \mathcal{C}^{-1}=P_{L} \hat{\psi}=\hat{\psi}_{L}$.

The charge conjugate of a Majorana field is itself, by definition. While the action of a Majorana field is invariant under charge conjugation, the action of a Weyl fermion is, so to say, maximally non-invariant, for

$$
\begin{aligned}
\mathcal{C}\left(\int i \overline{\psi_{L}} \gamma^{\mu} \partial_{\mu} \psi_{L}\right) \mathcal{C}^{-1} & =\int i \overline{\hat{\psi}_{L}} \gamma^{\mu \dagger} \partial_{\mu} \hat{\psi}_{L} \\
& =\int i \overline{\psi_{R}} \gamma^{\mu} \partial_{\mu} \psi_{R} .
\end{aligned}
$$

The parity operation is defined by

$\mathcal{P} \psi_{L}(t, \vec{x}) \mathcal{P}^{-1}=\eta_{P} \gamma_{0} \psi_{R}(t,-\vec{x})$

where $\eta_{P}$ is a phase. In terms of the action we have

$\mathcal{P}\left(\int \bar{\psi}_{L} \gamma^{\mu} \partial_{\mu} \psi_{L}\right) \mathcal{P}^{-1}=\int \bar{\psi}_{R} \gamma^{\mu} \partial_{\mu} \psi_{R}$,

while for a Majorana fermion the action is invariant under parity.

If we consider $\mathrm{CP}$, the action of a Majorana fermion is obviously invariant under it. For a Weyl fermion we have

$$
\begin{aligned}
\mathcal{C P} \psi_{L}(t, \vec{x})(\mathrm{eP})^{-1} & =\gamma_{0} \widehat{\psi}_{L}(t,-\vec{x})=\gamma_{0} P_{R} \hat{\psi}(t,-\vec{x}) \\
& =\gamma_{0} \hat{\psi}_{R}(t,-\vec{x})
\end{aligned}
$$

Applying CP to the Weyl action one gets

$$
\begin{aligned}
\operatorname{eP} & \left(\int i \overline{\psi_{L}} \gamma^{\mu} \partial_{\mu} \psi_{L}\right)(\mathcal{C P})^{-1} \\
& =\int i \overline{\hat{\psi}_{R}}(t,-\vec{x}) \gamma^{\mu \dagger} \partial_{\mu} \hat{\psi}_{R}(t,-\vec{x}) \\
& =\int i \overline{\hat{\psi}_{R}}(t, \vec{x}) \gamma^{\mu} \partial_{\mu} \hat{\psi}_{R}(t, \vec{x}) .
\end{aligned}
$$

But one can easily prove that

$$
\int i{\hat{\psi_{R}}}(t, \vec{x}) \gamma^{\mu} \partial_{\mu} \hat{\psi}_{R}(t, \vec{x})=\int i \overline{\psi_{L}}(x) \gamma^{\mu} \partial_{\mu} \psi_{L}(x) \text {. }
$$

\footnotetext{
${ }^{1}$ One can express the components of Majorana fermion as linear combinations of those of a Weyl fermion and vice versa; see for instance [11]. However, this does not respect the irrep decomposition.
} 
Therefore the action for a Weyl fermion is CP invariant. It is also, separately, T invariant, and, so, CPT invariant.

Now let us go to the quantum interpretation of the field $\psi_{L}$. It has the plane wave expansion

$\psi_{L}(x)=\int \mathrm{d} p\left(a(p) u_{L}(p) e^{-i p x}+b^{\dagger}(p) v_{L}(p) e^{i p x}\right)$

where $u_{L}, v_{L}$ are fixed and independent left-handed spinors (there are only two of them). The interpretation is: $b^{\dagger}(p)$ creates a left-handed particle while $a(p)$ destroys a lefthanded particle with negative helicity (because of the opposite momentum). However, Eqs. (14) and (15) force us to identify the latter with a right-handed antiparticle: $\mathrm{C}$ maps particles to antiparticles, while $\mathrm{P}$ invert helicities, so CP maps left-handed particles to right-handed antiparticles. It goes without saying that no right-handed particles or left-handed antiparticles enter the game.

Remark A mass term $\bar{\psi} \psi$ for a Dirac spinor can also be rewritten by projecting the latter into its chiral components

$\bar{\psi} \psi=\overline{\psi_{L}} \psi_{R}+\overline{\psi_{R}} \psi_{L}$.

If $\psi$ is a Majorana spinor this can be written

$\bar{\psi} \hat{\psi}=\overline{\hat{\psi}_{L}} \psi_{R}+\overline{\psi_{R}} \hat{\psi}_{L}$,

which is therefore well defined and Lorentz invariant by construction. Now, using the Lorentz covariant conjugate we can rewrite (19) as

$\left(\psi_{L}\right)^{T} C^{-1} \psi_{L}+\psi_{L}^{\dagger} C\left(\psi_{L}\right)^{*}$,

which is expressed only in terms of $\psi_{L}$. Equation (20) may create the illusion that there exists a mass term also for Weyl fermions. But this is not the case. If we add this term to the kinetic term (5) we obtain an action whose equations of motion have $m C{\overline{\psi_{L}}}^{T}$ as the mass term. These involve both chiralities as a consequence of the self-adjointness of (20). This implies that there does not exist such a thing as a "massive Weyl propagator", i.e. a massive propagator involving only one chirality, which, in particular, renders the use of the Pauli-Villars regularization problematic. ${ }^{2}$ Another possibility could be to write down a massive Dirac equation of motion for a Weyl fermion,

$i \gamma^{\mu} \partial_{\mu} \psi_{L}-m \psi_{L}=0$

but this equation breaks Lorentz covariance because the first piece transforms according to a right-handed representation while the second according to a left-handed one, and is not

\footnotetext{
2 Sometimes a Dirac or Majorana propagator is used in its place. A minimal precaution, in such a case, would be to check the results obtained with this regularization by comparing them with those obtained with others.
}

Lagrangian. The reason is, of course, that (20) is not expressible in the same canonical form as (5). This structure is clearly visible in the four component formalism used so far, much less recognizable in the two-component formalism.

The fact that a massive Majorana fermion and a Weyl fermion are different objects is, in our opinion, uncontroversial. The question whether a massless Majorana fermion is or is not the same as a Weyl fermion at both classical and quantum level, as we pointed out above, is not so clearly established. Let us consider the case in which there is no quantum number appended to the fermions. The reason why they are sometimes considered as a unique object is due, we think, to the fact that we can establish a one-to-one correspondence between the components of a Weyl spinor and those of a Majorana spinor in such a way that the Lagrangian (particularly in two-component notation) looks the same. In fact this is not decisive, as we will see in a moment. But let us mention first the evident differences between the two. The first, and most obvious, is the one we have already mentioned: they belong to two different representations of the Lorentz group, irreducible to each other (it should be standard lore that in $4 \mathrm{~d}$ there cannot exist a spinor that is simultaneously Majorana and Weyl). Another macroscopic difference is that the helicity of a Weyl fermion is well defined and corresponds to its chirality, while the chirality of a Majorana fermion is undefined, so that the relation with its helicity is also undefined. Next, a parity operation maps the Majorana action into itself, while it maps the Weyl action (5) into the same action for the opposite chirality. Same for the charge conjugation operator. Finally, going to the quantum theories, the fermion path integral measures in the two cases are different. This is the crucial point as far as the matter discussed in this paper is concerned, i.e. anomalies. Let us expand on it. The path integral of a free Dirac fermion (3) is interpreted as the determinant of the massless Dirac operator $\not D=i \not \partial+V$ (where $V$ denotes any potential), i.e. the (suitably regularized) product of its eigenvalues. A similar interpretation holds for a massless Majorana fermion, while for a Weyl fermion it is not so straightforward. Since the Dirac operator anticommutes with $\gamma_{5}$, it maps a left-handed spinor to a right-handed one. Therefore the eigenvalue problem is not even defined for $\not D_{L}=\not D P_{L}$. We may replace the looked for det $\not D_{L}$ with $\left(\operatorname{det}\left(\not D_{L}^{\dagger} \not D_{L}\right)\right)^{\frac{1}{2}}$. But in this case we have to face the problem of an undetermined overall phase factor. This impasse has been known for a long time. ${ }^{3}$ A few

\footnotetext{
${ }^{3}$ It is well known that in particular this prevents using the Fujikawa method for chiral theories, because the latter, at least in its original form, holds when in the theory both chiralities are present. This problem has been discussed in detail in [12], with explicit examples: it is shown there that the original Fujikawa method cannot reproduce the non-Abelian consistent chiral anomalies, but only the covariant ones
} 
ways have been devised to overcome it. One is to use a perturbative approach, via the Feynman diagram technique, in a chiral fermion theory, wherein Lorentz covariance is taken into account via the chiral vertices. This is the method used in $[1,2]$. We will revisit it below. Later on (in Part II) we will consider another approach, based on Dirac fermions, $[4,12,13]$, (i.e. with the ordinary Dirac path integral measure), whereby the chiral fermion theory is recovered as a limiting case. Finally, although we do not use it here, we should mention the method recently devised in [14], where a fifth dimension is introduced as a regulator.

We think the above arguments are more than enough to conclude that massless Majorana and Weyl fermions, notwithstanding some similarities, may really be different objects. However, to conclude, it is worth trying to counter a common misconception that comes from what we said above: we can establish a one-to-one correspondence between the components of a Weyl spinor and those of a Majorana spinor in such a way that the Lagrangians in two-component notation look the same. If, for instance, in the chiral representation we represent $\psi_{L}$ as $\left(\begin{array}{c}\omega \\ 0\end{array}\right)$, where $\omega$ is a two component spinor, then (5) above becomes

$i \omega^{\dagger} \bar{\sigma}^{\mu} \partial_{\mu} \omega$

which has the same form as a massless Majorana action. Now, if the action is the same for both Weyl and Majorana, how can there be differences? This (problematic) syllogism may cause gross misunderstandings. Well, first, in general, the action of a physical system does not contain all the information concerning the system, there being specifications that have to be added separately. Second, even though numerically the actions coincide, the way the actions respond to a variation of the Weyl and Majorana field is different. One leads to the Weyl equation of motion, the other to the Majorana one. The delicate point is precisely this: when we take the variation of an action with respect to a field in order to extract the equations of motion, we have to make sure that

Footnote 3 continued

in chirally symmetric theories. It follows that one cannot expect to be able to reproduce the odd parity trace anomaly in a left-handed theory, because the latter belongs to the same class as the non-Abelian consistent chiral anomalies, that is, the class of anomalies having opposite sign for opposite chirality. This remark applies to [3], which, following the method of Fujikawa and using Pauli-Villars regularization, obtains a vanishing odd trace anomaly and seems to contradict our result below. Although we intend to return more punctually on this issue, let us point out for the time being that using a Dirac fermion path integration measure amounts to introducing in the game both chiralities, even though formally the action is declared to be the Weyl one. We have seen that the classical action can take various forms, but for this anomaly what matters is that only one chirality is involved through all the steps, including the path integration measure. Therefore, we believe, the result of [3] applies to Dirac and Majorana fermions and is in fact consistent with ours. the variations do not break the symmetries or the properties we wish to be present in the equations of motion. In general, we do this automatically, without thinking of it. ${ }^{4}$ But in this case more than the normal care has to be used. If we wish the EoM to preserve chirality we must use variations that preserve chirality, i.e. must be eigenfunctions of $\gamma_{5}$. If instead we wish the EoM to transform in the Majorana representation we have to use variations that transform suitably, i.e. must be eigenfunctions of the charge conjugation operation. If we do so we o btain two different results, which are irreducible to each other, no matter what action we use. ${ }^{5}$ Third, and most important, as already pointed out, in the quantum theory a crucial role is played by the functional measure, which is very likely to be different for Weyl and Majorana fermions.

Concluding this introductory discussion, we think the identification of a Weyl fermion with a massless Majorana one should not be taken as granted as sometimes stated in the literature. It is prudent to avoid a priori conclusions, but rather develop both hypotheses (not only one) and compare the end results. This said, it is important to find properties that differentiate Weyl and massless Majorana fermions. In this paper, following $[1,2]$, we show that one such property is the parity odd Weyl anomaly. The latter is 0 for a massless Majorana fermion, while it equals the Pontryagin density for a Weyl fermion (the even parity trace anomaly is the same for both).

\section{Part I}

\section{Odd parity trace anomaly in chiral theories}

In this section we reconsider the calculation of the odd trace anomaly in [1] (for an introduction to anomalies see [1517]). The motivation for this is that in [1], as well as in [2], tadpoles and seagull diagrams were disregarded. In ordinary (non-chiral) theories coupled to gravity such diagrams contribute local terms to the effective action, and help restoring conservation, which otherwise would be violated by local terms, [18]. As we shall see below, these diagrams are instead ineffective for the parity odd diagrams in a chiral theory, and do not change the final result. However, a complete treatment demands that they should be taken into account and evaluated.

\footnotetext{
${ }^{4}$ For instance, in gravity theories, the metric variation $\delta g_{\mu \nu}$ is generic while not ceasing to be a symmetric tensor.

${ }^{5}$ It is clear that, eventually, all the components, both of a Weyl and a massless Majorana fermion, satisfy the massless Klein-Gordon equation. But this is not a qualifying property in this context, otherwise, for instance, any two (anticommuting) complex massless scalar fields would be the same as a Weyl fermion, and any four real massless scalar fields would be the same as a massless Majorana fermion.
} 
The model we considered in [1] was a left-handed Weyl spinor coupled to external gravity in $4 \mathrm{~d}$. The action is

$S=\int \mathrm{d}^{4} x \sqrt{|g|} i \overline{\psi_{L}} \gamma^{m}\left(\nabla_{m}+\frac{1}{2} \omega_{m}\right) \psi_{L}$

where $\gamma^{m}=e_{a}^{m} \gamma^{a}, \nabla(m, n, \ldots$ are world indices, $a, b, \ldots$ are flat indices) is the covariant derivative with respect to the world indices and $\omega_{m}$ is the spin connection:

$\omega_{m}=\omega_{m}^{a b} \Sigma_{a b}$

where $\Sigma_{a b}=\frac{1}{4}\left[\gamma_{a}, \gamma_{b}\right]$ are the Lorentz generators. Finally $\psi_{L}=\frac{1+\gamma_{5}}{2} \psi$. Classically the energy-momentum tensor

$T^{\mu \nu}=-\frac{i}{4} \overline{\psi_{L}} \gamma^{\mu} \stackrel{\leftrightarrow}{\nabla^{v}} \psi_{L}+(\mu \leftrightarrow \nu)$

is both conserved on shell and traceless.

From (23) we extracted the (simplified) Feynman rules as follows. The action (23) can be written as

$S=\int \mathrm{d}^{4} x \sqrt{|g|}\left[\frac{i}{2} \overline{\psi_{L}} \gamma^{\mu} \stackrel{\leftrightarrow}{\partial}{ }_{\mu} \psi_{L}-\frac{1}{4} \epsilon^{\mu a b c} \omega_{\mu a b} \overline{\psi_{L}} \gamma_{c} \gamma_{5} \psi_{L}\right]$

where it is understood that the derivative applies to $\psi_{L}$ and $\overline{\psi_{L}}$ only. We have used the relation $\left\{\gamma^{a}, \Sigma^{b c}\right\}=i \epsilon^{a b c d} \gamma_{d} \gamma_{5}$.

Expanding

$e_{\mu}^{a}=\delta_{\mu}^{a}+\chi_{\mu}^{a}+\cdots, \quad e_{a}^{\mu}=\delta_{a}^{\mu}+\hat{\chi}_{a}^{\mu}+\cdots$,

and $g_{\mu \nu}=\eta_{\mu \nu}+h_{\mu \nu}$

and inserting these expansions in the defining relations $e_{\mu}^{a} e_{b}^{\mu}=\delta_{b}^{a}, \quad g_{\mu \nu}=e_{\mu}^{a} e_{\nu}^{b} \eta_{a b}$, one finds

$\hat{\chi}_{v}^{\mu}=-\chi_{v}^{\mu}$ and $h_{\mu \nu}=2 \chi_{\mu \nu}$.

Expanding accordingly the spin connection

$\omega_{\mu a b}=e_{\nu a}\left(\partial_{\mu} e_{b}^{v}+e^{\sigma}{ }_{b} \Gamma_{\sigma}{ }^{\nu}{ }_{\mu}\right)$,

$\Gamma_{\sigma}^{\nu}{ }_{\mu}=\frac{1}{2} \eta^{\nu \lambda}\left(\partial_{\sigma} h_{\lambda \mu}+\partial_{\mu} h_{\lambda \sigma}-\partial_{\lambda} h_{\sigma \mu}\right)+\cdots$

after some algebra one gets

$\omega_{\mu a b} \epsilon^{\mu a b c}=-\frac{1}{4} \epsilon^{\mu a b c} \partial_{\mu} h_{a \lambda} h_{b}^{\lambda}+\cdots$.

Therefore, up to second order the action can be written (by incorporating $\sqrt{|g|}$ in a redefinition of the $\psi$ field)

$$
\begin{aligned}
S \approx & \int \mathrm{d}^{4} x\left[\frac{i}{2}\left(\delta_{a}^{\mu}-\frac{1}{2} h_{a}^{\mu}\right) \overline{\psi_{L}} \gamma^{a} \stackrel{\leftrightarrow}{\partial}{ }_{\mu} \psi_{L}\right. \\
& \left.+\frac{1}{16} \epsilon^{\mu a b c} \partial_{\mu} h_{a \lambda} h_{b}^{\lambda} \bar{\psi}_{L} \gamma_{c} \gamma_{5} \psi_{L}\right] .
\end{aligned}
$$

The free action is

$S_{\text {free }}=\int \mathrm{d}^{4} x \frac{i}{2} \overline{\psi_{L}} \gamma^{a} \stackrel{\leftrightarrow}{\partial}_{a} \psi_{L}$ and the lowest interaction terms are

$$
\begin{aligned}
S_{\mathrm{int}}= & \int \mathrm{d}^{4} x\left[-\frac{i}{4} h_{a}^{\mu} \overline{\psi_{L}} \gamma^{a} \stackrel{\leftrightarrow}{\partial}_{\mu} \psi_{L}\right. \\
& \left.+\frac{1}{16} \epsilon^{\mu a b c} \partial_{\mu} h_{a \lambda} h_{b}^{\lambda} \bar{\psi}_{L} \gamma_{c} \gamma_{5} \psi_{L}\right] .
\end{aligned}
$$

Retaining only the above terms of the action of (30), the Feynman rules are as follows (momenta are ingoing and the external gravitational field is assumed to be $h_{\mu \nu}$ ). The fermion propagator is

$P: \frac{i}{p+i \epsilon}$.

The two-fermion-one-graviton vertex is

$V_{f f h}:-\frac{i}{8}\left[\left(p+p^{\prime}\right)_{\mu} \gamma_{\nu}+\left(p+p^{\prime}\right)_{\nu} \gamma_{\mu}\right] \frac{1+\gamma_{5}}{2}$.

The two-fermion-two-graviton vertex $\left(V_{f f h h}^{\epsilon}\right)$ is

$V_{f f h h}^{\epsilon}: \frac{1}{64} t_{\mu \nu \mu^{\prime} \nu^{\prime} \kappa \lambda}\left(k-k^{\prime}\right)^{\lambda} \gamma^{\kappa} \frac{1+\gamma_{5}}{2}$

where

$t_{\mu \nu \mu^{\prime} \nu^{\prime} \kappa \lambda}=\eta_{\mu \mu^{\prime}} \epsilon_{\nu v^{\prime} \kappa \lambda}+\eta_{\nu \nu^{\prime}} \epsilon_{\mu \mu^{\prime} \kappa \lambda}+\eta_{\mu \nu^{\prime}} \epsilon_{\nu \mu^{\prime} \kappa \lambda}$

$$
+\eta_{\nu \mu^{\prime}} \epsilon_{\mu \nu^{\prime} \kappa \lambda} \text {. }
$$

\subsection{Complete expansion}

The previous action (23) is a simplified one. It disregards the measure $\sqrt{|g|}$, which is incorporated in the fermion field $\psi$. In a more complete approach one should take into account tadpole and seagull terms and reinsert $\sqrt{|g|}$ in the action. Some of these, in principle, might be relevant for the trace anomaly. To this end we need the complete expansion in $h_{\mu \nu}$ up to order three of the action, more precisely,

$$
\begin{aligned}
g_{\mu \nu}= & \eta_{\mu \nu}+h_{\mu \nu}, \\
g^{\mu \nu}= & \eta^{\mu \nu}-h^{\mu \nu}+\left(h^{2}\right)^{\mu \nu}+\cdots, \\
e_{a}^{\mu}= & \delta_{a}^{\mu}-\frac{1}{2} h_{a}^{\mu}+\frac{3}{8}\left(h^{2}\right)_{a}^{\mu}-\frac{5}{16}\left(h^{3}\right)_{a}^{\mu}+\cdots, \\
e_{\mu}^{a}= & \delta_{\mu}^{a}+\frac{1}{2} h_{\mu}^{a}-\frac{1}{8}\left(h^{2}\right)_{\mu}^{a}+\frac{1}{16}\left(h^{3}\right)_{\mu}^{a}+\cdots, \\
\sqrt{|g|}= & 1+\frac{1}{2}(\operatorname{tr} h)+\frac{1}{8}(\operatorname{tr} h)^{2}-\frac{1}{4}\left(\operatorname{tr} h^{2}\right)-\frac{1}{8}(\operatorname{tr} h)\left(\operatorname{tr} h^{2}\right) \\
& +\frac{1}{48}(\operatorname{tr} h)^{3}+\frac{1}{6}\left(\operatorname{tr} h^{3}\right)+\cdots,
\end{aligned}
$$

and

$$
\begin{aligned}
\Gamma_{\mu \nu}^{\lambda}= & \frac{1}{2}\left(\partial_{\mu} h_{\nu}^{\lambda}+\partial_{\nu} h_{\mu}^{\lambda}-\partial^{\lambda} h_{\mu \nu}\right) \\
& -\frac{1}{2}\left(h-h^{2}\right)^{\lambda \rho}\left(\partial_{\mu} h_{\rho \nu}+\partial_{\nu} h_{\rho \mu}-\partial_{\rho} h_{\mu \nu}\right) .
\end{aligned}
$$


In this approximation the spin connection is

$$
\begin{aligned}
\omega_{\mu}^{a b}= & \frac{1}{2}\left(\partial^{b} h_{\mu}^{a}-\partial^{a} h_{\mu}^{b}\right)+\frac{1}{4}\left(h^{\sigma a} \partial_{\sigma} h_{\mu}^{b}-h^{\sigma b} \partial_{\sigma} h_{\mu}^{a}\right. \\
& \left.+h^{b \sigma} \partial^{a} h_{\sigma \mu}-h^{a \sigma} \partial^{b} h_{\sigma \mu}\right) \\
& -\frac{1}{8}\left(h^{a \sigma} \partial_{\mu} h_{\sigma}^{b}-h^{b \sigma} \partial_{\mu} h_{\sigma}^{a}\right) \\
& +\frac{1}{8}\left(\left(h^{2}\right)^{a \lambda} \partial_{\mu} h_{\lambda}^{b}-\left(h^{2}\right)^{b \lambda} \partial_{\mu} h_{\lambda}^{a}\right) \\
& +\frac{3}{16}\left(\left(h^{2}\right)^{a \lambda} \partial^{b} h_{\mu \lambda}-\left(h^{2}\right)^{b \lambda} \partial^{a} h_{\mu \lambda}\right) \\
& -\frac{3}{16}\left(\left(h^{2}\right)^{a \lambda} \partial_{\lambda} h_{\mu}^{b}-\left(h^{2}\right)^{b \lambda} \partial_{\lambda} h_{\mu}^{a}\right) \\
& +\frac{1}{8}\left(h^{a \rho} h^{b \lambda}-h^{b \rho} h^{a \lambda}\right) \partial_{\lambda} h_{\mu \rho}+\cdots .
\end{aligned}
$$

Up to third order in $h$ the action is

$$
\begin{aligned}
& S=\int \mathrm{d}^{4} x\left[\frac{i}{2} \overline{\psi_{L}} \gamma^{m} \stackrel{\leftrightarrow}{\partial}_{m} \psi_{L}-\frac{i}{4} \bar{\psi}_{L} h_{a}^{m} \gamma^{a} \stackrel{\leftrightarrow}{\partial}_{m} \psi_{L}\right. \\
& +\frac{3 i}{16} \overline{\psi_{L}}\left(h^{2}\right)_{a}^{m} \gamma^{a} \stackrel{\leftrightarrow}{\partial}_{m} \psi_{L}-\frac{5 i}{32} \bar{\psi}_{L}\left(h^{3}\right)_{a}^{m} \gamma^{a} \stackrel{\leftrightarrow}{\partial}_{m} \psi_{L} \\
& -\frac{1}{16} \epsilon^{m a b c} \overline{\psi_{L}} \gamma_{c} \gamma_{5} \psi_{L}\left(h_{m}^{\sigma} \partial_{a} h_{b \sigma}+\left(h^{2}\right)_{m}^{\sigma} \partial_{b} h_{a \sigma}\right. \\
& \left.-h_{m}^{\rho} h_{a}^{\sigma} \partial_{\sigma} h_{\rho b}-\frac{1}{2} h_{m}^{\rho} \partial_{a} h_{\rho \sigma} h_{c}^{\sigma}\right) \\
& +\frac{1}{2}(\operatorname{tr} h)\left(\frac{i}{2} \overline{\psi_{L}} \gamma^{m} \stackrel{\leftrightarrow}{\partial}_{m} \psi_{L}-\frac{i}{4} \overline{\psi_{L}} h_{a}^{m} \gamma^{a} \stackrel{\leftrightarrow}{\partial}_{m} \psi_{L}\right. \\
& +\frac{3 i}{16} \overline{\psi_{L}}\left(h^{2}\right)_{a}^{m} \gamma^{a} \stackrel{\leftrightarrow}{\partial} m \psi_{L} \\
& \left.-\frac{1}{16} \epsilon^{m a b c} \overline{\psi_{L}} \gamma_{c} \gamma_{5} \psi_{L} h_{m}^{\sigma} \partial_{a} h_{b \sigma}\right) \\
& +\left(\frac{1}{8}(\operatorname{tr} h)^{2}-\frac{1}{4}\left(\operatorname{tr} h^{2}\right)\right)\left(\frac{i}{2} \overline{\psi_{L}} \gamma^{m} \stackrel{\leftrightarrow}{\partial}_{m} \psi_{L}\right. \\
& \left.-\frac{i}{4} \overline{\psi_{L}} h_{a}^{m} \gamma^{a} \stackrel{\leftrightarrow}{\partial} m \psi_{L}\right) \\
& +\left(-\frac{1}{8}(\operatorname{tr} h)\left(\operatorname{tr} h^{2}\right)+\frac{1}{48}(\operatorname{tr} h)^{3}+\frac{1}{6}\left(\operatorname{tr} h^{3}\right)\right) \\
& \left.\frac{i}{2} \overline{\psi_{L}} \gamma^{m} \stackrel{\leftrightarrow}{\partial}_{m} \psi_{L}+\cdots\right] .
\end{aligned}
$$

The propagator (31) comes from the first term of the first line in the RHS of (38). The vertex $V_{f f h}$ comes from the second term, while $V_{f f h h}^{\epsilon}$ originates from the first term in the second line of (38). There are many other vertices of the type $V_{f f h}, V_{f f h h}, V_{f f h h h}$. It is important to single out which may be relevant to trace anomalies.

The Ward identity for Weyl invariance, in the absence of anomalies, is

$$
\begin{aligned}
\mathcal{T}(x) \equiv & g_{\mu \nu}(x)\left\langle\left\langle T^{\mu \nu}(x)\right\rangle\right\rangle=\left\langle\left\langle T_{\mu}^{\mu}(x)\right\rangle\right\rangle \\
& +h_{\mu \nu}(x)\left\langle\left\langle T^{\mu \nu}(x)\right\rangle\right\rangle=0 .
\end{aligned}
$$

Writing

$$
\begin{aligned}
\left\langle\left\langle T^{\mu \nu}(x)\right\rangle\right\rangle= & \left\langle 0\left|T_{(0)}^{\mu \nu}(x)\right| 0\right\rangle \\
& +\sum_{n=1}^{\infty} \frac{1}{2^{n} n !} \int \prod_{i=0}^{n} \mathrm{~d} x_{i} h_{\mu_{1} v_{1}}\left(x_{1}\right) \ldots h_{\mu_{n} v_{n}}\left(x_{n}\right) \\
& \times \mathcal{T}^{\mu \nu \mu_{1} v_{1} \cdots \mu_{n} v_{n}}\left(x, x_{1}, \ldots, x_{n}\right),
\end{aligned}
$$

order by order in $h$, Eq. (39) breaks down to

$$
\begin{aligned}
\mathcal{T}^{(0)}(x) \equiv & \left\langle 0\left|T_{(0) \mu}{ }^{\mu}(x)\right| 0\right\rangle=0, \\
\mathcal{T}^{(1)}(x) \equiv & \mathcal{T}_{\mu}^{\mu \mu_{1} v_{1}}\left(x, x_{1}\right) \\
& +2 \delta\left(x-x_{1}\right)\left\langle 0\left|T_{(0)}^{\mu_{1} v_{1}}(x)\right| 0\right\rangle=0, \\
\mathcal{T}^{(2)}(x) \equiv & \mathcal{T}_{\mu}^{\mu \mu_{1} v_{1} \mu_{2} v_{2}}\left(x, x_{1}, x_{2}\right) \\
& +2 \delta\left(x-x_{1}\right) \mathcal{T}^{\mu_{1} v_{1} \mu_{2} v_{2}}\left(x, x_{2}\right) \\
& +2 \delta\left(x-x_{2}\right) \mathcal{T}^{\mu_{2} v_{2} \mu_{1} v_{1}}\left(x, x_{1}\right)=0,
\end{aligned}
$$

where

$$
\begin{aligned}
& T_{(0)}^{\mu \nu}=\left.2 \frac{\delta S}{\delta h_{\mu \nu}(x)}\right|_{h=0}=-\frac{i}{4}\left(\overline{\psi_{L}} \gamma^{\mu} \overleftrightarrow{\partial}^{v} \psi_{L}+\mu \leftrightarrow v\right) \\
&+\frac{i}{2} \eta_{\mu \nu} \overline{\psi_{L}} \gamma^{m} \stackrel{\leftrightarrow}{\partial}_{m} \psi_{L}, \\
& \mathcal{T}^{\mu \nu \mu_{1} v_{1}}\left(x, x_{1}\right)= i\left\langle 0\left|\mathcal{T} T_{(0)}^{\mu \nu}(x) T_{(0)}^{\mu_{1} \nu_{1}}\left(x_{1}\right)\right| 0\right\rangle \\
&-\eta^{\mu_{1} \nu_{1}} \delta\left(x-x_{1}\right)\left\langle 0\left|T_{(0)}^{\mu \nu}(x)\right| 0\right\rangle \\
&+4\left\langle 0\left|\frac{\delta^{2} S}{\delta h_{\mu \nu}(x) \delta h_{\mu_{1} v_{1}}\left(x_{1}\right)}\right| 0\right\rangle,
\end{aligned}
$$

and

$$
\begin{aligned}
& \mathcal{T}^{\mu \nu \mu_{1} \nu_{1} \mu_{2} \nu_{2}}\left(x, x_{1}, x_{2}\right) \\
& =-\left\langle 0\left|\mathcal{T} T_{(0)}^{\mu \nu}(x) T_{(0)}^{\mu_{1} v_{1}}\left(x_{1}\right) T_{(0)}^{\mu_{2} \nu_{2}}\left(x_{2}\right)\right| 0\right\rangle \\
& +4 i\left\langle 0\left|\mathcal{T} T_{(0)}^{\mu \nu}(x) \frac{\delta^{2} S}{\delta h_{\mu_{1} \nu_{1}}\left(x_{1}\right) \delta h_{\mu_{2} \nu_{2}}\left(x_{2}\right)}\right| 0\right\rangle \\
& -i \eta^{\mu_{1} v_{1}} \delta\left(x-x_{1}\right)\left\langle 0\left|\mathcal{T} T_{(0)}^{\mu v}(x) T_{(0)}^{\mu_{2} v_{2}}\left(x_{2}\right)\right| 0\right\rangle \\
& -i \eta^{\mu_{2} \nu_{2}} \delta\left(x-x_{2}\right)\left\langle 0\left|\mathcal{T} T_{(0)}^{\mu \nu}(x) T_{(0)}^{\mu_{1} v_{1}}\left(x_{1}\right)\right| 0\right\rangle \\
& +4 i\left\langle 0\left|\mathcal{T} T_{(0)}^{\mu_{1} \nu_{1}}\left(x_{1}\right) \frac{\delta^{2} S}{\delta h_{\mu \nu}(x) \delta h_{\mu_{2} \nu_{2}}\left(x_{2}\right)}\right| 0\right\rangle \\
& +4 i\left\langle 0\left|\mathcal{T} T_{(0)}^{\mu_{2} \nu_{2}}\left(x_{2}\right) \frac{\delta^{2} S}{\delta h_{\mu_{1} \nu_{1}}\left(x_{1}\right) \delta h_{\mu \nu}(x)}\right| 0\right\rangle \\
& +\left(\eta^{\mu_{1} \nu_{1}} \eta^{\mu_{2} \nu_{2}}+\eta^{\mu_{1} \nu_{2}} \eta^{\mu_{2} \nu_{1}}+\eta^{\mu_{1} \mu_{2}} \eta^{\nu_{1} \nu_{2}}\right) \\
& \times \delta\left(x-x_{1}\right) \delta\left(x-x_{2}\right)\left\langle 0\left|T_{(0)}^{\mu \nu}(x)\right| 0\right\rangle \\
& -4 \eta^{\mu_{1} v_{1}} \delta\left(x-x_{1}\right)\left\langle 0\left|\frac{\delta^{2} S}{\delta h_{\mu \nu}(x) \delta h_{\mu_{2} \nu_{2}}\left(x_{2}\right)}\right| 0\right\rangle \\
& -4 \eta^{\mu_{2} \nu_{2}} \delta\left(x-x_{2}\right)\left\langle 0\left|\frac{\delta^{2} S}{\delta h_{\mu \nu}(x) \delta h_{\mu_{1} \nu_{1}}\left(x_{1}\right)}\right| 0\right\rangle \\
& +8\left\langle 0\left|\frac{\delta^{3} S}{\delta h_{\mu \nu}(x) \delta h_{\mu_{1} v_{1}}\left(x_{1}\right) h_{\mu_{2} \nu_{2}}\left(x_{2}\right)}\right| 0\right\rangle \text {. }
\end{aligned}
$$


The functional derivatives of $S$ with respect to $h$ are understood to be evaluated at $h=0$.

In the sequel we will need the explicit expressions of vertices, up to order two in $h$ (for a derivation of Feynman rules see Appendix B, in particular B.1 and B.2). Beside (32) and (33) we have

$$
\begin{aligned}
& \left.V_{f f h}^{\prime}: \quad \frac{i}{4} \eta_{\mu v}(\not p+\not p)^{\prime}\right) P_{L}, \\
& V_{f f h h}^{\prime}: \frac{3 i}{64}\left[\left(\left(p+p^{\prime}\right)_{\mu} \gamma_{\mu^{\prime}} \eta_{\nu v^{\prime}}+\left(p+p^{\prime}\right)_{\mu} \gamma_{\nu^{\prime}} \eta_{\nu \mu^{\prime}}\right.\right. \\
& +\{\mu \leftrightarrow v\})+\left(\left(p+p^{\prime}\right)_{\mu^{\prime}} \gamma_{\mu} \eta_{v v^{\prime}}\right. \\
& \left.\left.+\left(p+p^{\prime}\right)_{\mu^{\prime}} \gamma_{\nu} \eta_{\mu v^{\prime}}+\left\{\mu^{\prime} \leftrightarrow \nu^{\prime}\right\}\right)\right] P_{L}, \\
& V_{f f h h}^{\prime \prime}:-\frac{i}{16}\left[\eta_{\mu \nu}\left(\left(p+p^{\prime}\right)_{\mu^{\prime}} \gamma_{\nu^{\prime}}+\left(p+p^{\prime}\right)_{v^{\prime}} \gamma_{\mu^{\prime}}\right)\right. \\
& \left.+\eta_{\mu^{\prime} \nu^{\prime}}\left(\left(p+p^{\prime}\right)_{\mu} \gamma_{\nu}+\left(p+p^{\prime}\right)_{\nu} \gamma_{\mu}\right)\right] P_{L}, \\
& V_{f f h h}^{\prime \prime \prime}: \frac{i}{8}\left(\not p+\not p^{\prime}\right)\left(\eta_{\mu \nu} \eta_{\mu^{\prime} v^{\prime}}-\eta_{\mu \nu^{\prime}} \eta_{\mu^{\prime} v}-\eta_{\mu \mu^{\prime}} \eta_{\nu v^{\prime}}\right) P_{L}
\end{aligned}
$$

So far we have been completely general. From now on we consider only odd correlators, that is, only correlators linear in $\epsilon_{\mu \nu \lambda \rho}$. To start with, to $\left\langle 0\left|T_{(0) \mu}{ }^{\mu}(x)\right| 0\right\rangle$, which is a constant, only a tadpole can contribute, but its odd part vanishes because there is no scalar one can construct with $\epsilon$ and $\eta$. For the same reason also $\left\langle 0\left|T_{(0)}^{\mu \nu}(x)\right| 0\right\rangle$ vanishes. The twopoint function $\left\langle 0\left|\mathcal{T} T_{(0)}^{\mu v}(x) T_{(0)}^{\mu_{1} \nu_{1}}\left(x_{1}\right)\right| 0\right\rangle$ also must vanish, because in momentum space it must be a 4-tensor linear in $\epsilon$ and formed with $\eta$ and the momentum $k$ : there is no such tensor, symmetric in $\mu \leftrightarrow v, \mu_{1} \leftrightarrow v_{1}$ and $(\mu, v) \leftrightarrow\left(\mu_{1}, v_{1}\right)$. As for the terms $\left\langle 0\left|\frac{\delta^{2} S}{\delta h_{\mu \nu}(x) \delta h_{\mu_{1} \nu_{1}\left(x_{1}\right)}}\right| 0\right\rangle$ they might also produce non-vanishing contribution from tadpoles diagram, but like in the previous case it is impossible to satisfy the combinatorics. In conclusion (41) and (42) are identically satisfied, while (43) becomes

$$
\begin{aligned}
& \mathcal{T}^{(2)}(x)=\mathcal{T}_{\mu}^{\mu \mu_{1} v_{1} \mu_{2} v_{2}}\left(x, x_{1}, x_{2}\right) \\
& =\eta_{\mu \nu}\left(-\left\langle 0\left|\mathcal{T} T_{(0)}^{\mu \nu}(x) T_{(0)}^{\mu_{1} \nu_{1}}\left(x_{1}\right) T_{(0)}^{\mu_{2} \nu_{2}}\left(x_{2}\right)\right| 0\right\rangle\right. \\
& +4 i\left\langle 0\left|\mathcal{T} T_{(0)}^{\mu \nu}(x) \frac{\delta^{2} S}{\delta h_{\mu_{1} \nu_{1}}\left(x_{1}\right) \delta h_{\mu_{2} \nu_{2}}\left(x_{2}\right)}\right| 0\right\rangle \\
& +4 i\left\langle 0\left|\mathcal{T} T_{(0)}^{\mu_{1} v_{1}}\left(x_{1}\right) \frac{\delta^{2} S}{\delta h_{\mu \nu}(x) \delta h_{\mu_{2} \nu_{2}}\left(x_{2}\right)}\right| 0\right\rangle \\
& +4 i\left\langle 0\left|\mathcal{T} T_{(0)}^{\mu_{2} v_{2}}\left(x_{2}\right) \frac{\delta^{2} S}{\delta h_{\mu_{1} \nu_{1}}\left(x_{1}\right) \delta h_{\mu \nu}(x)}\right| 0\right\rangle \\
& \left.+8\left\langle 0\left|\frac{\delta^{3} S}{\delta h_{\mu \nu}(x) \delta h_{\mu_{1} \nu_{1}}\left(x_{1}\right) h_{\mu_{2} v_{2}}\left(x_{2}\right)}\right| 0\right\rangle\right) .
\end{aligned}
$$

To proceed we focus now on the terms containing the second derivative of $S$. Looking at (38) we see that there are several such terms. We argue now that those among them that do not contain the $\epsilon$ tensor, although the gamma trace algebra may generate an $\epsilon$ tensor, cannot contribute to the odd trace anomaly. The vertices corresponding to such terms have two fermion and two graviton legs, that is, they are of the type $V_{f f h h}$. By Fourier transform, we associate an incoming $e^{i p x}$ plane wave to one fermion and an outgoing $e^{-i p^{\prime} x}$ one to the other, while we associate two incoming plane waves $e^{i k_{1} x}, e^{i k_{2} x}$ to the two gravitons. Since none of them contain derivatives of $h$, the vertex will depend at most on $q=k_{1}+k_{2}$, not on $k_{1}-k_{2}$; see for instance the vertex coming from the third term in the first line of (38), i.e. $V_{f f h h}^{\prime}$.

This being so, the contributions from the terms related to the second derivative of $S$ in (51) via such vertices, and linear in $\epsilon$, must vanish, because it is impossible to form a 4-tensor symmetric in $\mu_{1} \leftrightarrow v_{1}, \mu_{2} \leftrightarrow v_{2}$ and $\left(\mu_{1}, v_{1}\right) \leftrightarrow\left(\mu_{2} v_{2}\right)$ with $\epsilon, \eta$ and $q_{\mu}$. It follows that only the contribution with the vertex $V_{f f h h}^{\epsilon}$ might contribute non-trivially to the odd trace anomaly. Looking at the form of $V_{f f h h}^{\epsilon}$, it is clear that the two terms in the third line of (51) give vanishing contribution because the contraction of $\mu$ with $v$ becomes a (vanishing) contraction of the $t$ tensor, (34).

Next let us consider the fourth line of (51). These are seagull terms, with three external graviton lines attached to the same point of a fermion loop. The gamma trace algebra cannot generate an $\epsilon$ tensor from all such terms, except of course the second term in the second line and the one in the fourth line. Therefore we can exclude all the former from our consideration. As for the latter the relevant vertex has two fermion legs, with the usual momenta $p$ and $p^{\prime}$, and three graviton legs, with incoming momenta $k_{1}, k_{2}, k_{3}$ and labels $\mu_{1}, \nu_{1}, \mu_{2}, \nu_{2}$ and $\mu_{3}, \nu_{3}$, respectively. Its expression for the second term in the second line of (51) is

$$
\sim \epsilon_{\mu_{2} \mu_{3} \lambda \rho} k_{3}^{\lambda} \gamma^{\rho} \eta_{\mu_{1} v_{3}} \eta_{\nu_{1} \nu_{2}}
$$

symmetrized in $\mu_{1} \leftrightarrow v_{1}, \mu_{2} \leftrightarrow v_{2}, \mu_{3} \leftrightarrow v_{3}$, and with respect to the exchange of any two couples $\left(\mu_{i}, v_{i}\right)$. The seagull term is therefore proportional to

$$
\int \mathrm{d}^{4} p \frac{p^{\rho}}{p^{2}}
$$

which vanishes. As for the term in the fourth line of (51), one comes to similar conclusions.

In summary, the odd trace anomaly receives contributions only from

$$
\begin{aligned}
\mathcal{T}^{(2)}(x)= & \mathcal{T}_{\mu}^{\mu \mu_{1} v_{1} \mu_{2} \nu_{2}}\left(x, x_{1}, x_{2}\right) \\
= & \eta_{\mu \nu}\left(-\left\langle 0\left|\mathcal{T} T_{(0)}^{\mu v}(x) T_{(0)}^{\mu_{1} \nu_{1}}\left(x_{1}\right) T_{(0)}^{\mu_{2} v_{2}}\left(x_{2}\right)\right| 0\right\rangle\right. \\
& \left.+4 i\left\langle 0\left|\mathcal{T} T_{(0)}^{\mu \nu}(x) \frac{\delta^{2} S}{\delta h_{\mu_{1} \nu_{1}}\left(x_{1}\right) \delta h_{\mu_{2} \nu_{2}}\left(x_{2}\right)}\right| 0\right\rangle\right) .
\end{aligned}
$$

This result looks very much like the starting point of [1], i.e. it seems to reduce to the same contributions, i.e. the triangle 
diagram and bubble diagram (which turned out to vanish), but there is an important modification: the $T_{(0)}^{\mu \nu}(x)$ is different from the free e.m. tensor in [1], the definition (44) contains an additional piece (the second). It is not hard to show that the second term in the RHS of (53) vanishes also when taking account of this modification. As for the threepoint function in the first term of (53) we obtain of course the same result as in [1] when the calculation is made with three vertices $V_{f f h}: P-V_{f f h}-P-V_{f f h}-P-V_{f f h}$ (for the reader's convenience this calculation is repeated in Appendix A); it is 0 when the second or third vertices are replaced by $V_{f f h}^{\prime}$, and it is -4 times the result of [1] if the first vertex is replaced by $V_{f f h}^{\prime}$, i.e. $P-V_{f f h}^{\prime}-P-V_{f f h}-P-V_{f f h}$. When we replace more than one vertex $V_{f f h}$ with $V_{f f h}^{\prime}$ we get 0 . So the overall result of (53) is $(1-4=-3)$ times the end result for the trace anomaly in [1].

We will see below, however, that this modification of the anomaly must be canceled in order to guarantee conservation. Let us call the lowest order integrated anomaly, obtained in [1], $\mathcal{A}_{\omega}=-\int \omega \mathcal{A}_{0}$. Then the new addition equals $-4 \mathcal{A}_{\omega}$. By adding to the effective action the term $\mathcal{C}=-\int \frac{1}{2} \operatorname{tr} h \mathcal{A}_{0}$ we exactly cancel this additional unwanted piece. We will verify that this counterterm cancels an analogous anomalous term in the Ward identity of the diffeomorphisms, anomalous term which is generated by the same diagram $P-V_{f f h}^{\prime}-P-$ $V_{f f h}-P-V_{f f h}$, which is the cause of the additional term in question in the trace anomaly.

In conclusion, the only relevant term for the odd trace anomaly is the $P-V_{f f h}-P-V_{f f h}-P-V_{f f h}$ one. This is the term we have computed first in [1], which gives rise to the Pontryagin anomaly. It should be remarked that in the odd trace anomaly calculation there are no contributions from tadpole and seagull terms.

\subsection{Odd trace anomaly for Dirac and Majorana fermions}

The action for a Dirac fermion is the same as in (38) with $\psi_{L}$ everywhere replaced by the Dirac fermion $\psi$. In order to evaluate the odd trace anomaly we remark that an odd contribution in (46) can come only from the terms in (38) that contain the $\epsilon$ tensor. Since these terms contain $\gamma_{5}$, upon tracing the gamma matrix part, either they give 0 or another $\epsilon$ tensor. In the latter case they produce an even contribution to the trace anomaly, which does not concern us here. In conclusion the odd trace anomaly, in the case of a Dirac fermion, vanishes.

When the fermion are Majorana the conclusion does not change. The simplest way to see it is to use the Majorana representation for the gamma matrices. Then $\psi$ has four real components, and the only change with respect to the Dirac case is that in the path integral we integrate over real fermion fields instead of complex ones, while all the rest remains unchanged. The conclusion is obvious.

\section{Conservation of the e.m. tensor}

As already anticipated above, trace anomalies are strictly connected with diffeomorphism anomalies. In $4 d$ the socalled Einstein-Lorentz anomalies are absent, but there may appear other anomalous terms in the Ward identity of the diffeomorphisms. The latter together with a Weyl anomaly partner form a cocycle of the joint diff + Weyl cohomology; see $[19,20]$. Usually, by adding a local counterterm to the effective action, one can restore diffeomorphism invariance. In the present case, odd parity trace anomaly, the analysis of such possible anomalies was carried out in a simplified form in [2]. In this section we wish to complete that analysis by considering also tadpoles and seagull terms.

If we take into account the tadpole and seagull terms in the conservation law one has to take into account also the VEV of the e.m. tensor. Let us set

$\left\langle 0\left|T_{(0)}^{\mu \nu}(x)\right| 0\right\rangle=\left\langle 0\left|T_{(0)}^{\mu \nu}(0)\right| 0\right\rangle=\Theta^{\mu \nu}=A \eta^{\mu \nu}$.

The Ward identity is

$$
\begin{aligned}
\nabla_{\mu}\left\langle\left\langle T^{\mu \nu}(x)\right\rangle\right\rangle= & \partial_{\mu}\left\langle\left\langle T^{\mu v}(x)\right\rangle\right\rangle+\Gamma_{\mu \lambda}^{\mu}\left\langle\left\langle T^{\lambda v}(x)\right\rangle\right\rangle \\
& +\Gamma_{\mu \lambda}^{v}\left\langle\left\langle T^{\mu \lambda}(x)\right\rangle\right\rangle=0
\end{aligned}
$$

because $\left\langle\left\langle T^{\mu \nu}(x)\right\rangle\right\rangle \equiv \frac{2}{\sqrt{-g}} \frac{\delta W}{\delta g_{\mu \nu}(x)}$. To first order in $h_{\mu \nu}$ we have

$$
\begin{aligned}
& \Gamma_{\mu \lambda}^{v}(x) \approx \frac{1}{2}\left(\partial_{\mu} h_{\lambda}^{v}+\partial_{\lambda} h_{\mu}^{\nu}-\partial^{\nu} h_{\mu \lambda}\right) \\
& \Gamma_{\mu \lambda}^{\mu}(x) \approx \frac{1}{2} \partial_{\lambda} h_{\mu}^{\mu} .
\end{aligned}
$$

Now we use (40), (44), (45) and (46). To the zeroth order in $h$ (55) implies

$\partial_{\mu}\left\langle 0\left|T^{\mu \nu}(x)\right| 0\right\rangle=0$.

To get the WI to first order one must differentiate (55) with respect to $h_{\mu \nu}$. One has

$\frac{\delta h_{\mu \nu}(x)}{\delta h_{\lambda \rho}(y)}=\frac{1}{2}\left(\delta_{\mu}^{\lambda} \delta_{v}^{\rho}+\delta_{v}^{\lambda} \delta_{\mu}^{\rho}\right) \delta(x-y)$

Differentiating the first term on the RHS of (55) one gets the ordinary divergence of the two-point function. Then

$\frac{\delta \Gamma_{\mu \lambda}^{\mu}(x)}{\delta h_{\mu_{1} v_{1}}(y)}=\frac{1}{2} \eta^{\mu_{1} v_{1}} \partial_{\lambda}^{x} \delta(x-y)$ 
and

$$
\begin{aligned}
\frac{\delta \Gamma_{\mu \lambda}^{\nu}(x)}{\delta h_{\mu_{1} \nu_{1}}(y)}= & \frac{1}{4}\left(\partial_{\mu} \delta(x-y)\left(\delta_{\lambda}^{\nu_{1}} \eta^{\mu_{1} v}+\delta_{\lambda}^{\mu_{1}} \eta^{\nu_{1} v}\right)\right. \\
& +\partial_{\lambda} \delta(x-y)\left(\delta_{\mu}^{\mu_{1}} \eta^{\nu \nu_{1}}+\delta_{\mu}^{\nu_{1}} \eta^{\nu \mu_{1}}\right) \\
& \left.-\partial^{v} \delta(x-y)\left(\delta_{\lambda}^{\nu_{1}} \delta_{\mu}^{\mu_{1}}+\delta_{\lambda}^{\mu_{1}} \delta_{\mu}^{\nu_{1}}\right)\right)
\end{aligned}
$$

Putting everything together one finds

$$
\begin{aligned}
\partial_{\mu}^{x} \mathcal{T}^{\mu v \mu_{1} v_{1}}(x, y)+\frac{1}{2} \eta^{\mu_{1} v_{1}} \partial_{\lambda}^{x} \delta(x-y) \Theta^{\lambda v} \\
\quad+\frac{1}{2}\left(\partial_{\lambda}^{x} \delta(x-y) \eta^{\mu_{1} v} \Theta^{\lambda v_{1}}\right. \\
\left.\quad+\partial_{\lambda}^{x} \delta(x-y) \eta^{\nu_{1} v} \Theta^{\lambda \mu_{1}}-\partial^{x v} \delta(x-y) \Theta^{\mu_{1} v_{1}}\right) \\
=i \partial_{\mu}^{x}\left\langle 0\left|\mathcal{T} T_{(0)}^{\mu v}(x) T_{(0)}^{\mu_{1} v_{1}}(y)\right|\right\rangle \\
\quad+4 \partial_{\mu}^{x}\left\langle 0\left|\frac{\delta^{2} S}{\delta h_{\mu \nu}(x) \delta h_{\mu_{1} v_{1}}(y)}\right| 0\right\rangle \\
\quad+\partial_{\lambda}^{x} \delta(x-y) \eta^{\mu_{1} v} \Theta^{\lambda v_{1}} \\
\quad+\partial_{\lambda}^{x} \delta(x-y) \eta^{\nu_{1} v} \Theta^{\lambda \mu_{1}}-\partial^{x v} \delta(x-y) \Theta^{\mu_{1} v_{1}}=0 .
\end{aligned}
$$

We have already noted that, for what concerns the odd part, all the terms in the RHS vanish. Therefore conservation is guaranteed up to second order in $h$.

The order three Ward identity has a rather cumbersome expression, in particular it contains various terms linear in $\Theta^{\mu \nu}$. Since they do not contribute to the odd part of the identity we drop them altogether. The remaining terms are

$$
\begin{aligned}
& -\partial_{\mu}^{x}\left\langle 0\left|\mathcal{T} T_{(0)}^{\mu v}(x) T_{(0)}^{\mu_{1} \nu_{1}}\left(x_{1}\right) T_{(0)}^{\mu_{2} \nu_{2}}\left(x_{2}\right)\right| 0\right\rangle \\
& +4 i \partial_{\mu}^{x}\left\langle 0\left|\mathcal{T} T_{(0)}^{\mu v}(x) \frac{\delta^{2} S}{\delta h_{\mu_{1} \nu_{1}}\left(x_{1}\right) \delta h_{\mu_{2} \nu_{2}}\left(x_{2}\right)}\right| 0\right\rangle \\
& +4 i \partial_{\mu}^{x}\left\langle 0\left|\mathcal{T} T_{(0)}^{\mu_{2} v_{2}}\left(x_{2}\right) \frac{\delta^{2} S}{\delta h_{\mu v}(x) \delta h_{\mu_{1} v_{1}}\left(x_{1}\right)}\right| 0\right\rangle \\
& +4 i \partial_{\mu}^{x}\left\langle 0\left|\mathcal{T} T_{(0)}^{\mu_{1} v_{1}}\left(x_{1}\right) \frac{\delta^{2} S}{\delta h_{\mu \nu}(x) \delta h_{\mu_{2} v_{2}}\left(x_{2}\right)}\right| 0\right\rangle \\
& -4 \eta^{\mu_{1} v_{1}} \partial_{\mu}^{x}\left(\delta\left(x-x_{1}\right)\left\langle 0\left|\frac{\delta^{2} S}{\delta h_{\mu \nu}(x) \delta h_{\mu_{2} v_{2}}\left(x_{2}\right)}\right| 0\right\rangle\right) \\
& -4 \eta^{\mu_{2} v_{2}} \partial_{\mu}^{x}\left(\delta\left(x-x_{2}\right)\left\langle 0\left|\frac{\delta^{2} S}{\delta h_{\mu \nu}(x) \delta h_{\mu_{1} v_{1}}\left(x_{1}\right)}\right| 0\right\rangle\right) \\
& -i \partial_{\mu}^{x}\left(\delta\left(x-x_{1}\right) \eta^{\mu_{1} \nu_{1}}\left\langle 0 \mid \mathcal{T} T_{(0)}^{\mu v}(x) T_{(0)}^{\mu_{2} v_{2}}\left(x_{2}\right)\right\rangle\right. \\
& \left.+\delta\left(x-x_{2}\right) \eta^{\mu_{2} v_{2}}\left\langle 0 \mid \mathcal{T} T_{(0)}^{\mu v}(x) T_{(0)}^{\mu_{1} v_{1}}\left(x_{1}\right)\right\rangle\right) \\
& +\partial_{\lambda}^{x} \delta\left(x-x_{1}\right) \eta^{\mu_{1} \nu_{1}}\left(i\left\langle 0\left|\mathcal{T} T_{(0)}^{\lambda v}(x) T_{(0)}^{\mu_{2} \nu_{2}}\left(x_{2}\right)\right| 0\right\rangle\right. \\
& \left.+4\left\langle 0\left|\frac{\delta^{2} S}{\delta h_{\lambda v}(x) \delta h_{\mu_{2} v_{2}}\left(x_{2}\right)}\right| 0\right\rangle\right) \\
& +\partial_{\lambda}^{x} \delta\left(x-x_{2}\right) \eta^{\mu_{2} v_{2}}\left(i\left\langle 0\left|\mathcal{T} T_{(0)}^{\lambda \nu}(x) T_{(0)}^{\mu_{1} \nu_{1}}\left(x_{1}\right)\right| 0\right\rangle\right. \\
& \left.+4\left\langle 0\left|\frac{\delta^{2} S}{\delta h_{\lambda v}(x) \delta h_{\mu_{1} v_{1}}\left(x_{1}\right)}\right| 0\right\rangle\right) \\
& +\left(\partial_{\mu}^{x} \delta\left(x-x_{1}\right)\left(\delta_{\lambda}^{\mu_{1}} \eta^{\nu \nu_{1}}+\delta_{\lambda}^{\nu_{1}} \eta^{\nu \mu_{1}}\right)-\partial^{x v} \delta\left(x-x_{1}\right) \delta_{\mu}^{\mu_{1}} \delta_{\lambda}^{\nu_{1}}\right)
\end{aligned}
$$

$$
\begin{aligned}
& \cdot\left(i\left\langle 0 \mid \mathcal{T} T_{(0)}^{\mu \lambda}(x) T_{(0)}^{\mu_{2} v_{2}}\left(x_{2}\right)\right\rangle+4\left\langle 0\left|\frac{\delta^{2} S}{\delta h_{\mu \lambda}(x) \delta h_{\mu_{2} \nu_{2}}\left(x_{2}\right)}\right| 0\right\rangle\right) \\
& +\left(\partial_{\mu}^{x} \delta\left(x-x_{2}\right)\left(\delta_{\lambda}^{\mu_{2}} \eta^{\nu \nu_{2}}+\delta_{\lambda}^{\nu_{2}} \eta^{v \mu_{2}}\right)-\partial^{x v} \delta\left(x-x_{2}\right) \delta_{\mu}^{\mu_{2}} \delta_{\lambda}^{\nu_{2}}\right) \\
& \cdot\left(i\left\langle 0 \mid \mathcal{T} T_{(0)}^{\mu \lambda}(x) T_{(0)}^{\mu_{2} \nu_{2}}\left(x_{2}\right)\right\rangle+4\left\langle 0\left|\frac{\delta^{2} S}{\delta h_{\mu \lambda}(x) \delta h_{\mu_{1} \nu_{1}}\left(x_{1}\right)}\right| 0\right\rangle\right) \\
& +8 \partial_{\mu}^{x}\left\langle 0\left|\frac{\delta^{3} S}{\delta h_{\lambda v}(x) \delta h_{\mu_{1} \nu_{1}}\left(x_{1}\right) \delta h_{\mu_{2} v_{2}}\left(x_{2}\right)}\right| 0\right\rangle=0 .
\end{aligned}
$$

In the above discussion concerning the odd trace anomaly we have already met some of the terms appearing in this formula. As already noted there, the two-point functions $\left\langle 0\left|\mathcal{T} T_{(0)}^{\mu \nu}(x) T_{(0)}^{\lambda \rho}(y)\right| 0\right\rangle$ cannot contribute to the odd part because the combinatorics of the $\epsilon$ and $\eta$ tensor plus an external momentum does not allow it. Next the VEV's of second and third derivative of $S$ with respect to $h$ cannot contribute with a tadpole term: if we look at (38) and focus on the vertices that can give an odd parity contribution, i.e. those containing the $\epsilon$ tensor, we notice that they depend linearly on the external momenta (not on the fermion momenta); therefore, in a tadpole term, the momentum integrand can only be linear in the internal momentum $p^{\mu}$, and thus vanishes.

Therefore (62), as far as the odd part is concerned, reduces to

$$
\begin{aligned}
& -\partial_{\mu}^{x}\left\langle 0\left|\mathcal{T} T_{(0)}^{\mu v}(x) T_{(0)}^{\mu_{1} \nu_{1}}\left(x_{1}\right) T_{(0)}^{\mu_{2} v_{2}}\left(x_{2}\right)\right| 0\right\rangle \\
& +4 i \partial_{\mu}^{x}\left\langle 0\left|\mathcal{T} T_{(0)}^{\mu v}(x) \frac{\delta^{2} S}{\delta h_{\mu_{1} v_{1}}\left(x_{1}\right) \delta h_{\mu_{2} v_{2}}\left(x_{2}\right)}\right| 0\right\rangle \\
& +4 i \partial_{\mu}^{x}\left\langle 0\left|\mathcal{T} T_{(0)}^{\mu_{2} v_{2}}\left(x_{2}\right) \frac{\delta^{2} S}{\delta h_{\mu v}(x) \delta h_{\mu_{1} v_{1}}\left(x_{1}\right)}\right| 0\right\rangle \\
& +4 i \partial_{\mu}^{x}\left\langle 0\left|\mathcal{T} T_{(0)}^{\mu_{1} \nu_{1}}\left(x_{1}\right) \frac{\delta^{2} S}{\delta h_{\mu v}(x) \delta h_{\mu_{2} v_{2}}\left(x_{2}\right)}\right| 0\right\rangle=0 .
\end{aligned}
$$

The last three terms on the LHS can be shown to vanish. The proof is not as simple as the previous ones. One has to push the calculations one step further, introduce a dimensional regulator and use Feynman parametrization (see Appendix B). The integration over the relevant parameter can easily be shown to vanish. What remains to be verified is therefore

$\partial_{\mu}^{x}\left\langle 0\left|\mathcal{T} T_{(0)}^{\mu \nu}(x) T_{(0)}^{\mu_{1} \nu_{1}}\left(x_{1}\right) T_{(0)}^{\mu_{2} v_{2}}\left(x_{2}\right)\right| 0\right\rangle=0$.

Let us consider the term generated by the diagram $P-V_{f f h^{-}}^{\prime}$ $P-V_{f f h}-P-V_{f f h}$. We have already calculated it above, it equals $-\partial_{v}^{x} \mathcal{A}(x)$, where $\mathcal{A}(x)$ is the unintegrated Weyl anomaly calculated in [1]. So conservation is violated by this term. Adding to the action the term $\mathcal{C}=-\int \frac{1}{2} \operatorname{tr} h \omega \mathcal{A}_{0}$, as we have anticipated above, we get the diff variation

$\delta_{\xi} \mathcal{C}=-\int \partial_{\nu} \xi^{\nu} \mathcal{A}=\int \xi^{\nu} \partial_{\nu} \mathcal{A}$,

which exactly cancels this anomaly. ${ }^{6}$

\footnotetext{
${ }^{6}$ Concerning the signs remember that there is a relative - sign between the unintegrated Diff and trace anomalies.
} 
Next we have to consider the diagram $P-V_{f f h}-P-V_{f f h}^{\prime}-$ $P-V_{f f h}$ and $P-V_{f f h}-P-V_{f f h}-P-V_{f f h}^{\prime}$. In the on-shell case, $k_{1}^{2}=0=k_{2}^{2}$, these contributions can be shown to vanish. It is enough to take Eq. (3.18) of [1]. The first diagram corresponds to contracting this formula with $k_{1}^{\mu}$ or $k_{1}^{v}$. It is easy to see that such a contraction vanishes. The second diagram corresponds to contracting the same formula with $k_{2}^{\mu^{\prime}}$ or $k_{2}^{\nu^{\prime}}$, which again vanishes. Therefore, at least in the on-shell case these diagrams do not contribute.

In conclusion we have to verify (64) for the triangle diagram $P-V_{f f h}-P-V_{f f h}-P-V_{f f h}$ (and the crossed one). This is what we have already done in $[1,2]$.

\subsection{On-shell, off-shell and locality}

In $[1,2]$ the following integrals were used in order to compute the relevant Feynman diagram:

$$
\begin{aligned}
& \int \frac{\mathrm{d}^{4} p}{(2 \pi)^{4}} \int \frac{d^{\delta} \ell}{(2 \pi)^{\delta}} \frac{p^{2}}{\left(p^{2}+\ell^{2}+\Delta\right)^{3}} \\
& =\frac{1}{(4 \pi)^{2}}\left(-\frac{2}{\delta}-\gamma+\log (4 \pi)-\log \Delta\right) \\
& \int \frac{\mathrm{d}^{4} p}{(2 \pi)^{4}} \int \frac{d^{\delta} \ell}{(2 \pi)^{\delta}} \frac{p^{4}}{\left(p^{2}+\ell^{2}+\Delta\right)^{3}} \\
& =\frac{\Delta}{2(4 \pi)^{2}}\left(-\frac{2}{\delta}-\gamma+4+\log (4 \pi)-\log \Delta\right)
\end{aligned}
$$

and

$$
\begin{aligned}
& \int \frac{\mathrm{d}^{4} p}{(2 \pi)^{4}} \int \frac{d^{\delta} \ell}{(2 \pi)^{\delta}} \frac{\ell^{2}}{\left(p^{2}+\ell^{2}+\Delta\right)^{3}}=-\frac{1}{2(4 \pi)^{2}} \\
& \int \frac{\mathrm{d}^{4} p}{(2 \pi)^{4}} \int \frac{d^{\delta} \ell}{(2 \pi)^{\delta}} \frac{\ell^{2} p^{2}}{\left(p^{2}+\ell^{2}+\Delta\right)^{3}}=\frac{1}{(4 \pi)^{2}} \Delta
\end{aligned}
$$

where $\Delta=u(1-u) k_{1}^{2}+v(1-v) k_{2}^{2}+2 u v k_{1} k_{2}, u, v$ are Feynman parameters, and $\delta$ is the dimensional regulator: $d=$ $4+\delta$.

The odd trace anomaly is due to the term $[1,2]$

$$
\begin{aligned}
- & \frac{1}{128} \int \frac{\mathrm{d}^{4} p}{(2 \pi)^{4}} \int \frac{d^{\delta} \ell}{(2 \pi)^{\delta}} \operatorname{tr}\left(\frac{\not p+\ell}{p^{2}-\ell^{2}}\left(2 p-k_{1}\right)_{\lambda} \gamma_{\rho}\right. \\
\times & \left.\frac{\not p+\ell-\not k_{1}}{\left(p-k_{1}\right)^{2}-\ell^{2}}\left(2 p-2 k_{1}-k_{2}\right)_{\alpha} \gamma_{\beta} \frac{\not p+\ell-q}{(p-q)^{2}-\ell^{2}} \ell \frac{\gamma_{5}}{2}\right) .
\end{aligned}
$$

This requires the two integrals (67), which must be further integrated on $v$ from 0 to $1-u$ and on $u$ from 0 to 1 . The integrations over the Feynman parameters are elementary and lead to the result

$$
\begin{aligned}
\mathcal{T}_{\mu \alpha \beta \lambda \rho}^{\mu}\left(k_{1}, k_{2}\right)= & \frac{1}{192(4 \pi)^{2}} k_{1}^{\sigma} k_{2}^{\tau}\left(t _ { \lambda \rho \alpha \beta \sigma \tau } \left(k_{1}^{2}\right.\right. \\
& \left.\left.+k_{2}^{2}+k_{1} k_{2}\right)-t_{\lambda \rho \alpha \beta \sigma \tau}^{(21)}\right) .
\end{aligned}
$$

We report this result here to stress the fact that the terms contained in it are contact terms and thus lead to a local anomaly. In [2] we remarked that the piece proportional to $\left(k_{1}^{2}+k_{2}^{2}\right)$ disappears on shell, and off-shell corresponds to a trivial anomaly.

To compute the conservation law (64) we need also the integrals (66). It is evident from the form of their RHS's that integrating on $u$ and $v$ will lead to non-contact terms, and nonlocal expressions for the odd diff anomaly. However, if we put $k_{1}$ and $k_{2}$ on shell things change. The contact terms have been discussed in [2]. They can be eliminated by subtracting local counterterms without spoiling the trace anomaly. As for the non-contact terms they are polynomials of $k_{1}$ and $k_{2}$ multiplied by $\log k_{1} \cdot k_{2}$. All such terms are listed in Appendix E of [2]. They look non-local. However, using the Fourier transform

$$
\begin{aligned}
& \int \frac{\mathrm{d}^{4} k_{1}}{(2 \pi)^{4}} \frac{\mathrm{d}^{4} k_{2}}{(2 \pi)^{4}} e^{i\left(k_{1}(x-z)+k_{2}(y-z)\right)} \log \left(k_{1}+k_{2}\right)^{2} \\
& =-\frac{1}{4 \pi^{2}} \delta^{(4)}(x-y) \square_{z}\left(\frac{1}{(x-z)^{2}} \log \frac{(x-z)^{2}}{4}\right),
\end{aligned}
$$

one can show that they give a vanishing contribution when inserted into the effective action, because of the on-shell condition $\square h_{\mu \nu}=0$ (De Donder gauge). On the other hand, when $k_{1}$ and $k_{2}$ are off shell, the anomaly looks non-local. This is a surprise because we are used to think of anomalies as local expressions. But we have learned from [21] and from the higher spins analysis that when higher spins are involved (including the metric) covariance generally requires one to sacrifice locality. However, the ensuing non-locality is a gauge artifact. By imposing a suitable gauge choice, locality can be restored. As an example see Eq. (8.21) and others in [21].

\section{Additional remarks on Weyl and Majorana}

Before leaving Part I of this paper let us add some comments on the Pontryagin trace anomaly. A non-trivial property is that it belongs to the family of chiral anomalies characterized by having opposite coefficients for opposite chiralities. ${ }^{7}$ This anomaly did not appear for the first time in [1]. The possibility of its existence due to its Wess-Zumino consistency was pointed out in [22] and, although somewhat implicitly, its

\footnotetext{
7 This family includes in particular the consistent chiral anomalies in gauge theories. Thus in selecting the regularization to compute the odd trace anomaly, a necessary criterion is the ability to reproduce such well-known consistent gauge anomalies.
} 
existence was implied by [23]. A similar anomaly was found in a different contest (originating from an antisymmetric tensor field) in the framework of an AdS/CFT in [24], where a possible conflict with unitarity was pointed out. The same risk has been pointed out, from a different viewpoint, in the introduction of the present paper and in [1]. In general it seems that its presence signals some kind of difficulty in properly defining the theory. Very likely for this reason the existence of the Pontryagin trace anomaly for chiral fermions is still considered controversial and objections have been raised against it. Such objections are often reducible to the credence that Weyl fermions are equivalent to massless Majorana fermions. We have already answered this naive objection and will not come back to it. There are more serious issues however. One is the following. In conformal field theory in $4 \mathrm{~d}$ the three-point functions of the energy-momentum tensor cannot have an odd part, so how can an anomaly arise from the regularization of a vanishing bare correlator? We have already answered this question in [2]: an anomaly can arise as a simple quantum effect; we have shown other examples of correlators which do not arise from the regularization of non-vanishing bare correlators, [25]. The crucial criterion is consistency.

A frequent prejudice is based on the lore that anomalies appear only in connection with complex representations of the gauge group in question. This is actually true in many cases for consistent chiral gauge anomalies. The latter are linear in the completely symmetric ad-invariant tensors of order $n$ in even $d=2 n-2$ dimension. For instance in $d=4$ the tensor in question is the symmetric tensor $d^{a b c}=\frac{1}{2} \operatorname{tr}\left(T^{a}\left\{T^{b}, T^{c}\right\}\right)$ with $T^{a}$ being the anti-hermitean generators of the Lie algebra. It is clear that if the representation is real, i.e. $T^{a}$ is antisymmetric, $d^{a b c}$ vanishes. For instance in $4 \mathrm{~d}$ there are no Einstein-Lorentz (a.k.a. diffeomorphisms and/or local Lorentz anomalies), because the corresponding representation of the Lorentz group is real. However, one cannot blindly tranfer the above criterion to the case of trace anomalies. A hint that in such a case it may not apply is the following: as we have explained in Sect. 2, the fermionic functional determinant in a left-handed theory can be thought of as a square root. This square root is likely to give rise to a phase, which in turn would explain the imaginary anomaly. To the best of our knowledge, it is impossible to decide this a priori. Therefore we can only rely on the explicit computation.

Another objection may arise from the following consideration. Let us split the Dirac fermion into two Weyl fermions: $\psi=P_{L} \psi+P_{R} \hat{\psi}=\psi_{L}+\psi_{R}$. The terms that appear in (38), in the Dirac case, are of the form $\bar{\psi} \gamma^{a} \stackrel{\leftrightarrow}{\partial}_{m} \psi$ and $\bar{\psi} \gamma_{c} \gamma_{5} \psi$. They both split into the sum of the left and right-handed part. With simple manipulations we have

$\bar{\psi}_{R} \gamma^{a} \stackrel{\leftrightarrow}{\partial}_{m} \psi_{R}=\psi^{\dagger} \gamma^{0} \gamma^{a} P_{R} \stackrel{\leftrightarrow}{\partial}_{m} \psi$

$$
\begin{aligned}
& =\psi^{T} C^{-1} \gamma^{a} \gamma^{0} P_{R} C \stackrel{\leftrightarrow}{\partial}_{m} \psi^{*} \\
& =\overline{\hat{\psi}} P_{R} \gamma^{a} P_{L} \overleftrightarrow{\partial}_{m} \hat{\psi}=\overline{\hat{\psi}_{L}} \gamma^{a} \stackrel{\leftrightarrow}{\partial}_{m} \hat{\psi}_{L}
\end{aligned}
$$

where $\hat{\psi}=\gamma^{0} C \psi^{*}$, and a similar expression for $\bar{\psi} \gamma_{c} \gamma_{5} \psi$. Thus, for instance, we can write

$\bar{\psi} \gamma^{a} \stackrel{\leftrightarrow}{\partial}_{m} \psi=\bar{\psi}_{L} \gamma^{a} \stackrel{\leftrightarrow}{\partial}_{m} \psi_{L}+\overline{\hat{\psi}}_{L} \gamma^{a} \stackrel{\leftrightarrow}{\partial}_{m} \hat{\psi}_{L}$.

Recall that $\widehat{\psi_{L}}=\hat{\psi}_{R}$. A Majorana fermion satisfies the reality condition $\psi=\widehat{\psi}$, so we can split it, according to the chiralities, $\psi=P_{L} \psi+P_{R} \hat{\psi}$. Then, looking at (72), we have for instance

$\bar{\psi} \gamma^{a} \stackrel{\leftrightarrow}{\partial}_{m} \psi=2 \bar{\psi}_{L} \gamma^{a} \stackrel{\leftrightarrow}{\partial}_{m} \psi_{L}$.

It would seem that the full Majorana action can be expressed as twice the action for its left-handed part. Then one would be led to conclude that there is an odd trace anomaly also for a Majorana fermion. This is another possible pitfall induced by a careless use of formal manipulations. The answer is the same as in Sect. 2: one cannot consider the passage from $\psi$ to $\psi_{L}$ in (73) as an allowed field redefinition, because it changes the integration measure. Majorana and Weyl fermions have their own appropriate actions, which faithfully represent their properties; in each case one must refer to the appropriate action, in particular, for Majorana fermions one should avoid using the RHS of (73).

Finally there is one subtle issue that has been somehow understood so far. We have stressed above that the crucial ingredient in the calculation of anomalies is the functional integral measure. We have also explained the problems connected with the latter when chiral fermions are involved. In Sect. 3 we have employed a Feynman diagram technique, tacitly assuming that it reproduces the correct path integration measure. Although this must be the case, because the relevant Feynman diagrams (with chiral propagators and chiral vertices) are different from those for Dirac or Majorana fermions, it is fair to say that we do not have a direct proof of it. There is, however, a way to spell out any residual doubts concerning the path integration measure. It relies in the analog of the method used by Bardeen, [4]; for chiral gauge anomalies, see also [13]. In such an approach one employs Dirac fermions (and, consequently, the ordinary Dirac measure) and recovers the chiral fermion theory as a subcase, by taking a specific limit. To this approach the second part of the paper is devoted.

\section{Part II}

In this second part we consider another approach to the odd trace anomaly, similar to Bardeen's method to chiral gauge anomalies in gauge theories, $[4,12,13]$. The latter consists in introducing both a vector and an axial potential as external sources of a free Dirac fermion theories in $4 \mathrm{~d}$. The usual con- 
sistent and covariant anomalies are obtained as specific limits of this model. In order to transfer to gravity such a model we need a second metric, an axial metric, beside the usual one. We will call such a model metric-axial-tensor (MAT) gravity.

\section{Metric-axial-tensor gravity}

\subsection{Axial metric}

We use the symbols $g_{\mu \nu}, g^{\mu \nu}$ and $e_{\mu}^{a}, e_{a}^{\mu}$ in the usual sense of metric and vierbein and their inverses. Then we introduce the formal writing ${ }^{8}$

$G_{\mu \nu}=g_{\mu \nu}+\gamma_{5} f_{\mu \nu}$

where $f$ is a symmetric tensor. Their background values are $\eta_{\mu \nu}$ and 0 , respectively. So, to first order

$g_{\mu \nu}=\eta_{\mu \nu}+h_{\mu \nu}, \quad f_{\mu \nu}=k_{\mu \nu}$.

In matrix notation the inverse of $G, G^{-1}$, is defined by

$G^{-1}=\hat{g}+\gamma_{5} \hat{f}, \quad G^{-1} G=1, \quad \hat{G}^{\mu \lambda} G_{\lambda v}=\delta_{v}^{\mu}$,

which implies

$\hat{g} f+\hat{f} g=0, \quad \hat{g} g+\hat{f} f=1$.

That is,

$\hat{f}=-\hat{g} f g^{-1}, \quad \hat{g}=\left(g-f g^{-1} f\right)^{-1}$.

So

$\hat{g}=\left(1-g^{-1} f g^{-1} f\right)^{-1} g^{-1}$,

$\hat{f}=-\left(1-g^{-1} f g^{-1} f\right)^{-1} g^{-1} f g^{-1}$

Keeping up to second order terms:

$$
\begin{aligned}
& g^{\mu v}=\eta^{\mu \nu}-h^{\mu \nu}+h_{\lambda}^{\mu} h^{\lambda v}+\cdots, \\
& \hat{g}^{\mu v}=\eta^{\mu \nu}-h^{\mu \nu}+h_{\lambda}^{\mu} h^{\lambda v}+k_{\lambda}^{\mu} k^{\lambda \nu}+\cdots, \\
& \hat{f}^{\mu \nu}=-k^{\mu \nu}+h_{\lambda}^{\mu} k^{\lambda \nu}+k_{\lambda}^{\mu} h^{\lambda \nu}+\cdots .
\end{aligned}
$$

\subsection{MAT vierbein}

Likewise for the vierbein one writes

$E_{\mu}^{a}=e_{\mu}^{a}+\gamma_{5} c_{\mu}^{a}, \quad \hat{E}_{a}^{\mu}=\hat{e}_{a}^{\mu}+\gamma_{5} \hat{c}_{a}^{\mu}$.

This implies

$$
\eta_{a b}\left(e_{\mu}^{a} e_{\nu}^{b}+c_{\mu}^{a} c_{\nu}^{b}\right)=g_{\mu \nu}, \quad \eta_{a b}\left(e_{\mu}^{a} c_{\nu}^{b}+e_{\nu}^{a} c_{\mu}^{b}\right)=f_{\mu \nu} .
$$

\footnotetext{
8 We use at times the suggestive terminology axial-complex for an expression like $G_{\mu \nu}$, axial-real for $g_{\mu \nu}$ and axial-imaginary for $f_{\mu \nu}$. This alludes to a geometrical interpretation, which is, however, not necessary to expand on in this paper.
}

Moreover, from $\hat{E}_{a}^{\mu} E_{v}^{a}=\delta_{v}^{\mu}$,

$\hat{e}_{a}^{\mu} c_{v}^{a}+\hat{c}_{a}^{\mu} e_{v}^{a}=0, \quad \hat{e}_{a}^{\mu} e_{v}^{a}+\hat{c}_{a}^{\mu} c_{v}^{a}=\delta_{v}^{\mu}$

one gets

$\hat{e}_{a}^{\mu}=\left(\frac{1}{1-e^{-1} c e^{-1} c} e^{-1}\right)_{a}^{\mu}$

and

$\hat{c}_{a}^{\mu}=-\left(e^{-1} c \frac{1}{1-e^{-1} c e^{-1} c} e^{-1} c e^{-1}\right)_{a}^{\mu}$.

In accord with (75) we have

$$
\begin{aligned}
e_{\mu}^{a}= & \delta_{\mu}^{a}+\frac{1}{2} h_{\mu}^{a}-\frac{1}{8}(h h+k k)_{\mu}^{a} \\
& +\frac{1}{16}\left(h^{3}+k h k+h k^{2}+k^{2} h\right)_{\mu}^{a}+\cdots, \\
\hat{e}_{a}^{\mu}= & \delta_{a}^{\mu}-\frac{1}{2} h_{a}^{\mu}+\frac{3}{8}(h h+k k)_{a}^{\mu} \\
& -\frac{5}{16}\left(h^{3}+k h k+h k^{2}+k^{2} h\right)_{a}^{\mu}+\cdots, \\
c_{\mu}^{a}= & \frac{1}{2} k_{\mu}^{a}-\frac{1}{8}(h k+k h)_{\mu}^{a} \\
& +\frac{1}{16}\left(k^{3}+h k h+h^{2} k+k h^{2}\right)_{\mu}^{a}+\cdots, \\
\hat{c}_{a}^{\mu}= & -\frac{1}{2} k_{a}^{\mu}+\frac{1}{16}(h k+k h)_{a}^{\mu} \\
& -\frac{5}{16}\left(k^{3}+h k h+h^{2} k+k h^{2}\right)_{a}^{\mu}+\cdots,
\end{aligned}
$$

or

$$
\begin{aligned}
E_{\mu}^{a}= & \delta_{\mu}^{a}+\frac{1}{2} h_{\mu}^{a}-\frac{1}{8}(h h+k k)_{\mu}^{a} \\
& +\gamma_{5}\left(\frac{1}{2} k_{\mu}^{a}-\frac{1}{8}(h k+k h)_{\mu}^{a}\right)+\cdots, \\
\hat{E}_{a}^{\mu}= & \delta_{a}^{\mu}-\frac{1}{2} h_{a}^{\mu}+\frac{3}{8}(h h+k k)_{a}^{\mu} \\
& -\gamma_{5}\left(\frac{1}{2} k_{a}^{\mu}-\frac{3}{8}(h k+k h)_{a}^{\mu}\right)+\cdots
\end{aligned}
$$

\subsection{Christoffel and Riemann}

The ordinary Christoffel symbols are

$\gamma_{\mu \nu}^{\lambda}=\frac{1}{2} g^{\lambda \rho}\left(\partial_{\mu} g_{\rho \nu}+\partial_{\nu} g_{\rho \mu}-\partial_{\rho} g_{\mu \nu}\right)$. 
The MAT Christoffel symbols are defined in a similar way

$$
\begin{aligned}
\Gamma_{\mu \nu}^{\lambda}= & \frac{1}{2} \hat{G}^{\lambda \rho}\left(\partial_{\mu} G_{\rho \nu}+\partial_{\nu} G_{\rho \mu}-\partial_{\rho} G_{\mu \nu}\right) \\
= & \frac{1}{2}\left(\hat{g}^{\lambda \rho}\left(\partial_{\mu} g_{\rho \nu}+\partial_{\nu} g_{\rho \mu}-\partial_{\rho} g_{\mu \nu}\right)\right. \\
& \left.+\hat{f}^{\lambda \rho}\left(\partial_{\mu} f_{\rho \nu}+\partial_{\nu} f_{\rho \mu}-\partial_{\rho} f_{\mu \nu}\right)\right) \\
& +\frac{1}{2} \gamma_{5}\left(\hat{g}^{\lambda \rho}\left(\partial_{\mu} f_{\rho \nu}+\partial_{\nu} f_{\rho \mu}-\partial_{\rho} f_{\mu \nu}\right)\right. \\
& \left.+\hat{f}^{\lambda \rho}\left(\partial_{\mu} g_{\rho \nu}+\partial_{\nu} g_{\rho \mu}-\partial_{\rho} g_{\mu \nu}\right)\right) \\
\equiv & \Gamma_{\mu \nu}^{(1) \lambda}+\gamma_{5} \Gamma_{\mu \nu}^{(2) \lambda} .
\end{aligned}
$$

Up to order two in $h$ and $k$ these become

$$
\begin{aligned}
\Gamma_{\mu \nu}^{(1) \lambda}= & \frac{1}{2}\left(\partial_{\mu} h_{\nu}^{\lambda}+\partial_{\nu} h_{\mu}^{\lambda}-\partial^{\lambda} h_{\mu \nu}\right. \\
& -h^{\lambda \rho}\left(\partial_{\mu} h_{\nu \rho}+\partial_{\nu} h_{\mu \rho}-\partial_{\rho} h_{\mu \nu}\right) \\
& \left.-k^{\lambda \rho}\left(\partial_{\mu} k_{\nu \rho}+\partial_{\nu} k_{\mu \rho}-\partial_{\rho} k_{\mu \nu}\right)\right)+\cdots, \\
\Gamma_{\mu \nu}^{(2) \lambda}= & \frac{1}{2}\left(\partial_{\mu} k_{\nu}^{\lambda}+\partial_{\nu} k_{\mu}^{\lambda}-\partial^{\lambda} k_{\mu \nu}\right. \\
& -h^{\lambda \rho}\left(\partial_{\mu} k_{\nu \rho}+\partial_{\nu} k_{\mu \rho}-\partial_{\rho} k_{\mu \nu}\right) \\
& \left.-k^{\lambda \rho}\left(\partial_{\mu} h_{\nu \rho}+\partial_{\nu} h_{\mu \rho}-\partial_{\rho} h_{\mu \nu}\right)\right)+\cdots .
\end{aligned}
$$

Proceeding the same way one can define the MAT Riemann tensor via $\mathcal{R}_{\mu \nu \lambda}{ }^{\rho}$ :

$$
\begin{aligned}
\mathcal{R}_{\mu \nu \lambda}{ }^{\rho}= & -\partial_{\mu} \Gamma_{\nu \lambda}^{\rho}+\partial_{\nu} \Gamma_{\mu \lambda}^{\rho}-\Gamma_{\mu \sigma}^{\rho} \Gamma_{\nu \lambda}^{\sigma}+\Gamma_{\nu \sigma}^{\rho} \Gamma_{\mu \lambda}^{\sigma} \\
= & -\partial_{\mu} \Gamma_{\nu \lambda}^{(1) \rho}+\partial_{\nu} \Gamma_{\mu \lambda}^{(1) \rho}-\Gamma_{\mu \sigma}^{(1) \rho} \Gamma_{\nu \lambda}^{(1) \sigma}+\Gamma_{\nu \sigma}^{(1) \rho} \Gamma_{\mu \lambda}^{(1) \sigma} \\
& -\Gamma_{\mu \sigma}^{(2) \rho} \Gamma_{\nu \lambda}^{(2) \sigma}+\Gamma_{\nu \sigma}^{(2) \rho} \Gamma_{\mu \lambda}^{(2) \sigma} \\
& +\gamma_{5}\left(-\partial_{\mu} \Gamma_{\nu \lambda}^{(2) \rho}+\partial_{\nu} \Gamma_{\mu \lambda}^{(2) \rho}-\Gamma_{\mu \sigma}^{(1) \rho} \Gamma_{\nu \lambda}^{(2) \sigma}\right. \\
& \left.+\Gamma_{\nu \sigma}^{(1) \rho} \Gamma_{\mu \lambda}^{(2) \sigma}-\Gamma_{\mu \sigma}^{(2) \rho} \Gamma_{\nu \lambda}^{(1) \sigma}+\Gamma_{\nu \sigma}^{(2) \rho} \Gamma_{\mu \lambda}^{(1) \sigma}\right) \\
\equiv & \mathcal{R}_{\mu \nu \lambda}^{(1) \rho}+\gamma_{5} \mathcal{R}_{\mu \nu \lambda}^{(2) \rho} .
\end{aligned}
$$

The MAT spin connection is introduced in analogy,

$$
\begin{aligned}
\Omega_{\mu}^{a b} & =E_{\nu}^{a}\left(\partial_{\mu} \hat{E}^{v b}+\hat{E}^{\sigma b} \Gamma_{\sigma \mu}^{v}\right) \\
& =\Omega_{\mu}^{(1) a b}+\gamma_{5} \Omega_{\mu}^{(2) a b}
\end{aligned}
$$

where

$$
\begin{aligned}
\Omega_{\mu}^{(1) a b}= & e_{\nu}^{a}\left(\partial_{\mu} \hat{e}^{\nu b}+\hat{e}^{\sigma b} \Gamma_{\sigma \mu}^{(1) v}+\hat{c}^{b \sigma} \Gamma_{\sigma \mu}^{(2) v}\right) \\
& +c_{\nu}^{a}\left(\partial_{\mu} \hat{c}^{v b}+\hat{e}^{\sigma b} \Gamma_{\sigma \mu}^{(2) v}+\hat{c}^{b \sigma} \Gamma_{\sigma \mu}^{(1) v}\right), \\
\Omega_{\mu}^{(2) a b}= & e_{\nu}^{a}\left(\partial_{\mu} \hat{c}^{v b}+\hat{e}^{\sigma b} \Gamma_{\sigma \mu}^{(2) v}+\hat{c}^{b \sigma} \Gamma_{\sigma \mu}^{(1) v}\right) \\
& +c_{\nu}^{a}\left(\partial_{\mu} \hat{e}^{v b}+\hat{e}^{\sigma b} \Gamma_{\sigma \mu}^{(1) v}+\hat{c}^{b \sigma} \Gamma_{\sigma \mu}^{(2) v}\right) .
\end{aligned}
$$

6.4 Transformations: diffeomorphisms

Under diffeomorphisms, $\delta x^{\mu}=\xi^{\mu}$, the Christoffel symbols transform as tensors except for one non-covariant piece,

$\delta_{\xi}^{(\text {n.c. })} \gamma_{\mu \nu}^{\lambda}=\partial_{\mu} \partial_{\nu} \xi^{\lambda}$.

The same happens for the MAT Christoffel symbols

$\delta_{\xi}^{(n . c .)} \Gamma_{\mu \nu}^{\lambda}=\partial_{\mu} \partial_{\nu} \xi^{\lambda}$.

This means in particular that $\Gamma_{\mu \nu}^{(2) \lambda}$ is a tensor.

It is more convenient to introduces also axial diffeomorphisms and use the following compact notation. The axially extended (AE) diffeomorphisms are defined by

$x^{\mu} \rightarrow x^{\mu}+\Xi^{\mu}, \quad \Xi^{\mu}=\xi^{\mu}+\gamma_{5} \zeta^{\mu}$.

Since operationally these transformations act the same way as the usual diffeomorphisms, it is easy to obtain for the noncovariant part

$\delta^{(n . c .)} \Gamma_{\mu \nu}^{\lambda}=\partial_{\mu} \partial_{\nu} \Xi^{\lambda}$.

We can also write

$\delta_{\Xi} G_{\mu \nu}=\mathcal{D}_{\mu} \Xi_{\nu}+\mathcal{D}_{\nu} \Xi_{\mu}$

where $\Xi_{\mu}=G_{\mu \nu} \Xi^{\nu}$.

In components one easily finds

$\delta_{\xi} g_{\mu \nu}=\xi^{\lambda} \partial_{\lambda} g_{\mu \nu}+\partial_{\mu} \xi^{\lambda} g_{\lambda \nu}+\partial_{\nu} \xi^{\lambda} g_{\lambda \mu}$,

$\delta_{\xi} f_{\mu \nu}=\xi^{\lambda} \partial_{\lambda} f_{\mu \nu}+\partial_{\mu} \xi^{\lambda} f_{\lambda \nu}+\partial_{\nu} \xi^{\lambda} f_{\lambda \mu}$,

$\delta_{\zeta} g_{\mu \nu}=\zeta^{\lambda} \partial_{\lambda} f_{\mu \nu}+\partial_{\mu} \zeta^{\lambda} f_{\lambda \nu}+\partial_{\nu} \zeta^{\lambda} f_{\lambda \mu}$,

$\delta_{\zeta} f_{\mu \nu}=\zeta^{\lambda} \partial_{\lambda} g_{\mu \nu}+\partial_{\mu} \zeta^{\lambda} g_{\lambda \nu}+\partial_{\nu} \zeta^{\lambda} g_{\lambda \mu}$.

Summarizing

$\delta_{\xi}^{(n . c .)} \Gamma_{\mu \nu}^{(1) \lambda}=\partial_{\mu} \partial_{\nu} \xi^{\lambda}, \quad \delta_{\xi}^{(n . c .)} \Gamma_{\mu \nu}^{(2) \lambda}=0$,

$\delta_{\zeta}^{(\text {n.c. })} \Gamma_{\mu \nu}^{(1) \lambda}=0, \quad \delta_{\zeta}^{(\text {n.c. })} \Gamma_{\mu \nu}^{(2) \lambda}=\partial_{\mu} \partial_{\nu} \zeta^{\lambda}$,

and the overall Riemann and Ricci tensors are tensor, and the Ricci scalar $\mathcal{R}$ is a scalar. But $\mathcal{R}^{(1)}$ and $\mathcal{R}^{(2)}$, separately, have the same tensorial properties.

\subsection{Transformations: Weyl transformations}

There are two types of Weyl transformations. The first is the obvious one,

$G_{\mu \nu} \longrightarrow e^{2 \omega} G_{\mu \nu}, \quad \hat{G}^{\mu \nu} \rightarrow e^{-2 \omega} \hat{G}^{\mu \nu}$,

and

$E_{\mu}^{a} \longrightarrow e^{\omega} E_{\mu}^{a}, \quad \hat{E}_{a}^{\mu} \rightarrow e^{-\omega} \hat{E}_{a}^{\mu}$.

This leads to the usual relations

$\Gamma_{\mu \nu}^{\lambda} \longrightarrow \Gamma_{\mu \nu}^{\lambda}+\partial_{\mu} \omega \delta_{\nu}^{\lambda}+\partial_{\nu} \omega \delta_{\mu}^{\lambda}-\partial_{\rho} \omega \hat{G}^{\lambda \rho} G_{\mu \nu}$ 
and

$\Omega_{\mu}^{a b} \longrightarrow \Omega_{\mu}^{a b}+\left(E_{\mu}^{a} \hat{E}^{\sigma b}-E_{\mu}^{b} \hat{E}^{\sigma a}\right) \partial_{\sigma} \omega$.

For infinitesimal $\omega$ this implies

$\delta_{\omega} g_{\mu \nu}=2 \omega g_{\mu \nu}, \quad \delta_{\omega} f_{\mu \nu}=2 \omega f_{\mu \nu}$

$\delta_{\omega}^{(0)} h_{\mu \nu}=2 \omega \eta_{\mu \nu}, \quad \delta_{\omega}^{(1)} h_{\mu \nu}=2 \omega h_{\mu \nu}, \ldots$

$\delta_{\omega}^{(0)} k_{\mu \nu}=0, \quad \delta_{\omega}^{(1)} k_{\mu \nu}=2 \omega k_{\mu \nu}, \ldots$

The second type of Weyl transformation is the axial one,

$G_{\mu \nu} \longrightarrow e^{2 \gamma_{5} \eta} G_{\mu \nu}, \quad \hat{G}^{\mu \nu} \rightarrow e^{-2 \gamma_{5} \eta} \hat{G}^{\mu \nu}$

and

$E_{\mu}^{a} \longrightarrow e^{\gamma_{5} \eta} E_{\mu}^{a}, \quad \hat{E}_{a}^{\mu} \rightarrow e^{-\gamma_{5} \eta} \hat{E}_{a}^{\mu}$.

This leads to

$\Gamma_{\mu \nu}^{\lambda} \longrightarrow \Gamma_{\mu \nu}^{\lambda}+\gamma_{5}\left(\partial_{\mu} \eta \delta_{\nu}^{\lambda}+\partial_{\nu} \eta \delta_{\mu}^{\lambda}-\partial_{\rho} \eta \hat{G}^{\lambda \rho} G_{\mu \nu}\right)$

and

$\Omega_{\mu}^{a b} \longrightarrow \Omega_{\mu}^{a b}+\gamma_{5}\left(E_{\mu}^{a} \hat{E}^{\sigma b}-E_{\mu}^{b} \hat{E}^{\sigma a}\right) \partial_{\sigma} \eta$.

Equation (109) implies

$g_{\mu \nu} \longrightarrow \cosh (2 \eta) g_{\mu \nu}+\sinh (2 \eta) f_{\mu \nu}$,

$f_{\mu \nu} \longrightarrow \cosh (2 \eta) f_{\mu \nu}+\sinh (2 \eta) g_{\mu \nu}$,

which, for infinitesimal $\eta$ becomes

$\delta_{\eta} g_{\mu \nu}=2 \eta f_{\mu \nu}, \quad \delta_{\eta}^{(0)} h_{\mu \nu}=0, \quad \delta_{\eta}^{(1)} h_{\mu \nu}=2 \eta k_{\mu \nu}, \quad \ldots$

$\delta_{\eta} f_{\mu \nu}=2 \eta g_{\mu \nu}, \quad \delta_{\eta}^{(0)} k_{\mu \nu}=2 \eta \eta_{\mu \nu}$,

$\delta_{\eta}^{(1)} k_{\mu \nu}=2 \eta h_{\mu \nu}, \quad \ldots$

6.6 Volume density

The ordinary density $\sqrt{|g|}$ is replaced by

$\sqrt{|G|}=\sqrt{\operatorname{det}(G)}=\sqrt{\operatorname{det}\left(g+\gamma_{5} f\right)}$.

The expression in the RHS has to be understood as a formal Taylor expansion in terms of the axial-complex variable $g+$ $\gamma_{5} f$. This means

$$
\begin{aligned}
\operatorname{tr} \ln \left(g+\gamma_{5} f\right)= & \operatorname{tr} \ln g+\operatorname{tr} \ln \left(1+\gamma_{5}\left(g^{-1} f\right)\right) \\
= & \operatorname{tr} \ln g+\frac{1}{2} \operatorname{tr} \ln \left(1-\left(g^{-1} f\right)^{2}\right) \\
& +\gamma_{5} \operatorname{tr} \operatorname{arcth}\left(g^{-1} f\right) \\
= & \frac{1+\gamma_{5}}{2} \operatorname{tr} \ln (g+f)+\frac{1-\gamma_{5}}{2} \operatorname{tr} \ln (g-f) .
\end{aligned}
$$

It follows that

$$
\begin{aligned}
\sqrt{|G|}= & e^{\frac{1}{2} \operatorname{tr} \ln \left(g+\gamma_{5} f\right)}=e^{\frac{1}{2}\left(\frac{1+\gamma_{5}}{2} \operatorname{tr} \ln (g+f)+\frac{1-\gamma_{5}}{2} \operatorname{tr} \ln (g-f)\right)} \\
= & \frac{1}{2}(\sqrt{\operatorname{det}(g+f)}+\sqrt{\operatorname{det}(g-f)}) \\
& +\frac{\gamma_{5}}{2}(\sqrt{\operatorname{det}(g+f)}-\sqrt{\operatorname{det}(g-f)})
\end{aligned}
$$

$\sqrt{|G|}$ has the basic property that, under diffeomorphisms,

$\delta_{\xi} \sqrt{|G|}=\xi^{\lambda} \partial_{\lambda} \sqrt{|G|}+\sqrt{|G|} \partial_{\lambda} \xi^{\lambda}$.

This is a volume density, and it has the following properties:

$\sqrt{|G|} \rightarrow e^{4 \omega} \sqrt{|G|}, \sqrt{|G|} \rightarrow e^{4 \eta \gamma_{5}} \sqrt{|G|}$,

under Weyl and axial-Weyl transformations, respectively. Moreover,

$\frac{1}{\sqrt{|G|}} \partial_{\nu} \sqrt{|G|}=\frac{1}{2} \hat{G}^{\mu \lambda} \partial_{\nu} G_{\mu \lambda}=\Gamma_{\mu \nu}^{\mu}$.

\section{Axial fermion theories}

From the above it is evident that the action for fermion a fermion field in interaction with MAT cannot be written in the classical form $\int \mathrm{d}^{4} x \sqrt{|g|} \bar{\psi} \mathcal{O} \psi$, as in the case of ordinary gravity, where $\mathcal{O}$ is the usual operatorial kinetic operator in the presence of gravity, because in the MAT case $\sqrt{\mid} G \mid$ contains the $\gamma_{5}$ matrix. Instead, $\sqrt{\mid} G \mid$ must be inserted between $\bar{\psi}$ and $\psi$. Moreover, we have to take into account that the kinetic operator contains a $\gamma$ matrix that anticommutes with $\gamma_{5}$. Thus, for instance, using $\mathcal{D}_{\lambda} G_{\mu \nu}=0$ and $\left(\mathcal{D}_{\lambda}+\frac{1}{2} \Omega_{\lambda}\right) E=0$, where $\mathcal{D}=\partial+\Gamma$, one gets

$\bar{\psi} \gamma^{a} \hat{E}_{a}^{m}\left(\partial_{m}+\frac{1}{2} \Omega_{m}\right) \psi=\bar{\psi}\left(\bar{D}_{\mu}+\frac{1}{2} \bar{\Omega}_{m}\right) \gamma^{a} \hat{E}_{a}^{m} \psi$

where a bar denotes axial-complex conjugation, i.e. a sign reversal in front of each $\gamma_{5}$ contained in the expression, for instance $\bar{\Omega}_{m}=\Omega_{m}^{(1)}-\gamma_{5} \Omega_{m}^{(2)}$. The reader should be aware that, in particular, a concise notation like $\mathcal{D}_{\mu} \gamma^{\lambda}$ is ambiguous. The MAT fermion action is now

$$
\begin{aligned}
S= & \int \mathrm{d}^{4} x i \bar{\psi} \sqrt{|\bar{G}|} \gamma^{a} \hat{E}_{a}^{m}\left(\partial_{m}+\frac{1}{2} \Omega_{m}\right) \psi \\
= & \int \mathrm{d}^{4} x i \bar{\psi} \sqrt{|\bar{G}|} \gamma^{a}\left(\hat{e}_{a}^{m}+\gamma_{5} \hat{c}_{a}^{m}\right)\left(\partial_{m}+\frac{1}{2}\left(\Omega_{m}^{(1)}+\gamma_{5} \Omega_{m}^{(2)}\right)\right) \psi \\
= & \int \mathrm{d}^{4} x \bar{\psi} \sqrt{|\bar{G}|}\left(\hat{e}_{a}^{m}-\gamma_{5} \hat{c}_{a}^{m}\right)\left[\frac{i}{2} \gamma^{a} \stackrel{\leftrightarrow}{\partial}_{m}\right. \\
& \left.+\frac{i}{4}\left(\gamma^{a} \Omega_{m}+\bar{\Omega}_{m} \gamma^{a}\right)\right] \psi \\
= & \int \mathrm{d}^{4} x \bar{\psi} \sqrt{|\bar{G}|}\left(\hat{e}_{a}^{m}-\gamma_{5} \hat{c}_{a}^{m}\right)\left[\frac{i}{2} \gamma^{a} \stackrel{\leftrightarrow}{\partial}_{m}\right.
\end{aligned}
$$




$$
\left.-\frac{1}{4} \epsilon^{a b c d}\left(\Omega_{m b c}^{(1)} \gamma_{d} \gamma_{5}+\Omega_{m b c}^{(2)} \gamma_{d}\right)\right] \psi
$$

where it is understood that $\partial_{m}$ applies only to $\psi$ or $\bar{\psi}$, as indicated, and $\bar{G}$ denotes the axial-complex conjugate. To obtain this one must use (120) and (121).

\subsection{Classical Ward identities}

Let us consider AE diffeomorphisms first, (98). It is not hard to prove that the action (122) is invariant under these transformations. Now, define the full MAT e.m. tensor by means of

$\mathbf{T}^{\mu \nu}=\frac{2}{\sqrt{|G|}} \frac{\overleftarrow{\delta} S}{\delta G_{\mu \nu}}$

This formula needs a comment, since $\sqrt{|G|}$ contains $\gamma_{5}$. To give a meaning to it we understand that the operator $\frac{2}{\sqrt{|G|}} \frac{\overleftarrow{\delta}}{\delta G_{\mu \nu}}$ in the RHS acts on the operatorial expression, say $\mathcal{O} \sqrt{|G|}$, which is inside the scalar product, i.e. $\bar{\psi} \mathcal{O} \sqrt{|G|} \psi$. Moreover, the functional derivative acts from the right of the action. Now the conservation law under diffeorphisms is

$$
\begin{aligned}
0=\delta_{\Xi} S & =\int \bar{\psi} \frac{\overleftarrow{\delta} \mathcal{O}}{\delta G_{\mu \nu}} \delta G_{\mu \nu} \psi \\
& =\int \bar{\psi} \frac{\overleftarrow{\delta} \mathcal{O}}{\delta G_{\mu \nu}}\left(\mathcal{D}_{\mu} \Xi_{\nu}+\mathcal{D}_{\nu} \Xi_{\mu}\right) \psi \\
& =-2 \int \frac{\overleftarrow{\delta} \mathcal{O}}{\delta G_{\mu \nu}} \overleftarrow{\mathcal{D}}_{\mu} \Xi_{\nu} \psi
\end{aligned}
$$

where $\mathcal{D}$ acts (from the right) on everything except the parameter $\Xi_{v}$. Differentiating with respect to the arbitrary parameters $\xi^{\mu}$ and $\zeta^{\nu}$ we obtain two conservation laws involving the two tensors

$T^{\mu \nu}=2 \bar{\psi} \frac{\overleftarrow{\delta} \mathcal{O}}{\delta G_{\mu \nu}} \psi$

$T_{5}^{\mu \nu}=2 \bar{\psi} \frac{\overleftarrow{\delta} \mathcal{O}}{\delta G_{\mu \nu}} \gamma_{5} \psi$

At the lowest order the latter are given by Eqs. (148), (149) below.

Repeating the derivation for the axial-complex Weyl transformation one can prove that, assuming for the fermion field the transformation rule

$\psi \rightarrow e^{-\frac{3}{2}\left(\omega+\gamma_{5} \eta\right)}, \psi$,

Equation (122) is invariant and we obtain the Ward identity

$0=\int \bar{\psi} \frac{\overleftarrow{\delta} \mathcal{O}}{\delta G_{\mu \nu}} G_{\mu \nu}\left(\omega+\gamma_{5} \eta\right) \psi$
We obtain in this way two WI's

$T^{\mu \nu} g_{\mu \nu}+T_{5}^{\mu \nu} f_{\mu \nu}=0$,

$T^{\mu \nu} f_{\mu \nu}+T_{5}^{\mu \nu} g_{\mu \nu}=0$,

\subsection{A simplified version}

A simplified approach to the trace anomaly calculation consists first in absorbing $\sqrt{|G|}$ in $\psi$ by setting $\Psi=|G|^{\frac{1}{4}} \psi$ and thereby assuming the transformation properties

$\delta_{\Xi} \Psi=\Xi^{\mu} \partial_{\mu} \Psi+\frac{1}{2} D_{\mu} \Xi^{\mu} \Psi$

for AE diffeomorphisms, and

$\delta_{\omega+\gamma_{5}} \Psi=e^{\frac{1}{2} \omega+\gamma_{5} \eta} \Psi$

for axial-complex Weyl transformations.

To arrive at an expanded action one uses (75) and (86), up to second order, and finds

$$
\begin{aligned}
\Omega_{\mu}^{(1) a b}= & \frac{1}{2}\left(\partial^{b} h_{\mu}^{a}-\partial^{a} h_{\mu}^{b}\right)+\frac{1}{4}\left(h^{\sigma a} \partial_{\sigma} h_{\mu}^{b}-h^{\sigma b} \partial_{\sigma} h_{\mu}^{a}\right. \\
& \left.+h^{b \sigma} \partial^{a} h_{\sigma \mu}-h^{a \sigma} \partial^{b} h_{\sigma \mu}\right) \\
& -\frac{1}{8}\left(h^{a \sigma} \partial_{\mu} h_{\sigma}^{b}-h^{b \sigma} \partial_{\mu} h_{\sigma}^{a}\right)-\frac{1}{8}\left(k^{a \sigma} \partial_{\mu} k_{\sigma}^{b}\right. \\
& \left.-k^{b \sigma} \partial_{\mu} k_{\sigma}^{a}\right)+\frac{1}{4}\left(k^{\sigma a} \partial_{\sigma} k_{\mu}^{b}-k^{\sigma b} \partial_{\sigma} k_{\mu}^{a}\right. \\
& \left.+k^{b \sigma} \partial^{a} k_{\sigma \mu}-k^{a \sigma} \partial^{b} k_{\sigma \mu}\right)+\cdots
\end{aligned}
$$

and

$$
\begin{aligned}
\Omega_{\mu}^{(2) a b}= & \frac{1}{2}\left(\partial^{b} k_{\mu}^{a}-\partial^{a} k_{\mu}^{b}\right)+\frac{1}{4}\left(h^{\sigma a} \partial_{\sigma} k_{\mu}^{b}-h^{\sigma b} \partial_{\sigma} k_{\mu}^{a}\right. \\
& \left.+h^{b \sigma} \partial^{a} k_{\sigma \mu}-h^{a \sigma} \partial^{b} k_{\sigma \mu}\right) \\
& -\frac{1}{8}\left(h^{a \sigma} \partial_{\mu} k_{\sigma}^{b}-h^{b \sigma} \partial_{\mu} k_{\sigma}^{a}\right)-\frac{1}{8}\left(k^{a \sigma} \partial_{\mu} h_{\sigma}^{b}\right. \\
& \left.-k^{b \sigma} \partial_{\mu} h_{\sigma}^{a}\right)+\frac{1}{4}\left(k^{\sigma a} \partial_{\sigma} h_{\mu}^{b}-k^{\sigma b} \partial_{\sigma} h_{\mu}^{b}\right. \\
& \left.+k^{b \sigma} \partial^{a} h_{\sigma \mu}-k^{a \sigma} \partial^{b} h_{\sigma \mu}\right)+\cdots
\end{aligned}
$$

In particular,

$\epsilon^{\mu a b c} \Omega_{\mu a b}^{(1)}=-\frac{1}{4} \epsilon^{\mu a b c}\left(h_{a}^{\sigma} \partial_{b} h_{\mu \sigma}+k_{a}^{\sigma} \partial_{b} k_{\mu \sigma}\right)+\cdots$,

$\epsilon^{\mu a b c} \Omega_{\mu a b}^{(2)}=-\frac{1}{4} \epsilon^{\mu a b c}\left(h_{a}^{\sigma} \partial_{b} k_{\mu \sigma}+k_{a}^{\sigma} \partial_{b} h_{\mu \sigma}\right)+\cdots$

Up to order two in $h$ and $k$ we have

$S=\int \mathrm{d}^{4} x \bar{\psi}|\bar{G}|^{\frac{1}{4}}\left(\hat{e}_{a}^{m}-\gamma_{5} \hat{c}_{a}^{m}\right)\left[\frac{i}{2} \gamma^{a} \stackrel{\leftrightarrow}{\partial}_{m}\right.$ 


$$
\begin{aligned}
& \left.-\frac{1}{4} \epsilon^{a b c d}\left(\Omega_{m b c}^{(1)} \gamma_{d} \gamma_{5}+\Omega_{m b c}^{(2)} \gamma_{d}\right)\right]|G|^{\frac{1}{4}} \psi \\
= & \int \mathrm{d}^{4} x\left[\frac{i}{2} \bar{\Psi} \gamma^{m} \stackrel{\leftrightarrow}{\partial}_{m} \Psi-\frac{i}{4} \bar{\Psi}\left(h_{a}^{m}-\gamma_{5} k_{a}^{m}\right) \gamma^{a} \stackrel{\leftrightarrow}{\partial}_{m} \Psi\right. \\
& +\frac{3 i}{16} \bar{\Psi}\left(\left(k^{2}\right)_{a}^{m}+\left(h^{2}\right)_{a}^{m}-\gamma_{5}(h k+k h)_{a}^{m}\right) \gamma^{a} \stackrel{\leftrightarrow}{\partial}_{m} \Psi \\
& +\frac{1}{16} \epsilon^{m a b c} \bar{\Psi}\left(\left(h_{a}^{\sigma} \partial_{b} h_{m \sigma}+k_{a}^{\sigma} \partial_{b} k_{m \sigma}\right) \gamma_{c} \gamma_{5}\right. \\
& \left.+\left(h_{a}^{\sigma} \partial_{b} k_{m \sigma}+k_{a}^{\sigma} \partial_{b} h_{m \sigma}\right) \gamma_{c}\right) \Psi+\frac{1}{8} \epsilon^{a b c d} \bar{\Psi}\left(h_{a}^{m}\right. \\
& \left.\left.-\gamma_{5} k_{a}^{m}\right)\left(\partial_{c} h_{b m} \gamma_{d} \gamma_{5}+\partial_{c} k_{b m} \gamma_{d}\right) \Psi\right]+\cdots \\
= & \int \mathrm{d}^{4} x\left[\frac{i}{2} \bar{\Psi} \gamma^{m} \stackrel{\leftrightarrow}{\partial}_{m} \Psi-\frac{i}{4} \bar{\Psi}\left(h_{a}^{m}-\gamma_{5} k_{a}^{m}\right) \gamma^{a} \stackrel{\leftrightarrow}{\partial}_{m} \Psi\right. \\
& +\frac{3 i}{16} \bar{\Psi}\left(\left(k^{2}\right)_{a}^{m}+\left(h^{2}\right)_{a}^{m}-\gamma_{5}(h k+k h)_{a}^{m}\right) \gamma^{a} \stackrel{\leftrightarrow}{\partial}_{m} \Psi \\
& -\frac{1}{16} \epsilon^{m a b c} \bar{\Psi}\left(\left(h_{a}^{\sigma} \partial_{b} h_{m \sigma}+k_{a}^{\sigma} \partial_{b} k_{m \sigma}\right) \gamma_{c} \gamma_{5}\right. \\
& \left.\left.+\left(h_{a}^{\sigma} \partial_{b} k_{m \sigma}+k_{a}^{\sigma} \partial_{b} h_{m \sigma}\right) \gamma_{c}\right) \Psi\right]+\cdots
\end{aligned}
$$

Here we do not report explicitly the terms cubic in $h$ and $k$ : they contains three powers of $h$ and/or $k$ multiplied by $\bar{\Psi} \gamma_{\mu} \Psi$ or $\bar{\Psi} \gamma_{\mu} \gamma_{5} \Psi$ and possibly by the $\epsilon$ tensor. They contain one single derivative, applied to either $h, k$ or $\Psi$. These cubic terms will not affect our results.

\subsection{Feynman rules}

For a derivation of the Feynman rules in this case see B. 3 and B.4. The fermion propagator is

$$
\frac{i}{p+i \epsilon} \text {. }
$$

The two-fermion-h-graviton vertex is $\left(V_{f f h}\right)$ :

$$
-\frac{i}{8}\left[\left(p+p^{\prime}\right)_{\mu} \gamma_{\nu}+\left(p+p^{\prime}\right)_{\nu} \gamma_{\mu}\right] \text {. }
$$

The axial two-fermion-k-graviton vertex is $\left(V_{f f k}\right)$

$$
-\frac{i}{8}\left[\left(p+p^{\prime}\right)_{\mu} \gamma_{\nu}+\left(p+p^{\prime}\right)_{\nu} \gamma_{\mu}\right] \gamma_{5}
$$

( $p$ incoming, $p^{\prime}$ outgoing). There are six two-fermion-twograviton vertices:

(1) $V_{f f h h}^{(1)}$ :

$$
\begin{aligned}
& \frac{3 i}{64}\left[\left(\left(p+p^{\prime}\right)_{\mu} \gamma_{\mu^{\prime}} \eta_{\nu v^{\prime}}+\left(p+p^{\prime}\right)_{\mu} \gamma_{\nu^{\prime}} \eta_{\nu \mu^{\prime}}+\{\mu \leftrightarrow v\}\right)\right. \\
& \left.\quad+\left(\left(p+p^{\prime}\right)_{\mu^{\prime}} \gamma_{\mu} \eta_{\nu v^{\prime}}+\left(p+p^{\prime}\right)_{\mu^{\prime}} \gamma_{\nu} \eta_{\mu v^{\prime}}+\left\{\mu^{\prime} \leftrightarrow v^{\prime}\right\}\right)\right],
\end{aligned}
$$

(2) $V_{f f k k}^{(2)}$ :

$$
\begin{aligned}
& \frac{3 i}{64}\left[\left(\left(p+p^{\prime}\right)_{\mu} \gamma_{\mu^{\prime}} \eta_{\nu v^{\prime}}+\left(p+p^{\prime}\right)_{\mu} \gamma_{v^{\prime}} \eta_{\nu \mu^{\prime}}+\{\mu \leftrightarrow \nu\}\right)\right. \\
& \left.+\left(\left(p+p^{\prime}\right)_{\mu^{\prime}} \gamma_{\mu} \eta_{\nu v^{\prime}}+\left(p+p^{\prime}\right)_{\mu^{\prime}} \gamma_{\nu} \eta_{\mu \nu^{\prime}}+\left\{\mu^{\prime} \leftrightarrow v^{\prime}\right\}\right)\right],
\end{aligned}
$$

(3) $V_{f f h k}^{(3)}$ :

$$
\begin{aligned}
& \frac{3 i}{64}\left[\left(\left(p+p^{\prime}\right)_{\mu} \gamma_{\mu^{\prime}} \eta_{v v^{\prime}}+\left(p+p^{\prime}\right)_{\mu} \gamma_{v^{\prime}} \eta_{\nu \mu^{\prime}}+\{\mu \leftrightarrow v\}\right)\right. \\
& \left.+\left(\left(p+p^{\prime}\right)_{\mu^{\prime}} \gamma_{\mu} \eta_{v v^{\prime}}+\left(p+p^{\prime}\right)_{\mu^{\prime}} \gamma_{\nu} \eta_{\mu v^{\prime}}+\left\{\mu^{\prime} \leftrightarrow v^{\prime}\right\}\right)\right] \gamma_{5},
\end{aligned}
$$

(4) $V_{f f h h}^{(1) \epsilon}$ :

$$
\frac{1}{64} t_{\mu \nu \mu^{\prime} \nu^{\prime} \kappa \lambda}\left(k-k^{\prime}\right)^{\lambda} \gamma^{\kappa} \gamma_{5}
$$

where $t$ is the tensor (34),

(5) $V_{f f k k}^{(2) \epsilon}$ :

$$
\frac{1}{64} t_{\mu \nu \mu^{\prime} v^{\prime} \kappa \lambda}\left(k-k^{\prime}\right)^{\lambda} \gamma^{\kappa} \gamma_{5},
$$

(6) $V_{f f h k}^{(3) \epsilon}$ :

$$
\frac{1}{64} t_{\mu \nu \mu^{\prime} \nu^{\prime} \kappa \lambda}\left(k-k^{\prime}\right)^{\lambda} \gamma^{\kappa} .
$$

The graviton momenta $k, k^{\prime}$ are incoming.

As anticipated above, we dispense from writing down the vertices with three $h, k$ legs. For the purposes of this paper it is possible to dispose of them with a general argument, without entering detailed calculations.

\subsection{Trace anomalies: a simplified derivation}

We will now derive the odd parity trace anomalies in the model (137), by considering only the triangle diagram contributions and disregarding tadpoles and seagull terms. We will justify later on this simplified procedure.

The overall effective action is

$$
\begin{aligned}
W[h, k]= & W[0]+\sum_{n, m=0}^{\infty} \frac{i^{m+n-1}}{2^{n+m} n ! m !} \int \prod_{i=1}^{n} \mathrm{~d} x_{i} h_{\mu_{i} v_{i}}\left(x_{i}\right) \\
& \times \prod_{j=1}^{m} \mathrm{~d} y_{j} k_{\lambda_{j} \rho_{j}}\left(y_{j}\right) \\
& \cdot\langle 0| \mathcal{T} T^{\mu_{1} v_{1}}\left(x_{1}\right) \ldots T^{\mu_{n} v_{n}}\left(x_{n}\right) \\
& \times T_{5}^{\lambda_{1} \rho_{1}}\left(y_{1}\right) \ldots T_{5}^{\lambda_{m} \rho_{m}}\left(y_{m}\right)|0\rangle
\end{aligned}
$$


where, in the simplified version of this section, the $T$ operators in the time-ordered amplitudes refer to the classical ones, i.e.

$T^{\mu v} \equiv T_{(0,0)}^{\mu \nu}=-\frac{i}{4}\left(\bar{\psi} \gamma^{\mu} \stackrel{\leftrightarrow}{\partial^{v}} \psi+\mu \leftrightarrow v\right)$

and

$$
T_{5}^{\mu \nu} \equiv T_{5(0,0)}^{\mu \nu}=\frac{i}{4}\left(\bar{\psi} \gamma_{5} \gamma^{\mu} \stackrel{\leftrightarrow}{\partial^{v}} \psi+\mu \leftrightarrow \nu\right)
$$

The quantum Ward identities for the Weyl and axial Weyl symmetry are obtained by replacing the classical e.m. tensor expressions with the one-loop one-point functions in (129) and (130)

$$
\begin{aligned}
\mathcal{T}(x) \equiv & \left\langle\left\langle T^{\mu \nu}\right\rangle\right\rangle g_{\mu \nu} \\
& +\left\langle\left\langle T_{5}^{\mu \nu}\right\rangle\right\rangle f_{\mu \nu}=0, \text { i.e. }\left\langle\left\langle T_{\mu}^{\mu}\right\rangle\right\rangle+\cdots=0
\end{aligned}
$$

and

$$
\begin{aligned}
\mathcal{T}_{5}(x) \equiv & \left\langle\left\langle T^{\mu \nu}\right\rangle\right\rangle f_{\mu \nu} \\
& +\left\langle\left\langle T_{5}^{\mu \nu}\right\rangle\right\rangle g_{\mu \nu}=0, \text { i.e. }\left\langle\left\langle T_{5 \mu}^{\mu}\right\rangle\right\rangle+\cdots=0 .
\end{aligned}
$$

In the present simplified setup the relevant one-loop onepoint functions are

$$
\begin{aligned}
\left\langle\left\langle T^{\mu \nu}(x)\right\rangle=\right. & \sum_{n, m=0}^{\infty} \frac{i^{m+n}}{2^{n+m} n ! m !} \int \prod_{i=1}^{n} \mathrm{~d} x_{i} h_{\mu_{i} \nu_{i}}\left(x_{i}\right) \prod_{j=1}^{m} \mathrm{~d} y_{j} k_{\lambda_{j} \rho_{j}}\left(y_{j}\right) \\
& \cdot\langle 0| \mathcal{T} T^{\mu \nu}(x) T^{\mu_{1} \nu_{1}}\left(x_{1}\right) \ldots T^{\mu_{n} \nu_{n}}\left(x_{n}\right) \\
& \times T_{5}^{\lambda_{1} \rho_{1}}\left(y_{1}\right) \ldots T_{5}^{\lambda_{m} \rho_{m}}\left(y_{m}\right)|0\rangle
\end{aligned}
$$

and

$$
\begin{aligned}
\left\langle\left\langle T_{5}^{\mu \nu}(x)\right\rangle=\right. & \sum_{n, m=0}^{\infty} \frac{i^{m+n}}{2^{n+m} n ! m !} \int \prod_{i=1}^{n} \mathrm{~d} x_{i} h_{\mu_{i} \nu_{i}}\left(x_{i}\right) \prod_{j=1}^{m} \mathrm{~d} y_{j} k_{\lambda_{j} \rho_{j}}\left(y_{j}\right) \\
& \cdot\langle 0| \mathcal{T} T_{5}^{\mu \nu}(x) T^{\mu_{1} \nu_{1}}\left(x_{1}\right) \ldots T^{\mu_{n} \nu_{n}}\left(x_{n}\right) \\
& \times T_{5}^{\lambda_{1} \rho_{1}}\left(y_{1}\right) \ldots T_{5}^{\lambda_{m} \rho_{m}}\left(y_{m}\right)|0\rangle .
\end{aligned}
$$

In particular for the trace anomalies, at $\mathcal{O}\left(h^{2}, h k, k^{2}\right)$ level, we have

$$
\begin{aligned}
\left\langle\left\langle T_{\mu}^{\mu}(x)\right\rangle\right\rangle^{(2)}= & -\frac{1}{8} \int \mathrm{d} x_{1} \mathrm{~d} x_{2} h_{\mu_{1} v_{1}}\left(x_{1}\right) h_{\mu_{2} v_{2}}\left(x_{2}\right) \\
& \times\left\langle 0\left|\mathcal{T} T_{\mu}^{\mu}(x) T^{\mu_{1} v_{1}}\left(x_{1}\right) T^{\mu_{2} \nu_{2}}\left(x_{2}\right)\right| 0\right\rangle \\
& -\frac{1}{4} \int \mathrm{d} x_{1} \mathrm{~d} y h_{\mu_{1} \nu_{1}}\left(x_{1}\right) k_{\lambda \rho}(y)\langle 0| \\
& \times \mathcal{T} T_{\mu}^{\mu}(x) T^{\mu_{1} \nu_{1}}\left(x_{1}\right) T_{5}^{\lambda \rho}(y)|0\rangle \\
& -\frac{1}{8} \int \mathrm{d} y_{1} \mathrm{~d} y_{2} k_{\lambda_{1} \rho_{1}}\left(y_{1}\right) k_{\lambda_{2} \rho_{2}}\left(y_{2}\right)\langle 0| \\
& \times \mathcal{T} T_{\mu}^{\mu}(x) T_{5}^{\lambda_{1} \rho_{1}}\left(y_{1}\right) T_{5}^{\lambda_{2} \rho_{2}}\left(y_{2}\right)|0\rangle
\end{aligned}
$$

and

$$
\begin{aligned}
\left.\left\langle T_{5 \mu}{ }^{\mu}(x)\right\rangle\right\rangle^{(2)}= & -\frac{1}{8} \int \mathrm{d} x_{1} \mathrm{~d} x_{2} h_{\mu_{1} v_{1}}\left(x_{1}\right) h_{\mu_{2} v_{2}}\left(x_{2}\right)\langle 0| \\
& \times \mathcal{T} T_{5 \mu}{ }^{\mu}(x) T^{\mu_{1} v_{1}}\left(x_{1}\right) T^{\mu_{2} \nu_{2}}\left(x_{2}\right)|0\rangle \\
& -\frac{1}{4} \int \mathrm{d} x \mathrm{~d} y h_{\mu_{1} v_{1}}\left(x_{1}\right) k_{\lambda \rho}(y)\langle 0| \\
& \times \mathcal{T} T_{5 \mu}{ }^{\mu}(x) T^{\mu_{1} \nu_{1}}\left(x_{1}\right) T_{5}^{\lambda \rho}(y)|0\rangle \\
& -\frac{1}{8} \int \mathrm{d} y_{1} \mathrm{~d} y_{2} k_{\lambda_{1} \rho_{1}}\left(y_{1}\right) k_{\lambda_{2} \rho_{2}}\left(y_{2}\right)\langle 0| \\
& \times \mathcal{T} T_{5 \mu}{ }^{\mu}(x) T_{5}^{\lambda_{1} \rho_{1}}\left(y_{1}\right) T_{5}^{\lambda_{2} \rho_{2}}\left(y_{2}\right)|0\rangle .
\end{aligned}
$$

It is clear that only the terms containing an odd number of $T_{5}$ will contribute to the odd parity trace anomaly.

The three-point functions (154) and (155) are given by the ordinary triangle diagrams. All such diagrams give the same contribution

$\sim\left(k_{1} \cdot k_{2} t_{\mu \nu \mu^{\prime} v^{\prime} \lambda \rho}-t_{\mu \nu \mu^{\prime} v^{\prime} \lambda \rho}^{(21)}\right) k_{1}^{\lambda} k_{2}^{\rho}$

where

$$
\begin{aligned}
t_{\mu \nu \mu^{\prime} v^{\prime} \kappa \lambda}^{(21)}= & k_{2 \mu} k_{1 \mu^{\prime}} \epsilon_{\nu v^{\prime} \kappa \lambda}+k_{2 \nu} k_{1 v^{\prime}} \epsilon_{\mu \mu^{\prime} \kappa \lambda} \\
& +k_{2 \mu} k_{1 v^{\prime}} \epsilon_{\nu \mu^{\prime} \kappa \lambda}+k_{2 v} k_{1 \mu^{\prime}} \epsilon_{\mu \nu^{\prime} \kappa \lambda} .
\end{aligned}
$$

Upon Fourier-anti-transforming and replacing in (154) we get

$$
\begin{aligned}
\left\langle\left\langle T_{\mu}^{\mu}(x)\right\rangle\right\rangle^{(2)}= & -2 N \epsilon^{\mu \nu \lambda \rho}\left(\partial_{\mu} \partial_{\sigma} h_{\nu}^{\tau} \partial_{\lambda} \partial_{\tau} k_{\rho}^{\sigma}\right. \\
& \left.-\partial_{\mu} \partial_{\sigma} h_{\nu}^{\tau} \partial_{\lambda} \partial^{\sigma} k_{\tau \rho}\right)
\end{aligned}
$$

and in (155) we get

$$
\begin{aligned}
\left\langle\left\langle T_{5 \mu}^{\mu}(x)\right\rangle\right\rangle^{(2)}= & -2 N\left[\frac { 1 } { 2 } \epsilon ^ { \mu \nu \lambda \rho } \left(\partial_{\mu} \partial_{\sigma} h_{\nu}^{\tau} \partial_{\lambda} \partial_{\tau} h_{\rho}^{\sigma}\right.\right. \\
& \left.-\partial_{\mu} \partial_{\sigma} h_{\nu}^{\tau} \partial_{\lambda} \partial^{\sigma} h_{\tau \rho}\right) \\
& \left.+\frac{1}{2} \epsilon^{\mu \nu \lambda \rho}\left(\partial_{\mu} \partial_{\sigma} k_{\nu}^{\tau} \partial_{\lambda} \partial_{\tau} k_{\rho}^{\sigma}-\partial_{\mu} \partial_{\sigma} k_{\nu}^{\tau} \partial_{\lambda} \partial^{\sigma} k_{\tau \rho}\right)\right]
\end{aligned}
$$

where $N$ is the constant that appears in front of the Pontryagin anomaly in [1], i.e. $N=\frac{i}{768 \pi^{2}}$. Covariantizing these expressions we get

$$
\begin{aligned}
\Theta_{\mu}^{\mu} \equiv \int \omega\left\langle\left\langle T_{\mu}^{\mu}(x)\right\rangle=N \int \omega \epsilon^{\mu \nu \lambda \rho} \mathcal{R}_{\mu \nu}^{(1) \sigma \tau} \mathcal{R}_{\lambda \rho \sigma \tau}^{(2)},\right. \\
\Theta_{5 \mu}{ }^{\mu} \equiv \int \eta\left\langle T_{5 \mu}{ }^{\mu}(x)\right\rangle=\frac{N}{2} \int \eta \epsilon^{\mu \nu \lambda \rho}\left(\mathcal{R}_{\mu \nu}^{(1) \sigma \tau} \mathcal{R}_{\lambda \rho \sigma \tau}^{(1)}\right. \\
\left.\quad+\mathcal{R}_{\mu \nu}^{(2) \sigma \tau} \mathcal{R}_{\lambda \rho \sigma \tau}^{(2)}\right) .
\end{aligned}
$$

The important remark is now that the odd parity trace anomaly, in an ordinary theory of Weyl fermions, can be calculated using the above theory of Dirac fermions coupled to MAT gravity and setting at the end $h_{\mu \nu} \rightarrow \frac{h_{\mu \nu}}{2}, k_{\mu \nu} \rightarrow$ $\frac{h_{\mu \nu}}{2}$ and $\omega=\eta$, for left-handed Weyl fermions, and $h_{\mu \nu} \rightarrow$ 
$\frac{h_{\mu v}}{2}, k_{\mu \nu} \rightarrow-\frac{h_{\mu v}}{2}$ for right-handed ones. We will refer to these as collapsing limits.

7.5 What happens when $h_{\mu \nu} \rightarrow \frac{h_{\mu \nu}}{2}, k_{\mu \nu} \rightarrow \frac{h_{\mu v}}{2} ?$

Let us show that in the collapsing limit $h_{\mu \nu} \rightarrow \frac{h_{\mu v}}{2}, k_{\mu \nu} \rightarrow$ $\frac{h_{\mu v}}{2}$ we have the following results:

$\Gamma_{\mu \nu}^{(1) \lambda} \rightarrow \frac{1}{2} \gamma_{\mu \nu}^{\lambda}, \quad \Gamma_{\mu \nu}^{(2) \lambda} \rightarrow \frac{1}{2} \gamma_{\mu \nu}^{\lambda}$.

This is evident in the approximate expressions (90) and (91), but it can be proved in general. To order $n$ in the expansion of $h$ and $k$ of $\Gamma_{\mu \nu}^{(1) \lambda}$ we are going to have a first term of order $n$ in $h$ alone, then $\left(\begin{array}{l}n \\ 2\end{array}\right)$ of order $n-2$ in $h$ and order 2 in $k$, then $\left(\begin{array}{l}n \\ 4\end{array}\right)$ of order $n-4$ in $h$ and order 4 in $k$, and so on, up to order $[n / 2]$ in $h$. In the collapsing limit, all these terms collapse to the first term of order $n$ in $h$ divided by $2^{n}$. In total they are

$$
\sum_{k=0}^{[n / 2]}\left(\begin{array}{c}
n \\
2 k
\end{array}\right)=2^{n-1}
$$

Therefore they give the order $n$ term in $h$ of $\gamma_{\mu \nu}^{\lambda}$ divided by 2. A similar proof holds for $\Gamma_{\mu \nu}^{(2) \lambda}$.

Looking at the definition (92) of the curvatures $\mathcal{R}_{\mu \nu \lambda}^{(1)} \rho$ and $\mathcal{R}_{\mu \nu \lambda}^{(2)} \rho$ one easily sees that in the collapsing limit

$\mathcal{R}_{\mu \nu \lambda}^{(1) \rho} \rightarrow \frac{1}{2} R_{\mu \nu \lambda}{ }^{\rho}, \quad \mathcal{R}_{\mu \nu \lambda}^{(2) \rho} \rightarrow \frac{1}{2} R_{\mu \nu \lambda}{ }^{\rho}$,

where $R_{\mu \nu \lambda}{ }^{\rho}$ is the curvature of $g_{\mu \nu}$.

In a similar way, using (133) and (134), one can show that

$\Omega_{\mu}^{(1) a b} \rightarrow \frac{1}{2} \omega_{\mu}^{a b}, \quad \Omega_{\mu}^{(2) a b} \rightarrow \frac{1}{2} \omega_{\mu}^{a b}$.

Notice also that in the collapsing limit

$g_{\mu \nu}+f_{\mu \nu}=\eta_{\mu \nu}+h_{\mu \nu}+k_{\mu \nu} \rightarrow g_{\mu \nu}$

$g_{\mu \nu}-f_{\mu \nu}=\eta_{\mu \nu}+h_{\mu \nu}-k_{\mu \nu} \rightarrow \eta_{\mu \nu}$,

so that

$\sqrt{|G|} \rightarrow \frac{1-\gamma_{5}}{2}+\frac{1+\gamma_{5}}{2} \sqrt{|g|}$

and

$E_{m}^{a} \rightarrow \delta_{m}^{a} \frac{1-\gamma_{5}}{2}+e_{m}^{a} \frac{1+\gamma_{5}}{2}$,
$\hat{E}_{a}^{m} \rightarrow \delta_{a}^{m} \frac{1-\gamma_{5}}{2}+\hat{e}_{a}^{m} \frac{1+\gamma_{5}}{2}$.
From the above follows that the action (137) tends to

$$
\begin{aligned}
S= & \int \mathrm{d}^{4} x i \bar{\Psi} \gamma^{a} \hat{E}_{a}^{m}\left(\partial_{m}+\frac{1}{2} \Omega_{m}\right) \Psi \\
& \longrightarrow \int \mathrm{d}^{4} x\left[i \bar{\Psi} \gamma^{m} \frac{1-\gamma_{5}}{2} \partial_{m} \Psi\right. \\
& \left.+i \bar{\Psi} \gamma^{a} \hat{e}_{a}^{m}\left(\partial_{m}+\frac{1}{2} \omega_{m}\right) \frac{1+\gamma_{5}}{2} \Psi\right]
\end{aligned}
$$

As for the opposite handedness one notices that, if $h_{\mu \nu} \rightarrow$ $\frac{h_{\mu v}}{2}, k_{\mu v} \rightarrow-\frac{h_{\mu v}}{2}$, we have

$\Omega_{\mu}^{(1) a b} \rightarrow \frac{1}{2} \omega_{\mu}^{a b}, \quad \Omega_{\mu}^{(2) a b} \rightarrow-\frac{1}{2} \omega_{\mu}^{a b}$,

and in (168) the sign in front of $\gamma_{5}$ is reversed. Therefore the limiting action is

$$
\begin{aligned}
S^{\prime}= & \int \mathrm{d}^{4} x\left[i \bar{\Psi} \gamma^{a} \frac{1+\gamma_{5}}{2} \partial_{a} \Psi\right. \\
& \left.+i \bar{\Psi} \gamma^{a} \hat{e}_{a}^{m}\left(\partial_{m}+\frac{1}{2} \omega_{m}\right) \frac{1-\gamma_{5}}{2} \Psi\right] .
\end{aligned}
$$

We recall that $\gamma^{a}$ is the flat (non-dynamical) gamma matrix.

Concerning the energy-momentum tensor, from the definitions (125) and (126), in the collapsing limit both $T^{\mu \nu}$ and $T_{5}^{\mu \nu}$ become

$T^{\prime \mu \nu}(x)=4 \frac{\delta S^{\prime}}{\delta h_{\mu \nu}(x)}$.

As a consequence (150) and (151) collapse to the same expression,

$\mathcal{T}(x) \rightarrow\left\langle\left\langle T^{\prime \mu \nu}\right\rangle g_{\mu \nu} \equiv \mathcal{T}^{\prime}(x)\right.$

and

$\mathcal{T}_{5}(x) \rightarrow\left\langle\left\langle T^{\prime} \mu \nu\right\rangle\right\rangle g_{\mu \nu} \equiv \mathcal{T}^{\prime}(x)$,

that is, there is only one trace Ward identity.

\subsection{The Pontryagin anomaly}

As pointed out above the odd parity trace anomaly in an ordinary theory of Weyl fermions can be calculated, to first order, using the above theory of Dirac fermions coupled to MAT gravity and calculating the collapsing limit of the Weyl anomaly for a Dirac fermion coupled to MAT gravity. The collapsing limit of the relevant action reproduces the action for Weyl fermions

$S^{\prime}=\int \mathrm{d}^{4} x \sqrt{|g|}\left[\frac{i}{2} \bar{\psi}_{L} \gamma^{m} \stackrel{\leftrightarrow}{\partial}_{m} \psi_{L}-\frac{i}{4} \omega^{\mu a b c}{\overline{\psi_{L}}}_{c} \gamma_{5} \psi_{L}\right]$

up to a right-handed kinetic term, which is, however, harmless due to the presence of the $P_{L}$ projector in the vertices. 
Inserting the replacements into either (160) or (161) we find $\mathcal{T}^{\prime}(x)=\frac{N}{4} \epsilon^{\mu \nu \lambda \rho} R_{\mu \nu}{ }^{\sigma \tau} R_{\lambda \rho \sigma \tau}$.

This is not yet the correct result for one must take into account the different combinatorics in (147) and in

$$
\begin{aligned}
W[h]= & W[0]+\sum_{n=0}^{\infty} \frac{i^{n-1}}{2^{n} n !} \int \prod_{i=1}^{n} \mathrm{~d} x_{i} h_{\mu_{i} v_{i}}\left(x_{i}\right)\langle 0| \\
& \times \mathcal{T} T^{\mu_{1} v_{1}}\left(x_{1}\right) \cdots T^{\mu_{n} v_{n}}\left(x_{n}\right)|0\rangle,
\end{aligned}
$$

which is appropriate for (175). ${ }^{9}$ This amounts to multiplying (176) by a factor of 2 . Therefore, finally the anomaly is

$\mathcal{T}(x)=\frac{N}{2} \epsilon^{\mu \nu \lambda \rho} R_{\mu \nu}{ }^{\sigma \tau} R_{\lambda \rho \sigma \tau}$,

which is the Pontrygin anomaly already found.

In the case of right-handed fermions the anomaly is the same, but with reversed sign. Thus the odd trace anomaly for Dirac fermions vanishes. This is confirmed by the following subsection.

\subsection{Odd trace anomaly in the Dirac and Majorana case}

From the results (160) and (161) we can draw other conclusions. The action (122) reduces to the usual Dirac action if we set $f_{\mu \nu}=0$, and to the Majorana action if $\psi$ satisfies the Majorana condition. From (160) we have the confirmation that the odd trace anomaly of these theories vanishes. But we also see that in both cases there is an anomaly in the axial energy-momentum tensor.

$\Theta_{5 \mu}{ }^{\mu}=\frac{N}{2} \int \eta \epsilon^{\mu \nu \lambda \rho} R_{\mu \nu}{ }^{\sigma \tau} R_{\lambda \rho \sigma \tau}$

for the Dirac case and $\frac{1}{2}$ of it in the Majorana case. This is a new result. This anomaly is the analog in the trace case of the Kimura-Delbourgo-Salam anomaly for the axial current.

\section{Odd trace anomalies (the complete calculation)}

Now we would like to justify the assumption made above, according to which only triangle diagrams provide a nonvanishing contribution to the odd trace anomaly. The complete calculation requires taking into account all the tadpoles and seagull terms that arise from the action (122). We start with the quantum Ward identity (150) and (151).

\footnotetext{
9 The factor $\frac{1}{2^{n}}$ in the RHS must be properly interpreted. When inserting the results for the $n$-point functions in (177), one should recall that the vertex (139) contains already a $\frac{1}{2}$ factor in it with respect to the e.m. tensor: symbolically we could write $V_{f f h}=\frac{1}{2} \tilde{T}$, where $\tilde{T}$ is the Fourier transform of the e.m. tensor with fields replaced by corresponding plane waves. A simple practical recipe is to just forget factor $\frac{1}{2^{n}}$ in (177), as was done, somewhat sloppily, in [1]. The same holds for Eq. (147).
}

\subsection{Trace Ward indentity}

We need to expand this Ward identity in series of $h$ and $k$. The expanded versions is written down in Appendix B.5. Since eventually we are interested only in the odd terms we will drop all the terms that we already know are even or vanish (the vev of $T_{(0,0)}^{\mu \nu}(x)$ and $T_{5(0,0)}^{\mu \nu}(x)$, the two-point functions of the em and axial em tensor, as well as the vev of the second and third derivatives of $S$ ). In this way the WI's get simplified as follows:

$\begin{aligned} \mathcal{T}_{(1,1)}\left(x, x_{1}, y_{1}\right) \equiv & \mathcal{T}_{(1,1)} \underset{\mu}{\mu \mu_{1} v_{1} \lambda_{1} \rho_{1}}\left(x, x_{1}, y_{1}\right)=0, \\ \mathcal{T}_{(2,0)}\left(x, x_{1}, x_{2}\right) \equiv & \mathcal{T}_{(2,0)}^{\mu \mu_{1} v_{1} \mu_{2} v_{2}}\left(x, x_{1}, x_{2}\right)=0, \\ \mathcal{T}_{(0,2)}\left(x, y_{1}, y_{2}\right) \equiv & \mathcal{T}_{(0,2) \mu}^{\mu \lambda_{1} \rho_{1} \lambda_{2} \rho_{2}}\left(x, y_{1}, y_{2}\right)=0 \\ & \cdots,\end{aligned}$

and

$$
\begin{aligned}
& \mathcal{T}_{5(1,1)}\left(x, x_{1}, y_{1}\right) \equiv \mathcal{T}_{5(1,1)} \mu_{\mu}^{\mu \mu_{1} v_{1} \lambda_{1} \rho_{1}}\left(x, x_{1}, y_{1}\right)=0, \\
& \mathcal{T}_{5(2,0)}\left(x, x_{1}, x_{2}\right) \equiv \mathcal{T}_{5(2,0)} \mu_{\mu} \mu_{1} v_{1} \mu_{2} v_{2}\left(x, x_{1}, x_{2}\right)=0, \\
& \mathcal{T}_{5(0,2)}\left(x, y_{1}, y_{2}\right) \equiv \mathcal{T}_{5(0,2) \mu}{ }^{\mu \lambda_{1} \rho_{1} \lambda_{2} \rho_{2}}\left(x, y_{1}, y_{2}\right)=0
\end{aligned}
$$

These are the Ward identities in the absence of anomalies, but we expect the RHSs of all these identities to be in fact different from zero at one-loop. The odd parity anomaly can be present only in the RHSs of (180), (184) and (185): the remaining two cannot contain the $\epsilon$ tensor linearly. After such a repeated trimming, the relevant WI for our purposes are $(180,184)$ and $(185)$, and the terms that need to be closely scrutinized are

$$
\begin{aligned}
\mathcal{T}_{(1,1)}^{\mu \nu \mu_{1} \nu_{1} \lambda_{1} \rho_{1}}\left(x, x_{1}, y_{1}\right) & \\
= & -\left\langle 0\left|\mathcal{T} T_{(0,0)}^{\mu \nu}(x) T_{(0,0)}^{\mu_{1} \nu_{1}}\left(x_{1}\right) T_{5(0,0)}^{\lambda_{1} \rho_{1}}\left(y_{1}\right)\right| 0\right\rangle \\
& +4 i\left\langle 0\left|\mathcal{T} T_{5(0,0)}^{\lambda \rho_{1}}\left(y_{1}\right) \frac{\delta^{2} S}{\delta h_{\mu \nu}(x) \delta h_{\mu_{1} \nu_{1}}\left(x_{1}\right)}\right| 0\right\rangle \\
& +4 i\left\langle 0\left|\mathcal{T} T_{(0,0)}^{\mu_{1} \nu_{1}}\left(x_{1}\right) \frac{\delta^{2} S}{\delta k_{\lambda_{1} \rho_{1}}\left(y_{1}\right) \delta h_{\mu \nu}(x)}\right| 0\right\rangle \\
& +4 i\left\langle 0\left|\mathcal{T} T_{(0,0)}^{\mu \nu}(x) \frac{\delta^{2} S}{\delta k_{\lambda_{1} \rho_{1}}\left(y_{1}\right) \delta k_{\mu_{1} \nu_{1}}\left(x_{1}\right)}\right| 0\right\rangle,
\end{aligned}
$$

together with

$$
\begin{aligned}
& \mathcal{T}_{5(2,0)}^{\lambda \rho \mu_{1} \nu_{1} \mu_{2} v_{2}}\left(x, x_{1}, x_{2}\right) \\
& =-\left\langle 0\left|\mathcal{T} T_{5(0,0)}^{\lambda \rho}(x) T_{(0,0)}^{\mu_{1} \nu_{1}}\left(x_{1}\right) T_{(0,0)}^{\mu_{2} \nu_{2}}\left(x_{2}\right)\right| 0\right\rangle \\
& \quad+4 i\left\langle 0\left|\mathcal{T} T_{(0,0)}^{\mu_{1} v_{1}}\left(x_{1}\right) \frac{\delta^{2} S}{\delta k_{\lambda \rho}(x) \delta h_{\mu_{2} \nu_{2}}\left(x_{2}\right)}\right| 0\right\rangle \\
& \quad+4 i\left\langle 0\left|\mathcal{T} T_{(0,0)}^{\mu_{2} \nu_{2}}\left(x_{2}\right) \frac{\delta^{2} S}{\delta h_{\mu_{1} v_{1}}\left(x_{1}\right) \delta k_{\lambda \rho}(x)}\right| 0\right\rangle \\
& \quad+4 i\left\langle 0\left|\mathcal{T} T_{5(0,0)}^{\lambda \rho}(x) \frac{\delta^{2} S}{\delta h_{\mu_{1} v_{1}}\left(x_{1}\right) \delta h_{\mu_{2} v_{2}}\left(x_{2}\right)}\right| 0\right\rangle
\end{aligned}
$$


and

$$
\begin{aligned}
\mathcal{T}_{5(0,2)}^{\lambda \rho \lambda_{1} \rho_{1} \lambda_{2} \rho_{2}}\left(x, y_{1}, y_{2}\right) & \\
= & -\left\langle 0\left|\mathcal{T} T_{5(0,0)}^{\lambda \rho}(x) T_{5(0,0)}^{\lambda_{1} \rho_{1}}\left(y_{1}\right) T_{5(0,0)}^{\lambda_{2} \rho_{2}}\left(y_{2}\right)\right| 0\right\rangle \\
& +4 i\left\langle 0\left|\mathcal{T} T_{5(0,0)}^{\lambda_{1} \rho_{1}}\left(y_{1}\right) \frac{\delta^{2} S}{\delta k_{\lambda \rho}(x) \delta k_{\lambda_{2} \rho_{2}}\left(y_{2}\right)}\right| 0\right\rangle \\
& +4 i\left\langle 0\left|\mathcal{T} T_{5(0,0)}^{\lambda_{2} \rho_{2}}\left(y_{2}\right) \frac{\delta^{2} S}{\delta k_{\lambda_{1} \rho_{1}}\left(y_{1}\right) \delta k_{\lambda \rho}(x)}\right| 0\right\rangle \\
& +4 i\left\langle 0\left|\mathcal{T} T_{5(0,0)}^{\lambda \rho}(x) \frac{\delta^{2} S}{\delta k_{\lambda_{1} \rho_{1}}\left(y_{1}\right) \delta k_{\lambda_{2} \rho_{2}}\left(y_{2}\right)}\right| 0\right\rangle .
\end{aligned}
$$

The terms above that contain the second derivative of $S$ are bubble diagrams where one vertex has two external $h$ and/or $k$ graviton lines. These diagrams are similar to those already met above and in [1], and can be shown to similarly vanish; see Appendices C.1.1 and C.1.2. Therefore we are left with

$\mathcal{T}_{(1,1)}\left(x, x_{1}, y_{1}\right)=-\left\langle 0\left|\mathcal{T} T_{(0,0) \mu}^{\mu}(x) T_{(0,0)}^{\mu_{1} \nu_{1}}\left(x_{1}\right) T_{5(0,0)}^{\lambda_{1} \rho_{1}}\left(y_{1}\right)\right| 0\right\rangle$,

$\mathcal{T}_{5(2,0)}\left(x, x_{1}, x_{2}\right)=-\left\langle 0\left|\mathcal{T} T_{5(0,0)}^{\lambda}(x) T_{(0,0)}^{\mu_{1} \nu_{1}}\left(x_{1}\right) T_{(0,0)}^{\mu_{2} \nu_{2}}\left(x_{2}\right)\right| 0\right\rangle$,

$\mathcal{T}_{5(0,2)}\left(x, y_{1}, y_{2}\right)=-\left\langle 0\left|\mathcal{T} T_{5(0,0) \lambda}^{\lambda}(x) T_{5(0,0)}^{\lambda_{1} \rho_{1}}\left(y_{1}\right) T_{5(0,0)}^{\lambda_{2} \rho_{2}}\left(y_{2}\right)\right| 0\right\rangle$,

which are the intermediate results already obtained above. From this point on the calculation proceeds as in Sect. 7.4.

\section{Conclusion}

In this paper we have dealt with two subjects: the odd parity trace anomaly in chiral fermion theories in a $4 \mathrm{~d}$ curved background and the introduction of an axial 'metric' beside the familiar gravity metric. We have recalculated the first with the Feynman diagram method in a more complete way, by including in the computation also tadpole and seagull terms. We have verified that the latter do not modify the result of [1]. To do so we have also recalculated the Ward identity for diffeomorphims. In this paper we have constantly been using DR, leaving to a future investigation the discussion of other regularizations. The other important topic of this paper is the introduction of MAT (metric-axial-metric) gravity and the relevant formalism. MAT gravity may have of course an autonomous development and could be studied as a new bimetric model, with the new characteristics that it interacts also axially with fermions. We postpone this analysis to a future work. In this paper we have utilized MAT gravity in order to disentangle the thorny issue of the path integral measure in a theory of chiral fermions. In fact MAT gravity interact naturally with Dirac fermions. We have shown that one can compute the trace anomalies of a theory of Dirac fermions coupled to a background MAT gravity, and then recover the results for a chiral fermion theory coupled to ordinary gravity by simply taking a (smooth) limit. We have shown that in this way one obtains the same results as in [1].

Finally, let us remark that in this paper we did not verify the Ward identity for two types of diffeomorphisms in MAT background, much as was done in Sect. 4. From consolidated experience we believe that this will not modify the trace anomalies of the model, but the problem is interesting in itself. Can there be anomalies of the Einstein-Lorentz type in one of the Ward identities? This is an intriguing problem we leave for the future.

We are aware that the result of the present and previous papers contradicts a consolidated wisdom in the literature. Therefore we do not believe our result is completely settled. As we have already pointed out, further investigations are necessary in order to confirm or disprove it.

Acknowledgements LB would like to thank the Yukawa Institute for Theoretical Physics, Kyoto and the KEK Theory Center, KEK, Tsukuba, where he carried out most of this research, for their kind hospitality and support. We would like to thank Fiorenzo Bastianelli for a useful exchange of messages. This research has been supported by the Croatian Science Foundation under the Project No. 8946 and by the University of Rijeka under the research support No. 13.12.1.4.05. Finally, ADP is grateful to CAPES and CNPq for support.

Open Access This article is distributed under the terms of the Creative Commons Attribution 4.0 International License (http://creativecomm ons.org/licenses/by/4.0/), which permits unrestricted use, distribution, and reproduction in any medium, provided you give appropriate credit to the original author(s) and the source, provide a link to the Creative Commons license, and indicate if changes were made. Funded by $\mathrm{SCOAP}^{3}$.

\section{Appendices}

\section{A The triangle diagram}

In this appendix we derive in more detail the result of [1]. Employing the Feynman rules of the free chiral fermion coupled to an external gravitational field, the contribution from the triangle diagram is expressed as

$$
\begin{aligned}
T_{\mu \nu \mu^{\prime} \nu^{\prime}}\left(k_{1}, k_{2}\right)= & \int \frac{\mathrm{d}^{4} p}{(2 \pi)^{4}} \operatorname{Tr}\left\{\frac { i } { 8 } \left[\left(2 p-k_{1}\right)_{\mu} \gamma_{\nu}\right.\right. \\
& +(\mu \leftrightarrow v)]\left(\frac{1+\gamma_{5}}{2}\right) \frac{i}{\left(\not p-\not k_{1}\right)+i \epsilon} \\
& \times \frac{i}{8}\left[\left(2 p-2 k_{1}-k_{2}\right)_{\mu^{\prime}} \gamma_{v^{\prime}}\right. \\
& \left.+\left(\mu^{\prime} \leftrightarrow v^{\prime}\right)\right]\left(\frac{1+\gamma_{5}}{2}\right) \frac{i}{\left(\not p-\not k_{1}-\not k_{2}\right)+i \epsilon} \\
& \left.\times \frac{i}{4}\left(2 \not p-\not k_{1}-\not k_{2}\right)\left(\frac{1+\gamma_{5}}{2}\right) \frac{i}{\not p+i \epsilon}\right\} .
\end{aligned}
$$


Using the properties of the gamma matrices, one obtains ${ }^{10}$

$$
\begin{aligned}
T_{\mu \nu \mu^{\prime} \nu^{\prime}}\left(k_{1}, k_{2}\right)= & -\frac{1}{256} \int \frac{\mathrm{d}^{4} p}{(2 \pi)^{4}} \operatorname{Tr} \\
& \times\left\{\left[\frac{\not p}{p^{2}}\left(2 p-k_{1}\right)_{\mu} \gamma_{\nu}+(\mu \leftrightarrow v)\right]\right. \\
& \times \frac{\left(\not p-\not k_{1}\right)}{\left(p-k_{1}\right)^{2}}\left[\left(2 p-2 k_{1}-k_{2}\right)_{\mu^{\prime}} \gamma_{v^{\prime}}\right. \\
& \left.+\left(\mu^{\prime} \leftrightarrow v^{\prime}\right)\right] \frac{\left(\not p-\not k_{1}-\not k_{2}\right)}{\left(p-k_{1}-k_{2}\right)^{2}} \\
& \left.\times\left(2 \not p-\not k_{1}-k_{2}\right)\left(\frac{1+\gamma_{5}}{2}\right)\right\} .
\end{aligned}
$$

Clearly, such an integral is ultraviolet divergent. In order to proceed with the computation, we employ dimensional regularization, where additional components are added to the momentum, namely, $p \rightarrow p+\ell$, where $\ell=\left(\ell_{4}, \ldots, \ell_{n-4}\right)$. This implies, in particular,

$\gamma^{\mu} p_{\mu} \longrightarrow \gamma^{\mu} p_{\mu}+\gamma^{\bar{\mu}} \ell_{\bar{\mu}}$

with $\bar{\mu} \in\{4, \ldots, n-4\}$. Hence, Eq. (193) is replaced by

$$
\begin{aligned}
& T_{\mu \nu \mu^{\prime} \nu^{\prime}}\left(k_{1}, k_{2}\right) \\
&=-\frac{1}{256} \int \frac{\mathrm{d}^{4} p}{(2 \pi)^{4}} \int \frac{d^{n-4} \ell}{(2 \pi)^{n-4}} \operatorname{Tr} \\
& \times\left\{\left[\frac{\not p+\ell}{p^{2}-\ell^{2}}\left(2 p-k_{1}\right)_{\mu} \gamma_{\nu}+(\mu \leftrightarrow v)\right] \frac{\left(\not p+\ell-k_{1}\right)}{\left(p-k_{1}\right)^{2}-\ell^{2}}\right. \\
& \times\left[\left(2 p-2 k_{1}-k_{2}\right)_{\mu^{\prime}} \gamma_{\nu^{\prime}}+\left(\mu^{\prime} \leftrightarrow \nu^{\prime}\right)\right] \\
& \times \underbrace{\frac{(\not p+\ell-\not k 1}{\left(p-k_{1}-k_{2}\right)^{2}-\ell^{2}}\left(2 \not p+2 \ell-\not k_{1}-\not k_{2}\right)}_{(*)} \\
&\left.\times\left(\frac{1+\gamma_{5}}{2}\right)\right\} .
\end{aligned}
$$

Equation (195) is now regularized and we can continue with the computation of the diagram. In order to simplify our analysis a bit, we ignore the identity in the projector $\left(1+\gamma_{5}\right) / 2$ since we are concerned with the parity odd part contribution of the diagram, which is encoded in the $\gamma_{5}$ sector. Also, we omit the symmetrizations in $(\mu \leftrightarrow v)$ and in $\left(\mu^{\prime} \leftrightarrow v^{\prime}\right)$ for the time being and reintroduce them later on.

Let us take the term (*) and define $q=k_{1}+k_{2}$. It is simple to check that

$$
\begin{aligned}
(*) & =\frac{(\not p+\ell-q)}{(p-q)^{2}-\ell^{2}}(2 \not p+2 \ell-q) \\
& =1+\frac{\not p-\ell}{\not p+\ell-q}+\frac{2 \ell}{\not p+\ell-q},
\end{aligned}
$$

\footnotetext{
${ }^{10}$ We have dropped the $i \epsilon$ factor in the denominators, for convenience.
}

and plugging it into Eq. (195), one ends up with

$$
\begin{aligned}
T_{\mu \nu \mu^{\prime} \nu^{\prime}}\left(k_{1}, k_{2}\right)= & T_{\mu \nu \mu^{\prime} v^{\prime}}^{(1)}\left(k_{1}, k_{2}\right)+T_{\mu \nu \mu^{\prime} v^{\prime}}^{(2)}\left(k_{1}, k_{2}\right) \\
& +\tilde{T}_{\mu \nu \mu^{\prime} \nu^{\prime}}\left(k_{1}, k_{2}\right),
\end{aligned}
$$

with

$$
\begin{aligned}
& T_{\mu \nu \mu^{\prime} \nu^{\prime}}^{(1)}\left(k_{1}, k_{2}\right)=-\frac{1}{256} \int \frac{\mathrm{d}^{4} p}{(2 \pi)^{4}} \int \frac{d^{n-4} \ell}{(2 \pi)^{n-4}} \\
& \times \operatorname{Tr}\left[\frac{\not p+\ell}{p^{2}-\ell^{2}}\left(2 p-k_{1}\right)_{\mu} \gamma_{\nu} \frac{\left(\not p+\ell-\not k_{1}\right)}{\left(p-k_{1}\right)^{2}-\ell^{2}}\right. \\
& \left.\times\left(2 p-2 k_{1}-k_{2}\right)_{\mu^{\prime}} \gamma_{\nu^{\prime}} \frac{\gamma_{5}}{2}\right], \\
& T_{\mu \nu \mu^{\prime} v^{\prime}}^{(2)}\left(k_{1}, k_{2}\right)=-\frac{1}{256} \int \frac{\mathrm{d}^{4} p}{(2 \pi)^{4}} \int \frac{d^{n-4} \ell}{(2 \pi)^{n-4}}
\end{aligned}
$$

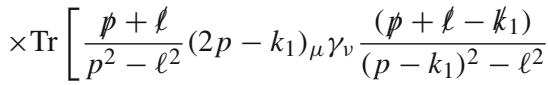

$$
\begin{aligned}
& \left.\times\left(2 p-2 k_{1}-k_{2}\right)_{\mu^{\prime}} \gamma_{v^{\prime}} \frac{(\not p-\ell)}{\not p+\ell-q} \frac{\gamma_{5}}{2}\right] \text {, } \\
& \tilde{T}_{\mu \nu \mu^{\prime} \nu^{\prime}}\left(k_{1}, k_{2}\right)=-\frac{1}{256} \int \frac{\mathrm{d}^{4} p}{(2 \pi)^{4}} \int \frac{d^{n-4} \ell}{(2 \pi)^{n-4}} \\
& \times \operatorname{Tr}\left[\frac{\not p+\ell}{p^{2}-\ell^{2}}\left(2 p-k_{1}\right)_{\mu} \gamma_{v} \frac{(\not p+\ell-\not k 1)}{\left(p-k_{1}\right)^{2}-\ell^{2}}\right. \\
& \left.\times\left(2 p-2 k_{1}-k_{2}\right)_{\mu^{\prime}} \gamma_{\nu^{\prime}} \frac{\ell}{\not p+\ell-q} \gamma_{5}\right] \text {. }
\end{aligned}
$$

We detail the computation of each contribution $T^{(1)}, T^{(2)}$ and $\tilde{T}$ in the following lines.

A.1 $T_{\mu \nu \mu^{\prime} v^{\prime}}^{(1)}\left(k_{1}, k_{2}\right)$

The contribution $T^{(1)}$ can be expressed as

$$
\begin{aligned}
T_{\mu \nu \mu^{\prime} \nu^{\prime}}^{(1)}\left(k_{1}, k_{2}\right)= & -\frac{1}{256} \int \frac{\mathrm{d}^{4} p}{(2 \pi)^{4}} \\
& \times \int \frac{d^{n-4} \ell}{(2 \pi)^{n-4}} \frac{\left(2 p-k_{1}\right)_{\mu}\left(2 p-2 k_{1}-k_{2}\right)_{\mu^{\prime}}}{2\left(p^{2}-\ell^{2}\right)\left[\left(p-k_{1}\right)^{2}-\ell^{2}\right]} \\
& \times \underbrace{\operatorname{Tr}\left[(\not p+\ell) \gamma_{\nu}\left(\not p+\ell-\not k \gamma_{1}\right) \gamma_{\nu^{\prime}} \gamma_{5}\right]}_{4 i p^{\alpha} k_{1}^{\beta} \epsilon_{\alpha \nu \beta \nu^{\prime}}} .
\end{aligned}
$$

Employing the Feynman parametrization, Eq. (199) is written as

$$
\begin{aligned}
& T_{\mu \nu \mu^{\prime} \nu^{\prime}}^{(1)}\left(k_{1}, k_{2}\right)=-\frac{i}{128} \int \frac{\mathrm{d}^{4} p}{(2 \pi)^{4}} \int \frac{d^{n-4} \ell}{(2 \pi)^{n-4}} \\
& \quad \times \int_{0}^{1} \mathrm{~d} x \frac{\left(2 p-k_{1}\right)_{\mu}\left(2 p-2 k_{1}-k_{2}\right)_{\mu^{\prime}}}{\left\{\left[\left(p-k_{1}\right)^{2}-\ell^{2}\right] x+(1-x)\left(p^{2}-\ell^{2}\right)\right\}^{2}} p^{\alpha} k_{1}^{\beta} \epsilon_{\alpha \nu \beta \nu^{\prime}} .
\end{aligned}
$$

Performing the shift $p \rightarrow p+x k_{1}$ and taking into account that just even powers of $p$ in the numerator will result on non-vanishing contributions to $T^{(1)}$, one obtains 


$$
\begin{aligned}
& T_{\mu \nu \mu^{\prime} \nu^{\prime}}^{(1)}\left(k_{1}, k_{2}\right) \\
& =\frac{i}{128} \int_{0}^{1} \mathrm{~d} x \int \frac{d^{n-4} \ell}{(2 \pi)^{n-4}} \\
& \quad \times \int \frac{\mathrm{d}^{4} p}{(2 \pi)^{4}} \frac{2 p_{\mu^{\prime}}(1-2 x) k_{1 \mu}+2 p_{\mu}\left[2(1-x) k_{1}+k_{2}\right]_{\mu}}{\left[p^{2}+x(1-x) k_{1}^{2}-\ell^{2}\right]^{2}} p^{\alpha} k_{1}^{\beta} \epsilon_{\alpha \nu \beta \nu^{\prime}} .
\end{aligned}
$$

Making use of Lorentz symmetry, one can make the following replacement:

$p^{\mu} p^{\nu} \longrightarrow \frac{1}{4} \eta^{\mu \nu} p^{2}$,

which gives rise to

$$
\begin{aligned}
& T_{\mu \nu \mu^{\prime} \nu^{\prime}}^{(1)}\left(k_{1}, k_{2}\right) \\
& =\frac{i}{256} \int_{0}^{1} \mathrm{~d} x \int \frac{d^{n-4} \ell}{(2 \pi)^{n-4}} \\
& \quad \times \int \frac{\mathrm{d}^{4} p}{(2 \pi)^{4}} \frac{\delta_{\mu^{\prime}}^{\alpha}(1-2 x) k_{1 \mu}+\delta_{\mu}^{\alpha}\left[2(1-x) k_{1}+k_{2}\right]_{\mu^{\prime}}}{\left[p^{2}+x(1-x) k_{1}^{2}-\ell^{2}\right]^{2}} p^{2} k_{1}^{\beta} \epsilon_{\alpha \nu \beta \nu^{\prime}} .
\end{aligned}
$$

After taking into account the contraction of the Kronecker deltas with the $\epsilon$-tensor and imposing the symmetrization of $(\mu \leftrightarrow v)$ and $\left(\mu^{\prime} \leftrightarrow v^{\prime}\right)$ one immediately sees that the contribution from $T^{(1)}$ vanishes.

$$
\begin{aligned}
& \text { A.2 } T_{\mu \nu \mu^{\prime} v^{\prime}}^{(2)}\left(k_{1}, k_{2}\right) \\
& T_{\mu \nu \mu^{\prime} v^{\prime}}^{(2)}\left(k_{1}, k_{2}\right) \\
& =\frac{1}{256} \int \frac{\mathrm{d}^{4} p}{(2 \pi)^{4}} \int \frac{d^{n-4} \ell}{(2 \pi)^{n-4}} \frac{\left(2 p+k_{1}\right)_{\mu}\left(2 p-k_{2}\right)_{\mu^{\prime}}}{2\left(p^{2}-\ell^{2}\right)\left[\left(p-k_{2}\right)^{2}-\ell^{2}\right]} \\
& \quad \times \underbrace{\operatorname{Tr}\left[\gamma_{v}(\not p+\ell) \gamma_{v^{\prime}}\left(\not p+\ell-\not k_{2}\right) \gamma_{5}\right]}_{4 i p^{\alpha} k_{2}^{\beta} \epsilon_{v \alpha \nu^{\prime} \beta}} .
\end{aligned}
$$

As before, one employs the Feynman parametrization and in very strict analogy, perform the shift $p \rightarrow p+x k_{2}$. This renders

$$
\begin{aligned}
& T_{\mu \nu \mu^{\prime} \nu^{\prime}}^{(2)}\left(k_{1}, k_{2}\right)=\frac{i}{128} \int_{0}^{1} \mathrm{~d} x \int \frac{d^{n-4} \ell}{(2 \pi)^{n-4}} \\
& \quad \times \int \frac{\mathrm{d}^{4} p}{(2 \pi)^{4}} \frac{\left(2 p+k_{1}+2 x k_{2}\right)_{\mu}\left(2 p-(1-x) k_{2}\right)_{\mu^{\prime}}}{\left[p^{2}-\ell^{2}-x(x-1) k_{2}^{2}\right]^{2}} \\
& \quad \times p^{\alpha} k_{2}^{\beta} \epsilon_{\nu \alpha \nu^{\prime} \beta} .
\end{aligned}
$$

Collecting just the even powers of $p$ in the numerator of (205) and applying Eq. (202), one immediately obtains

$$
\begin{aligned}
& T_{\mu \nu \mu^{\prime} \nu^{\prime}}^{(2)}\left(k_{1}, k_{2}\right)=\frac{i}{256} \int_{0}^{1} \mathrm{~d} x \int \frac{d^{n-4} \ell}{(2 \pi)^{n-4}} \\
& \quad \times \int \frac{\mathrm{d}^{4} p}{(2 \pi)^{4}} \frac{\delta_{\mu}^{\alpha}(x-1) k_{2 \mu^{\prime}}+\delta_{\mu^{\prime}}^{\alpha}\left(k_{1}+2 x k_{2}\right)_{\mu}}{\left[p^{2}-\ell^{2}-x(x-1) k_{2}^{2}\right]^{2}} k_{2}^{\beta} \epsilon_{\nu \alpha \nu^{\prime} \beta} .
\end{aligned}
$$

For the same reasons as described in the previous subsection, after symmetrizations, the contribution from $T^{(2)}$ vanishes.

\section{A.3 $\tilde{T}_{\mu v \mu^{\prime} v^{\prime}}\left(k_{1}, k_{2}\right)$}

$$
\begin{aligned}
& \tilde{T}_{\mu \nu \mu^{\prime} \nu^{\prime}}\left(k_{1}, k_{2}\right)=-\frac{1}{256} \int \frac{\mathrm{d}^{4} p}{(2 \pi)^{4}} \int \frac{d^{n-4} \ell}{(2 \pi)^{n-4}} \\
& \times \frac{\left(2 p-k_{1}\right)_{\mu}\left(2 p-2 k_{1}-k_{2}\right)_{\mu^{\prime}}}{\left(p^{2}-\ell^{2}\right)\left[\left(p-k_{1}\right)^{2}-\ell^{2}\right]\left[(p-q)^{2}-\ell^{2}\right]}
\end{aligned}
$$

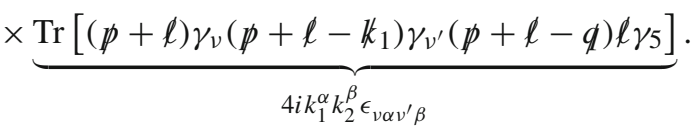

The Feynman parametrization leads to

$$
\begin{aligned}
& \tilde{T}_{\mu \nu \mu^{\prime} \nu^{\prime}}\left(k_{1}, k_{2}\right)=-\frac{i}{32} k_{1}^{\alpha} k_{2}^{\beta} \epsilon_{\nu \alpha \nu^{\prime} \beta} \int \frac{\mathrm{d}^{4} p}{(2 \pi)^{4}} \int \frac{d^{n-4} \ell}{(2 \pi)^{n-4}} \int_{0}^{1} \mathrm{~d} x \\
& \quad \times \int_{0}^{1-x} \mathrm{~d} y \frac{\left(2 p-k_{1}\right)_{\mu}\left(2 p-2 k_{1}-k_{2}\right)_{\mu^{\prime}}}{\left\{\left[\left(p-k_{1}\right)^{2}-\ell^{2}\right] x+\left[(p-q)^{2}-\ell^{2}\right] y+\left(p^{2}-\ell^{2}\right)(1-x-y)\right\}^{3}} \ell^{2} .
\end{aligned}
$$

Making the shift $p \rightarrow p+x k_{1}+y q$ and a few algebraic manipulations, Eq. (208) becomes

$$
\begin{aligned}
& \tilde{T}_{\mu \nu \mu^{\prime} \nu^{\prime}}\left(k_{1}, k_{2}\right)=-\frac{i}{32} k_{1}^{\alpha} k_{2}^{\beta} \epsilon_{\nu \alpha \nu^{\prime} \beta} \int_{0}^{1} \mathrm{~d} x \int_{0}^{1-x} \mathrm{~d} y \int \frac{\mathrm{d}^{4} p}{(2 \pi)^{4}} \\
& \times \int \frac{d^{n-4} \ell}{(2 \pi)^{n-4}} \frac{\left(2 p+2 x k_{1}+2 y q-k_{1}\right)_{\mu}\left(2 p+2 x k_{1}+2 y q-2 k_{1}-k_{2}\right)_{\mu^{\prime}}}{\left[p^{2}-\ell^{2}+2 k_{1} \cdot k_{2} y(1-y-x)\right]^{3}} .
\end{aligned}
$$


Taking the numerator of (209), collecting just those terms which contribute to the trace anomaly and employing Eq. (202), Eq. (209) becomes

$$
\begin{aligned}
& \tilde{T}_{\mu \nu \mu^{\prime} \nu^{\prime}}\left(k_{1}, k_{2}\right)=-\frac{i}{32} k_{1}^{\alpha} k_{2}^{\beta} \epsilon_{\nu \alpha \nu^{\prime} \beta} \int_{0}^{1} \mathrm{~d} x \int_{0}^{1-x} \mathrm{~d} y \int \frac{\mathrm{d}^{4} p}{(2 \pi)^{4}} \\
& \quad \times \int \frac{d^{n-4} \ell}{(2 \pi)^{n-4}} \frac{p^{2} \eta_{\mu \mu^{\prime}}+4 y(x+y-1) k_{1 \mu^{\prime}} k_{2 \mu}}{\left[p^{2}-\ell^{2}+2 k_{1} \cdot k_{2} y(1-y-x)\right]^{3}} \ell^{2} .
\end{aligned}
$$

To make sense of the integrals present in (210), we make a Wick rotation $k^{0} \rightarrow i k_{E}^{0}$ for any momentum $k^{\mu}$ : so, for instance, in the previous integral $p^{2} \rightarrow-p_{E}^{2}$, etc. So (210) is replaced by

$$
\begin{gathered}
\tilde{T}_{\mu \nu \mu^{\prime} \nu^{\prime}}\left(k_{1}, k_{2}\right)=\frac{1}{32} k_{1}^{\alpha} k_{2}^{\beta} \epsilon_{\nu \alpha \nu^{\prime} \beta} \int_{0}^{1} \mathrm{~d} x \int_{0}^{1-x} \mathrm{~d} y \int \frac{\mathrm{d}^{4} p}{(2 \pi)^{4}} \\
\times \int \frac{d^{n-4} \ell}{(2 \pi)^{n-4}} \frac{p^{2} \eta_{\mu \mu^{\prime}}-4 y(x+y-1) k_{1 \mu^{\prime}} k_{2 \mu}}{\left[p^{2}+\ell^{2}+2 k_{1} \cdot k_{2} y(1-y-x)\right]^{3}} \ell^{2},
\end{gathered}
$$

and we dispense from explicitly indicating the Euclidean momenta whenever it is not strictly necessary. Now the integrals are well defined and we can use the following results:

$$
\begin{aligned}
& \int \frac{d^{n-4} \ell}{(2 \pi)^{n-4}} \frac{\ell^{2}}{\left[p^{2}+2 k_{1} \cdot k_{2} y(1-y-x)+\ell^{2}\right]^{3}} \\
& =\frac{1}{(4 \pi)^{(n-4) / 2}} \frac{n-4}{4} \\
& \quad \times \frac{1}{\left[p^{2}+2 k_{1} \cdot k_{2} y(1-y-x)\right]^{4-\frac{n}{2}}} \Gamma\left(4-\frac{n}{2}\right), \\
& \int \frac{\mathrm{d}^{4} p}{(2 \pi)^{4}} \frac{1}{\left[p^{2}+2 k_{1} \cdot k_{2} y(1-y-x)\right]^{4-\frac{n}{2}}} \\
& =\frac{1}{(4 \pi)^{2}} \frac{\Gamma\left(2-\frac{n}{2}\right)}{\Gamma\left(4-\frac{n}{2}\right)}\left(\frac{1}{2 k_{1} \cdot k_{2} y(1-y-x)}\right)^{2-\frac{n}{2}}, \\
& \int \frac{\mathrm{d}^{4} p}{(2 \pi)^{4}} \frac{\left[p^{2}\right.}{\left[p^{2}+2 k_{1} \cdot k_{2} y(1-x-y)\right]^{4-\frac{n}{2}}} \\
& =\frac{2}{(4 \pi)^{2}} \frac{\Gamma\left(1-\frac{n}{2}\right)}{\Gamma\left(4-\frac{n}{2}\right)}\left(\frac{1}{2 k_{1} \cdot k_{2} y(1-y-x)}\right)^{1-\frac{n}{2}} .
\end{aligned}
$$

Using (212) and performing the integration over the Feynman parameters $(x, y)$ and returning to the Lorentzian metric, one obtains

$$
\begin{aligned}
& \tilde{T}_{\mu \nu \mu^{\prime} \nu^{\prime}}\left(k_{1}, k_{2}\right) \\
& \quad=\frac{1}{6144 \pi^{2}} k_{1}^{\alpha} k_{2}^{\beta} \epsilon_{\nu v^{\prime} \alpha \beta}\left(\eta_{\mu \mu^{\prime}} k_{1} \cdot k_{2}-k_{1 \mu^{\prime}} k_{2 \mu}\right) .
\end{aligned}
$$

Of course, as previously mentioned, one should symmetrize Eq. (213) with respect to $(\mu \leftrightarrow v)$ and $\left(\mu^{\prime} \leftrightarrow v^{\prime}\right)$. Then (213) becomes

$$
\begin{aligned}
& \tilde{T}_{\mu \nu \mu^{\prime} \nu^{\prime}}\left(k_{1}, k_{2}\right) \\
& \quad=\frac{1}{6144 \pi^{2}} k_{1}^{\alpha} k_{2}^{\beta}\left(k_{1} \cdot k_{2} t_{\mu \nu \mu^{\prime} \nu^{\prime} \alpha \beta}-t_{\mu \nu \mu^{\prime} \nu^{\prime} \alpha \beta}^{(21)}\right),
\end{aligned}
$$

The tensors $t$ and $t^{(21)}$ have been defined in (34) and (157).

On top of that one should add the contribution from the "cross diagram", namely, the contribution coming from the simultaneous exchanges $\left(k_{1} \leftrightarrow k_{2}, \mu \leftrightarrow \mu^{\prime}, v \leftrightarrow v^{\prime}\right)$. Hence, the sum of (214) with the cross diagram contribution gives rise to

$$
\begin{aligned}
& \tilde{T}_{\mu \nu \mu^{\prime} \nu^{\prime}}^{(\mathrm{tot})}\left(k_{1}, k_{2}\right) \\
& =\frac{1}{3072 \pi^{2}} k_{1}^{\alpha} k_{2}^{\beta}\left(k_{1} \cdot k_{2} t_{\mu \nu \mu^{\prime} \nu^{\prime} \alpha \beta}-t_{\mu \nu \mu^{\prime} \nu^{\prime} \alpha \beta}^{(21)}\right) .
\end{aligned}
$$

\section{B Derivation of Feynman rules}

\section{B.1 Ordinary gravity}

Consider a free theory coupled to ordinary gravity. We assume that the action has the expansion

$$
\begin{aligned}
S= & \sum_{n=0}^{\infty} S_{n} \equiv S_{0}+\sum_{n=1}^{\infty} \int \prod_{i=1}^{n} \mathrm{~d} x_{i} \\
& \times\left.\frac{1}{n !} \frac{\delta^{n} S}{\delta h_{\mu_{1} v_{1}}\left(x_{1}\right) \ldots \delta h_{\mu_{n} v_{n}}\left(x_{n}\right)}\right|_{h=0} \\
& \times h_{\mu_{1} v_{1}}\left(x_{1}\right) \ldots h_{\mu_{n} v_{n}}\left(x_{n}\right) \\
= & S_{0}+\left.\int \mathrm{d} x \frac{\delta S}{\delta h_{\mu \nu}(x)}\right|_{h=0} h_{\mu \nu}(x) \\
& +\left.\frac{1}{2} \int \mathrm{d} x_{1} \mathrm{~d} x_{2} \frac{\delta^{2} S}{\delta h_{\mu_{1} v_{1}}\left(x_{1}\right) \delta h_{\mu_{2} v_{2}}\left(x_{2}\right)}\right|_{h=0} \\
& \times h_{\mu_{1} v_{1}}\left(x_{1}\right) h_{\mu_{2} v_{2}}\left(x_{2}\right)+\cdots
\end{aligned}
$$

The e.m. tensor is defined as

$T^{\mu \nu}=\frac{2}{\sqrt{g}} \frac{\delta S}{\delta g_{\mu \nu}}, \quad T_{\mu \nu}=-\frac{2}{\sqrt{g}} \frac{\delta S}{\delta g^{\mu \nu}}$.

In the following we have in mind the free fermion theory in 4 defined by (23), and set $g_{\mu \nu}=\eta_{\mu \nu}+h_{\mu \nu}$.

We need the expansion

$$
\begin{aligned}
\sqrt{|g|}= & 1+\frac{1}{2}(\operatorname{tr} h)+\frac{1}{8}(\operatorname{tr} h)^{2}-\frac{1}{4}\left(\operatorname{tr} h^{2}\right) \\
& -\frac{1}{8}(\operatorname{tr} h)\left(\operatorname{tr} h^{2}\right)+\frac{1}{48}(\operatorname{tr} h)^{3}+\frac{1}{6}\left(\operatorname{tr} h^{3}\right)+\cdots \\
\equiv & \sum_{n=0} O_{n}(h), \\
\frac{1}{\sqrt{g}}= & 1-\frac{1}{2}(\operatorname{tr} h)+\frac{1}{8}(\operatorname{tr} h)^{2}+\frac{1}{4}\left(\operatorname{tr} h^{2}\right) \\
& -\frac{1}{8}(\operatorname{tr} h)\left(\operatorname{tr} h^{2}\right)-\frac{1}{48}(\operatorname{tr} h)^{3}-\frac{1}{6}\left(\operatorname{tr} h^{3}\right)+\cdots
\end{aligned}
$$




$$
\equiv \sum_{n=0} \hat{O}_{n}(h)
$$

where by $h$ is meant the matrix $h_{\mu \nu}$, and $O_{0}(h)=$ $1, O_{1}(h)=\frac{1}{2}(\operatorname{tr} h), \ldots$. Next we consider the complete expansion of (23) in powers of $h$, like (38). Now, using (217), one can write

$$
\begin{aligned}
T^{\mu \nu}(x)= & \frac{2}{\sqrt{g}}\left(\left.\frac{\delta S}{\delta h_{\mu \nu}(x)}\right|_{h=0}\right. \\
& +\left.\int \mathrm{d} x_{2} \frac{\delta^{2} S}{\delta h_{\mu v}(x) \delta h_{\mu_{2} \nu_{2}}\left(x_{2}\right)}\right|_{h=0} h_{\mu_{2} \nu_{2}}\left(x_{2}\right) \\
& +\frac{1}{2} \int \mathrm{d} x_{2} \mathrm{~d} x_{3} \\
& \times\left.\frac{\delta^{3} S}{\delta h_{\mu v}(x) \delta h_{\mu_{2} v_{2}}\left(x_{2}\right) \delta h_{\mu_{3} v_{3}}\left(x_{3}\right)}\right|_{h=0} \\
& \left.\times h_{\mu_{2} v_{2}}\left(x_{2}\right) h_{\mu_{3} v_{3}}\left(x_{3}\right)+\cdots\right) \\
\equiv & T_{(0)}^{\mu v}(x)+T_{(1)}^{\mu \nu}(x)+\cdots,
\end{aligned}
$$

which implies

$$
\begin{aligned}
T_{(n)}^{\mu \nu}(x)= & \sum_{m=0}^{n} \hat{O}_{n-m}(h(x)) \frac{2}{m !} \\
& \left.\cdot \int \prod_{i=1}^{m} \mathrm{~d} x_{i} \frac{\delta^{m+1} S}{\delta h_{\mu v}(x) \delta h_{\mu_{1} v_{1}}\left(x_{1}\right) \ldots \delta h_{\mu_{m} v_{m}}\left(x_{m}\right)}\right|_{h=0} \\
& \times h_{\mu_{1} v_{1}}\left(x_{1}\right) \ldots h_{\mu_{m} v_{m}}\left(x_{m}\right) .
\end{aligned}
$$

So we can rewrite

$$
S_{n}=\frac{1}{2 n} \int \mathrm{d} x\left(\sum_{m=1}^{n} O_{n-m}(h(x)) T_{(m-1)}^{\mu \nu}(x)\right) h_{\mu \nu}(x) .
$$

For instance

$$
\begin{aligned}
S_{1}= & \frac{1}{2} \int \mathrm{d} x T_{(0)}^{\mu \nu}(x) h_{\mu \nu}(x), \\
S_{2}= & \frac{1}{4} \int \mathrm{d} x\left(T_{(1)}^{\mu \nu}(x)+\frac{1}{2}(\operatorname{tr} h(x)) T_{(0)}^{\mu \nu}(x)\right) h_{\mu v}(x), \\
S_{3}= & \frac{1}{6} \int \mathrm{d} x\left(T_{(2)}^{\mu \nu}(x)+\frac{1}{2}(\operatorname{tr} h(x)) T_{(1)}^{\mu \nu}(x)\right. \\
& \left.+\frac{1}{8}\left((\operatorname{tr} h(x))^{2}-2\left(\operatorname{tr} h^{2}(x)\right)\right) T_{(0)}^{\mu \nu}(x)\right) h_{\mu \nu}(x),
\end{aligned}
$$

and

$$
\begin{aligned}
& T_{(0)}^{\mu \nu}(x)=\left.2 \frac{\delta S}{\delta h_{\mu \nu}(x)}\right|_{h=0}, \\
& T_{(1)}^{\mu v}(x)=-\left.(\operatorname{tr} h(x)) \frac{\delta S}{\delta h_{\mu v}(x)}\right|_{h=0} \\
& +\left.2 \int \mathrm{d} x_{1} \frac{\delta^{2} S}{\delta h_{\mu \nu}(x) \delta h_{\mu_{1} v_{1}}\left(x_{1}\right)}\right|_{h=0} h_{\mu_{1} v_{1}}\left(x_{1}\right), \\
& T_{(2)}^{\mu \nu}(x)=\left.\frac{1}{4}\left((\operatorname{tr} h(x))^{2}+2\left(\operatorname{tr} h(x)^{2}\right)\right) \frac{\delta S}{\delta h_{\mu \nu}(x)}\right|_{h=0} \\
& -\left.(\operatorname{tr} h(x)) \int \mathrm{d} x_{1} \frac{\delta^{2} S}{\delta h_{\mu v}(x) \delta h_{\mu_{1} v_{1}}\left(x_{1}\right)}\right|_{h=0} h_{\mu_{1} v_{1}}\left(x_{1}\right) \\
& +\left.2 \int \mathrm{d} x_{1} \mathrm{~d} x_{2} \frac{\delta^{3} S}{\delta h_{\mu v}(x) \delta h_{\mu_{1} \nu_{1}}\left(x_{1}\right) \delta h_{\mu_{2} \nu_{2}}\left(x_{2}\right)}\right|_{h=0} \\
& \times h_{\mu_{1} v_{1}}\left(x_{1}\right) h_{\mu_{2} v_{2}}\left(x_{2}\right) \text {. }
\end{aligned}
$$

Remark Since $S=\int \sqrt{|g|} \mathcal{L}$, the derivatives of $S$ in the previous formulas, when applied to $\sqrt{|g|}$, will produce terms $\sim \mathcal{L}$ which vanish on shell. These are contact terms. They produce contraction of the Feynman diagrams whereby a fermion internal line drops and the two endpoints collapse to a single one. These are contact terms. They are not the only ones. Other contact terms are produced by seagull vertices, i.e. vertices with two fermion legs and two or more graviton legs, by contracting the fermion legs with a propagator, thus forming a fermion loop.

\section{B.2 One-loop one-point function}

Representing by $\phi$ the matter fields in the model, the oneloop one-point function of $T^{\mu v}$ in the presence of a metric $g_{\mu \nu}=\eta_{\mu \nu}+h_{\mu \nu}$ is

$$
\begin{aligned}
\left.\left\langle T^{\mu \nu}(x)\right\rangle\right\rangle= & \int \mathcal{D} \phi T^{\mu \nu}(x) e^{i S[\phi, h]} \\
= & \int \mathcal{D} \phi\left(T_{(0)}^{\mu \nu}(x)+T_{(1)}^{\mu \nu}(x)+T_{(2)}^{\mu \nu}(x)+\cdots\right) \\
& \times e^{i\left(S_{0}+S_{1}+S_{2}+\cdots\right)} \\
= & \int \mathcal{D} \phi\left[\left(T_{(0)}^{\mu \nu}(x)+T_{(1)}^{\mu \nu}(x)+T_{(2)}^{\mu \nu}(x)+\cdots\right)\right. \\
& \left.\times e^{i\left(S_{1}+S_{2}+\cdots\right)}\right] e^{i S_{0}} .
\end{aligned}
$$

$e^{i S_{0}}$ has been singled out as the free part of the integration measure. The rest of $S$ (the interaction) is treated perturbatively.

Rearranging (229) order by order in $h$ :

$$
\begin{aligned}
& \left\langle T^{\mu \nu}(x)\right\rangle=\int \mathcal{D} \phi T_{(0)}^{\mu \nu}(x) e^{i S_{0}} \\
& +\int \mathcal{D} \phi\left(i S_{1} T_{(0)}^{\mu \nu}(x)+T_{(1)}^{\mu \nu}(x)\right) e^{i S_{0}} \\
& +\int \mathcal{D} \phi\left(\left(i S_{2}-\frac{1}{2} S_{1}^{2}\right) T_{(0)}^{\mu v}(x)+i S_{1} T_{(1)}^{\mu v}(x)+T_{(2)}^{\mu v}(x)\right) e^{i S_{0}}
\end{aligned}
$$




$$
\begin{aligned}
& +\int \mathcal{D} \phi\left(\left(i S_{3}-S_{1} S_{2}-\frac{i}{3 !} S_{1}^{3}\right) T_{(0)}^{\mu v}(x)+\left(i S_{2}-\frac{1}{2} S_{1}^{2}\right) T_{(1)}^{\mu v}(x)\right. \\
& \left.+i S_{1} T_{(2)}^{\mu \nu}(x)+T_{(3)}^{\mu \nu}(x)\right) e^{i S_{0}}+\cdots
\end{aligned}
$$

Next we introduce auxiliary external currents and couple them to the free field in $S_{0}$. For instance if the free fields are $\psi, \bar{\psi}$, we introduce $j, \bar{j}$ and add a term

$$
\begin{aligned}
\left\langle\left\langle T^{\mu v}(x)\right\rangle\right\rangle[j, \bar{j}]= & \int \mathcal{D} \bar{\psi} \mathcal{D} \psi(\ldots \ldots) \exp \left[i S_{0}\right. \\
& \left.+i \int(\bar{j} \psi+\bar{\psi} j)\right]
\end{aligned}
$$

and set at the end $j=\bar{j}=0$. At this point in $(\ldots \ldots \ldots)$ one can replace $\psi$ by $\frac{\delta}{\delta \bar{j}}$ and $\bar{\psi}$ by $-\frac{\delta}{\delta j}$, so that the only remaining dependence on $\psi$ and $\bar{\psi}$ is in the factor $\exp \left[i S_{0}+\right.$ $\left.\int(\bar{j} \psi+\bar{\psi} j)\right]$. Since the exponent is a quadratic expression, one can formally integrate over $\psi$ and $\bar{\psi}$ by completing the square. This leads to an irrelevant infinite constant times

$$
\exp \left[-i \int \bar{j} P j\right]
$$

where $P$ is the inverse of the kinetic differential operator in $S_{0}$, i.e. the propagator in configuration space. Finally

$$
\begin{aligned}
\left\langle\left\langle T^{\mu \nu}(x)\right\rangle\right\rangle= & T_{(0)}^{\mu \nu}(x) \exp \left[-i \int \bar{j} P j\right] \\
& +\left(i S_{1} T_{(0)}^{\mu \nu}(x)+T_{(1)}^{\mu \nu}(x)\right) \exp \left[-i \int \bar{j} P j\right] \\
& +\left(\left(i S_{2}-\frac{1}{2} S_{1}^{2}\right) T_{(0)}^{\mu \nu}(x)\right. \\
& \left.+i S_{1} T_{(1)}^{\mu \nu}(x)+T_{(2)}^{\mu \nu}(x)\right) \exp \left[-i \int \bar{j} P j\right] \\
& +\left(\left(i S_{3}-S_{1} S_{2}-\frac{i}{3 !} S_{1}^{3}\right) T_{(0)}^{\mu \nu}(x)\right. \\
& \left.+\left(i S_{2}-\frac{1}{2} S_{1}^{2}\right) T_{(1)}^{\mu \nu}(x)+i S_{1} T_{(2)}^{\mu \nu}(x)+T_{(3)}^{\mu \nu}(x)\right) \\
& \left.\cdot \exp \left[-i \int \bar{j} P j\right]\right]\left.\right|_{j=\bar{j}=0}+\cdots
\end{aligned}
$$

where all the $\psi, \bar{\psi}$ fields in $T_{(n)}, S_{n}$ are understood to be replaced by $\frac{\delta}{\delta \bar{j}}$ and $-\frac{\delta}{\delta j}$, respectively. This is the final expression of the 1pt one-loop correlator from which the Feynman rules are extracted. Equation (232) is thus rewritten as

$$
\begin{aligned}
& \left\langle\left\langle T^{\mu \nu}(x)\right\rangle\right\rangle=\left\langle 0\left|T_{(0)}^{\mu \nu}(x)\right| 0\right\rangle \\
& +\left\langle 0\left|\mathcal{T}\left(i S_{1} T_{(0)}^{\mu \nu}(x)+T_{(1)}^{\mu \nu}(x)\right)\right| 0\right\rangle \\
& +\left\langle 0\left|\mathcal{T}\left(\left(i S_{2}-\frac{1}{2} S_{1}^{2}\right) T_{(0)}^{\mu \nu}(x)+i S_{1} T_{(1)}^{\mu v}(x)+T_{(2)}^{\mu \nu}(x)\right)\right| 0\right\rangle \\
& \quad+\langle 0| \mathcal{T}\left(\left(i S_{3}-S_{1} S_{2}-\frac{i}{3 !} S_{1}^{3}\right) T_{(0)}^{\mu v}(x)+\left(i S_{2}-\frac{1}{2} S_{1}^{2}\right) T_{(1)}^{\mu v}(x)\right.
\end{aligned}
$$

$$
\begin{aligned}
& \left.+i S_{1} T_{(2)}^{\mu \nu}(x)+T_{(3)}^{\mu \nu}(x)\right)|0\rangle \\
& +\cdots
\end{aligned}
$$

and the time-ordered amplitudes are computed by means of Feynman diagrams.

\section{B.3 MAT background}

In this subsection the reference is to the expanded action (137). We rewrite it as

$$
\begin{aligned}
S= & S_{0}+\sum_{n+m \geq 1} \frac{1}{n !} \frac{1}{m !} \int \prod_{i=0}^{n} \prod_{j=0}^{m} \mathrm{~d} x_{i} \mathrm{~d} y_{j} \\
& \times\left.\frac{\delta^{i+j} S}{\delta h_{\mu_{1} v_{1}}\left(x_{1}\right) \ldots \delta h_{\mu_{i} v_{i}}\left(x_{i}\right) \delta k_{\lambda_{1} \rho_{1}}(1) \ldots \delta k_{\lambda_{j} \rho_{j}\left(y_{j}\right)}}\right|_{h, k=0} \\
& \times h_{\mu_{1} v_{1}}\left(x_{1}\right) \ldots h_{\mu_{j} v_{j}}\left(x_{j}\right) k_{\lambda_{1} \rho_{1}}(1) \ldots k_{\lambda_{j} \rho_{j}}\left(y_{j}\right) \\
= & S_{0}+\left.\int \mathrm{d} x \frac{\delta S}{\delta h_{\mu \nu}(x)}\right|_{h, k=0} h_{\mu \nu}(x) \\
& +\left.\int \mathrm{d} y \frac{\delta S}{\delta k_{\lambda \rho}(x)}\right|_{h, k=0} k_{\lambda \rho}(y) \\
& +\left.\frac{1}{2} \int \mathrm{d} x_{1} \mathrm{~d} x_{2} \frac{\delta^{2} S}{\delta h_{\mu_{1} \nu_{1}}\left(x_{1}\right) \delta h_{\mu_{2} \nu_{2}}\left(x_{2}\right)}\right|_{h, k=0} \\
& \times h_{\mu_{1} v_{1}}\left(x_{1}\right) h_{\mu_{2} v_{2}}\left(x_{2}\right)+\ldots \\
\equiv & \sum_{n, m=0}^{\infty} S_{n, m}
\end{aligned}
$$

where $S_{0} \equiv S_{0,0}$. As long as we differentiate $S$ from the right it functionally depends on the axial-complex variable $g+\gamma_{5} f$. So the functional derivatives with respect to $h_{\mu \nu}$ and $k_{\mu \nu}$ have to be understood as

$$
\begin{gathered}
\frac{\delta}{\delta h_{\mu \nu}(x)}=\int \mathrm{d}^{4} x^{\prime} \frac{\delta G_{\lambda \rho}\left(x^{\prime}\right)}{\delta h_{\mu \nu}(x)} \frac{\vec{\delta}}{\delta G_{\lambda \rho}\left(x^{\prime}\right)}=\frac{\vec{\delta}}{\delta G_{\mu \nu}(x)}, \\
\frac{\delta}{\delta k_{\mu \nu}(x)}=\int \mathrm{d}^{4} x^{\prime} \frac{\delta G_{\lambda \rho}\left(x^{\prime}\right)}{\delta k_{\mu \nu}(x)} \frac{\vec{\delta}}{G_{\lambda \rho}\left(x^{\prime}\right)}=\gamma_{5} \frac{\vec{\delta}}{\delta G_{\mu \nu}(x)} .
\end{gathered}
$$

Now, going back to the definitions of $T^{\mu \nu}$ and $T_{5}^{\mu \nu},(123)$, (125) and (126), one can see that, in the case when $\sqrt{|G|}$ is absorbed in $\psi$ we can write

$$
\begin{aligned}
T^{\mu \nu}(x)= & 2\left(\left.\frac{\delta S}{\delta h_{\mu \nu}(x)}\right|_{h, k=0}\right. \\
& +\left.\int \mathrm{d} x_{2} \frac{\delta^{2} S}{\delta h_{\mu \nu}(x) \delta h_{\mu_{2} \nu_{2}}\left(x_{2}\right)}\right|_{h, k=0} h_{\mu_{2} v_{2}}\left(x_{2}\right) \\
& \left.+\left.\int \mathrm{d} y \frac{\delta^{2} S}{\delta h_{\mu \nu}(x) \delta k_{\lambda \rho}(y)}\right|_{h, k=0} k_{\lambda \rho}(y)+\cdots\right) \\
& =T_{(0,0)}^{\mu \nu}(x)+T_{(1,0)}^{\mu \nu}(x)+T_{(0,1)}^{\mu \nu}(x)+\cdots
\end{aligned}
$$


and

$$
\begin{aligned}
T_{5}^{\mu \nu}(x)= & 2\left(\left.\frac{\delta S}{\delta k_{\mu \nu}(x)}\right|_{h, k=0}\right. \\
& +\left.\int \mathrm{d} y_{2} \frac{\delta^{2} S}{\delta k_{\mu \nu}(x) \delta k_{\mu_{2} v_{2}}\left(y_{2}\right)}\right|_{h, k=0} k_{\mu_{2} v_{2}}\left(y_{2}\right) \\
& \left.+\left.\int \mathrm{d} y \frac{\delta^{2} S}{\delta k_{\mu \nu}(x) \delta h_{\lambda \rho}(y)}\right|_{h, k=0} h_{\lambda \rho}(y)+\cdots\right) \\
= & T_{5(0,0)}^{\mu \nu}(x)+T_{5(0,1)}^{\mu \nu}(x)+T_{5(1,0)}^{\mu \nu}(x)+\cdots .
\end{aligned}
$$

Therefore

$$
\begin{aligned}
& T_{(n, m)}^{\mu v}(x)=\sum_{i=0}^{n} \sum_{j=0}^{m} \frac{2}{i ! j !} \int \prod_{i=1}^{n} \prod_{j=1}^{m} \mathrm{~d} x_{i} \mathrm{~d} y_{j} \\
& \quad \times\left.\frac{\delta^{i+j+1} S}{\delta h_{\mu v}(x) \delta h_{\mu \nu_{1} \nu_{1}}\left(x_{1}\right) \ldots \delta h_{\mu_{i} \nu_{i}}\left(x_{i}\right) \delta k_{\lambda_{1} \rho_{1}}(1) \ldots \delta k_{\lambda_{j} \rho_{j}}\left(y_{j}\right)}\right|_{h, k=0} \\
& \quad \times h_{\mu_{1} \nu_{1}}\left(x_{1}\right) \ldots h_{\mu_{j} \nu_{j}}\left(x_{j}\right) k_{\lambda_{1} \rho_{1}}(1) \ldots k_{\lambda_{j} \rho_{j}}\left(y_{j}\right)
\end{aligned}
$$

and

$$
\begin{aligned}
& T_{5(n, m)}^{\lambda \rho}(x)=\sum_{i=0}^{n} \sum_{j=0}^{m} \frac{2}{i ! j !} \int \prod_{i=1}^{n} \prod_{j=1}^{m} \mathrm{~d} x_{i} \mathrm{~d} y_{j} \\
& \quad \times\left.\frac{\delta^{i+j+1} S}{\delta h_{\mu_{1} \nu_{1}}\left(x_{1}\right) \cdots \delta h_{\mu_{i} \nu_{i}}\left(x_{i}\right) \delta k_{\lambda \rho}(x) \delta k_{\lambda_{1} \rho_{1}}(1) \cdots \delta k_{\lambda_{j} \rho_{j}}\left(y_{j}\right)}\right|_{h, k=0} \\
& \quad \times h_{\mu_{1} \nu_{1}}\left(x_{1}\right) \cdots h_{\mu_{j} v_{j}}\left(x_{j}\right) k_{\lambda_{1} \rho_{1}}(1) \cdots k_{\lambda_{j} \rho_{j}}\left(y_{j}\right) .
\end{aligned}
$$

So,

$$
\begin{aligned}
S_{n, m}= & c_{n, m}\left(\frac{1}{2 n} \sum_{i=0}^{n} \sum_{j=0}^{m} \int \mathrm{d} x T_{(i-1, j)}^{\mu \nu}(x) h_{\mu v}(x)\right. \\
& \left.+\frac{1}{2 m} \sum_{i=0}^{n} \sum_{j=0}^{m} \int \mathrm{d} x T_{5(i, j-1)}^{\mu \nu}(x) k_{\mu \nu}(x)\right)
\end{aligned}
$$

where $c_{n, m}=1$ for either $n=0$ or $m=0, c_{n, m}=\frac{1}{2}$ otherwise. For instance

$$
\begin{aligned}
& S_{1,0}=\frac{1}{2} \int \mathrm{d} x T_{(0,0)}^{\mu v}(x) h_{\mu \nu}(x)=\left.\int \mathrm{d} x \frac{\delta S}{\delta h_{\mu \nu}(x)}\right|_{h, k=0} h_{\mu v}(x) \\
& S_{0,1}=\frac{1}{2} \int \mathrm{d} y T_{5(0,0)}^{\mu v}(y) k_{\mu \nu}(y)=\left.\int \mathrm{d} y \frac{\delta S}{\delta k_{\mu v}(y)}\right|_{h, k=0} k_{\mu \nu}(y) \\
& S_{2,0}=\frac{1}{8} \int \mathrm{d} x T_{(1,0)}^{\mu \nu}(x) h_{\mu v}(x) \\
& S_{0,2}=\frac{1}{8} \int \mathrm{d} y T_{5(0,1)}^{\mu v}(y) k_{\mu \nu}(y) \\
& S_{1,1}=\frac{1}{4} \int \mathrm{d} x T_{(0,1)}^{\mu v}(x) h_{\mu \nu}(x)+\frac{1}{4} \int \mathrm{d} y T_{5(1,0)}^{\mu \nu}(y) k_{\mu \nu}(y)
\end{aligned}
$$

and

$$
\begin{aligned}
T_{(0,0)}^{\mu \nu}(x)= & \left.2 \frac{\delta S}{\delta h_{\mu \nu}(x)}\right|_{h, k-=0}, \\
T_{(1,0)}^{\mu \nu}(x)= & \left.2 \int \mathrm{d} x_{1} \frac{\delta^{2} S}{\delta h_{\mu v}(x) \delta h_{\mu_{1} v_{1}}\left(x_{1}\right)}\right|_{h, k=0} \\
& \times h_{\mu_{1} v_{1}}\left(x_{1}\right), \\
T_{(0,1)}^{\mu \nu}(x)= & \left.2 \int \mathrm{d} y \frac{\delta^{2} S}{\delta h_{\mu \nu}(x) \delta k_{\lambda \rho}(y)}\right|_{h, k=0} \\
& \times k_{\lambda \rho}(y),
\end{aligned}
$$

$$
\begin{aligned}
T_{(2,0)}^{\mu \nu}(x)= & \left.\int \mathrm{d} x_{1} \mathrm{~d} x_{2} \frac{\delta^{3} S}{\delta h_{\mu \nu}(x) \delta h_{\mu_{1} \nu_{1}}\left(x_{1}\right) \delta h_{\mu_{2} \nu_{2}}\left(x_{2}\right)}\right|_{h, k=0} \\
& \times h_{\mu_{1} \nu_{1}}\left(x_{1}\right) h_{\mu_{2} v_{2}}\left(x_{2}\right), \\
T_{(0,2)}^{\mu \nu}(x)= & \left.\int \mathrm{d} y_{1} \mathrm{~d} y_{2} \frac{\delta^{3} S}{\delta h_{\mu \nu}(x) \delta k_{\lambda_{1} \rho_{1}}(1) \delta k_{\lambda_{2} \rho_{2}}\left(y_{2}\right)}\right|_{h, k=0} \\
& \times k_{\lambda_{1} \rho_{1}}(1) k_{\lambda_{2} \rho_{2}}\left(y_{2}\right), \\
T_{(1,1)}^{\mu \nu}(x)= & \left.2 \int \mathrm{d} x_{1} \mathrm{~d} y \frac{\delta^{3} S}{\delta h_{\mu \nu}(x) \delta h_{\mu_{1} \nu_{1}}\left(x_{1}\right) \delta k_{\lambda \rho}(y)}\right|_{h, k=0} \\
& \times k_{\mu_{1} \nu_{1}}\left(x_{1}\right) k_{\lambda \rho}(y) .
\end{aligned}
$$

\section{Similarly}

$$
\begin{aligned}
& T_{5(0,0)}^{\mu \nu}(x)=\left.2 \frac{\delta S}{\delta k_{\mu \nu}(x)}\right|_{h, k-=0}, \\
& T_{5(1,0)}^{\mu \nu}(x)=\left.2 \int \mathrm{d} x_{1} \frac{\delta^{2} S}{\delta k_{\mu \nu}(x) \delta h_{\mu_{1} \nu_{1}}\left(x_{1}\right)}\right|_{h, k=0} h_{\mu_{1} \nu_{1}}\left(x_{1}\right)
\end{aligned}
$$$$
T_{5(0,1)}^{\mu \nu}(x)=\left.2 \int \mathrm{d} y \frac{\delta^{2} S}{\delta k_{\mu \nu}(x) \delta k_{\lambda \rho}(y)}\right|_{h, k=0} k_{\lambda \rho}(y),
$$

$$
\begin{aligned}
T_{5(2,0)}^{\mu \nu}(x)= & \left.\int \mathrm{d} x_{1} \mathrm{~d} x_{2} \frac{\delta^{3} S}{\delta k_{\mu \nu}(x) \delta h_{\mu_{1} v_{1}}\left(x_{1}\right) \delta h_{\mu_{2} v_{2}}\left(x_{2}\right)}\right|_{h, k=0} \\
& \times h_{\mu_{1} v_{1}}\left(x_{1}\right) h_{\mu_{2} v_{2}}\left(x_{2}\right),
\end{aligned}
$$

$$
\begin{aligned}
T_{5(0,2)}^{\mu \nu}(x)= & \left.\int \mathrm{d} y_{1} \mathrm{~d} y_{2} \frac{\delta^{3} S}{\delta k_{\mu v}(x) \delta k_{\lambda_{1} \rho_{1}}(1) \delta k_{\lambda_{2} \rho_{2}}\left(y_{2}\right)}\right|_{h, k=0} \\
& \times k_{\lambda_{1} \rho_{1}(1) k_{\lambda_{2} \rho_{2}}\left(y_{2}\right),} \\
T_{5(1,1)}^{\mu \nu}(x)= & \left.2 \int \mathrm{d} x_{1} \mathrm{~d} y \frac{\delta^{3} S}{\delta k_{\mu \nu}(x) \delta h_{\mu_{1} \nu_{1}}\left(x_{1}\right) \delta k_{\lambda \rho}(y)}\right|_{h, k=0} \\
& \times h_{\mu_{1} \nu_{1}}\left(x_{1}\right) k_{\lambda \rho}(y) .
\end{aligned}
$$

The explicit expression of $T_{(0,0)}^{\mu \nu}(x)$ and $T_{5(0,0)}^{\mu \nu}(x)$ are given in Eqs. (148) and (149). 


\section{B.4 The one-loop one-point functions}

The one-loop one-point functions of $T^{\mu \nu}$ and $T_{5}^{\mu \nu}$ are defined in path integral terms as follows:

$$
\begin{aligned}
\left\langle\left\langle\mathrm{T}^{\mu v}(x)\right\rangle\right\rangle= & \int \mathcal{D} \phi \mathrm{T}^{\mu \nu}(x) e^{i S[\phi, h]} \\
= & \int \mathcal{D} \phi\left[\left(\mathrm{T}_{(0,0)}^{\mu v}(x)+\mathrm{T}_{(1,0)}^{\mu \nu}(x)\right.\right. \\
& \left.\left.+\mathrm{T}_{(0,1)}^{\mu \nu}(x)+\cdots\right) e^{i\left(S_{10}+S_{01}+\cdots\right)}\right] e^{i S_{0}}
\end{aligned}
$$

where $\mathrm{T}$ can be either $T$ or $T_{5}$. Expanding the exponential:

$$
\begin{aligned}
\left\langle\left\langle\mathrm{T}^{\mu \nu}(x)\right\rangle\right\rangle= & \int \mathcal{D} \phi \mathrm{T}_{(0,0)}^{\mu \nu}(x) e^{i S_{0}} \\
& +\int \mathcal{D} \phi\left(i S_{10} \mathrm{~T}_{(0,0)}^{\mu \nu}(x)+\mathrm{T}_{(1,0)}^{\mu \nu}(x)\right) e^{i S_{0}} \\
& +\int \mathcal{D} \phi\left(i S_{01} \mathrm{~T}_{(0,0)}^{\mu \nu}(x)+\mathrm{T}_{(0,1)}^{\mu \nu}(x)\right) e^{i S_{0}} \\
& +\int \mathcal{D} \phi\left(\left(i S_{20}-\frac{1}{2} S_{10}^{2}\right) \mathrm{T}_{(0,0)}^{\mu \nu}(x)\right. \\
& \left.+i S_{10} \mathrm{~T}_{(1,0)}^{\mu \nu}(x)+\mathrm{T}_{(2,0)}^{\mu \nu}(x)\right) e^{i S_{0}} \\
& +\int \mathcal{D} \phi\left(\left(i S_{02}-\frac{1}{2} S_{01}^{2}\right) \mathrm{T}_{(0,0)}^{\mu \nu}(x)\right. \\
& \left.+i S_{01} \mathrm{~T}_{(0,1)}^{\mu \nu}(x)+\mathrm{T}_{(0,2)}^{\mu \nu}(x)\right) e^{i S_{0}} \\
& +\int \mathcal{D} \phi\left(\left(i S_{11}-S_{01} S_{01}\right) \mathrm{T}_{(0,0)}^{\mu \nu}(x)\right. \\
& +i S_{01} \mathrm{~T}_{(1,0)}^{\mu \nu}(x) \\
& \left.+i S_{10} \mathrm{~T}_{(0,1)}^{\mu \nu}(x)+\mathrm{T}_{(1,1)}^{\mu \nu}(x)\right) e^{i S_{0}} \\
& +\cdots
\end{aligned}
$$

Next we introduce auxiliary external currents $J$ and $\bar{J}$ and couple them to the free field $\bar{\Psi}, \Psi$ in $S_{0}$.

$$
\begin{aligned}
\left\langle\left\langle T^{\mu \nu}(x)\right\rangle\right\rangle[J, \bar{J}]= & \int \mathcal{D} \bar{\Psi} \mathcal{D} \Psi(\ldots \ldots \ldots) \\
& \times \exp \left[i S_{0}+i \int(\bar{J} \Psi+\bar{\Psi} J)\right]
\end{aligned}
$$

and set at the end $J=\bar{J}=0$. At this point in $(\ldots \ldots \ldots)$ one can replace $\Psi$ by $\frac{\delta}{\delta \bar{J}}$ and $\bar{\Psi}$ by $-\frac{\delta}{\delta J}$, so that the only remaining dependence on $\Psi$ and $\bar{\Psi}$ is in the factor $\exp \left[i S_{0}+\right.$ $\left.\int(\bar{J} \Psi+\bar{\Psi} J)\right]$. Formally integrating over $\Psi$ and $\bar{\Psi}$ leads to an irrelevant infinite constant times

$\exp \left[-i \int \bar{J} P J\right]$

where $P$ is the inverse of the kinetic differential operator in $S_{0}$, i.e. the propagator in configuration space. The final expression is the same as (260) with $e^{i S_{0}}$ replaced by $\exp \left[-i \int \bar{j} P j\right]$, from which the Feynman rules can be extracted. This is interpreted as

$$
\begin{aligned}
& \left\langle\left\langle\mathrm{T}^{\mu \nu}(x)\right\rangle\right\rangle=\left\langle 0\left|\mathrm{~T}_{(0,0)}^{\mu \nu}(x)\right| 0\right\rangle \\
& +\left\langle 0\left|\mathcal{T}\left(i S_{10} \mathrm{~T}_{(0,0)}^{\mu \nu}(x)+\mathrm{T}_{(1,0)}^{\mu \nu}(x)\right)\right| 0\right\rangle \\
& +\left\langle 0\left|\mathcal{T}\left(i S_{01} \mathrm{~T}_{(0,0)}^{\mu \nu}(x)+\mathrm{T}_{(0,1)}^{\mu \nu}(x)\right)\right| 0\right\rangle \\
& +\langle 0| \mathcal{T}\left(\left(i S_{20}-\frac{1}{2} S_{10}^{2}\right) \mathrm{T}_{(0,0)}^{\mu \nu}(x)\right. \\
& \left.+i S_{10} \mathrm{~T}_{(1,0)}^{\mu \nu}(x)+\mathrm{T}_{(2,0)}^{\mu \nu}(x)\right)|0\rangle \\
& +\langle 0| \mathcal{T}\left(\left(i S_{02}-\frac{1}{2} S_{01}^{2}\right) \mathrm{T}_{(0,0)}^{\mu \nu}(x)\right. \\
& \left.+i S_{01} \mathrm{~T}_{(0,1)}^{\mu \nu}(x)+\mathrm{T}_{(0,2)}^{\mu \nu}(x)\right)|0\rangle \\
& +\langle 0| \mathcal{T} m\left(\left(i S_{11}-S_{01} S_{01}\right) \mathrm{T}_{(0,0)}^{\mu \nu}(x)+i S_{01} \mathrm{~T}_{(1,0)}^{\mu \nu}(x)\right. \\
& \left.+i S_{10} \mathrm{~T}_{(0,1)}^{\mu \nu}(x)+\mathrm{T}_{(1,1)}^{\mu \nu}(x)\right)|0\rangle \\
& +\cdots \\
& \equiv\left\langle 0\left|\mathrm{~T}_{(0,0)}^{\mu \nu}(x)\right| 0\right\rangle \\
& +\sum_{n+m \geq 1}^{\infty} \frac{1}{2^{n+m} n ! m !} \int \prod_{i, j, i+j \geq 1} \mathrm{~d} x_{i} \mathrm{~d} y_{j} h_{\mu_{1} \nu_{1}}\left(x_{1}\right) \cdots \\
& \times h_{\mu_{i} v_{i}}\left(x_{i}\right) k_{\lambda_{1} \rho_{1}}(1) \ldots k_{\lambda_{j} \rho_{j}}\left(y_{j}\right) \\
& \times \mathbf{T}^{\mu \nu \mu_{1} v_{1} \ldots \mu_{n} v_{n}, \lambda_{1} \rho_{1} \cdots \lambda_{m} \rho_{m}}\left(x, x_{1}, \ldots, x_{n}, 1, \ldots, y_{m}\right) \text {. }
\end{aligned}
$$

The expansion coefficients $\mathbf{T}^{\mu \nu \mu_{1} v_{1} \cdots \mu_{n} v_{n}}\left(x, x_{1}, \cdots, x_{n}\right)$, where $\mathbf{T}$ stands both for $\mathcal{T}$ and $\mathcal{T}_{5}$, are introduced for convenience.

\section{B.5 Trace Ward indentities}

The quantum Ward identities for the Weyl and axial Weyl symmetry are given by (150) and (151). We need to expand them in series of $h$ and $k$. With reference to (264) we get

$$
\begin{aligned}
\mathcal{T}_{(0,0)}(x) \equiv & \left\langle 0\left|T_{(0,0)}{ }_{\mu}^{\mu}(x)\right| 0\right\rangle=0, \\
\mathcal{T}_{(1,0)}\left(x, x_{1}\right) \equiv & \mathcal{T}_{(1,0) \mu}^{\mu \mu_{1} v_{1}}\left(x, x_{1}\right) \\
& +2 \delta\left(x-x_{1}\right)\left\langle 0\left|T_{(0,0)}^{\mu_{1} v_{1}}\left(x_{1}\right)\right| 0\right\rangle=0,
\end{aligned}
$$

$$
\begin{aligned}
\mathcal{T}_{(0,1)}\left(x, y_{1}\right) \equiv & \mathcal{T}_{(0,1)} \underset{\mu}{\mu \mu_{1} v_{1}}\left(x, y_{1}\right) \\
& +2 \delta\left(x-y_{1}\right)\left\langle 0\left|T_{5(0,0)}^{\mu_{1} v_{1}}\left(y_{1}\right)\right| 0\right\rangle=0
\end{aligned}
$$

$$
\begin{aligned}
\mathcal{T}_{(1,1)}\left(x, x_{1}, y_{1}\right) \equiv & \mathcal{T}_{(1,1)} \underset{\mu}{\mu \mu_{1} v_{1} \lambda_{1} \rho_{1}}\left(x, x_{1}, y_{1}\right) \\
& +2 \delta\left(x-x_{1}\right) \mathcal{T}_{(0,1)}^{\mu_{1} v_{1} \lambda_{1} \rho_{1}}\left(x_{1}, y_{1}\right) \\
& +2 \delta\left(x-y_{1}\right) \mathcal{T}_{5(1,0)}^{\mu_{1} v_{1} \lambda_{1} \rho_{1}}\left(x_{1}, y_{1}\right)=0,
\end{aligned}
$$

$$
\begin{aligned}
\mathcal{T}_{(2,0)}\left(x, x_{1}, x_{2}\right) \equiv & \mathcal{T}_{(2,0)} \mu_{\mu}^{\mu \mu_{1} v_{1} \mu_{2} v_{2}}\left(x, x_{1}, x_{2}\right) \\
& +2\left(\delta\left(x-x_{1}\right)\right.
\end{aligned}
$$




$$
\left.+\delta\left(x-x_{2}\right)\right) \mathcal{T}_{(1,0)}^{\mu_{1} v_{1} \mu_{2} v_{2}}\left(x_{1}, x_{2}\right)=0,
$$

$\mathcal{T}_{(0,2)}\left(x, y_{1}, y_{2}\right) \equiv \mathcal{T}_{(0,2)} \mu_{\mu}^{\mu \lambda_{1} \rho_{1} \lambda_{2} \rho_{2}}\left(x, y_{1}, y_{2}\right)$

$$
\begin{aligned}
& +2\left(\delta\left(x-y_{1}\right)\right. \\
& \left.+\delta\left(x-y_{2}\right)\right) \mathcal{T}_{5(0,1)}^{\lambda_{1} \rho_{1} \lambda_{2} \rho_{2}}\left(y_{1}, y_{2}\right)=0 \\
& \ldots
\end{aligned}
$$

and

$$
\begin{aligned}
\mathcal{T}_{5(0,0)}(x) \equiv & \left\langle 0\left|T_{5(0,0)}{ }_{\lambda}^{\lambda}(x)\right| 0\right\rangle=0 \\
\mathcal{T}_{5(1,0)}\left(x, x_{1}\right) \equiv & \mathcal{T}_{5(1,0) \lambda}^{\lambda \mu_{1} v_{1}}\left(x, x_{1}\right)+2 \delta\left(x-x_{1}\right) \\
& \times\left\langle 0\left|T_{5(0,0)}^{\mu_{1} v_{1}}\left(x_{1}\right)\right| 0\right\rangle=0 \\
\mathcal{T}_{5(0,1)}\left(x, y_{1}\right) \equiv & \mathcal{T}_{5(0,1) \lambda}^{\lambda \mu_{1} v_{1}}\left(x, y_{1}\right)+2 \delta\left(x-y_{1}\right) \\
& \times\left\langle 0\left|T_{(0,0)}^{\lambda \rho_{1}}\left(y_{1}\right)\right| 0\right\rangle=0 \\
\mathcal{T}_{5(1,1)}\left(x, x_{1}, y_{1}\right) \equiv & \mathcal{T}_{5(1,1) \lambda} \lambda_{\mu_{1} v_{1} \lambda_{1} \rho_{1}}\left(x, x_{1}, y_{1}\right) \\
& +2 \delta\left(x-x_{1}\right) \mathcal{T}_{5(1,0)}^{\mu_{1} v_{1} \lambda_{1} \rho_{1}}\left(x_{1}, y_{1}\right) \\
& +2 \delta\left(x-y_{1}\right) \mathcal{T}_{(0,1)}^{\mu_{1} v_{1} \lambda_{1} \rho_{1}}\left(x_{1}, y_{1}\right)=0
\end{aligned}
$$

$$
\begin{aligned}
\mathcal{T}_{5(2,0)}\left(x, x_{1}, x_{2}\right) \equiv & \mathcal{T}_{5(2,0)}{ }_{\lambda}^{\lambda \mu_{1} v_{1} \mu_{2} \nu_{2}}\left(x, x_{1}, x_{2}\right) \\
& +2\left(\delta\left(x-x_{1}\right)+\delta\left(x-x_{2}\right)\right) \\
& \times \mathcal{T}_{(1,0)}^{\mu_{1} \nu_{1} \mu_{2} \nu_{2}}\left(x_{1}, x_{2}\right)=0,
\end{aligned}
$$

$\mathcal{T}_{5(0,2)}\left(x, y_{1}, y_{2}\right) \equiv \mathcal{T}_{5(0,2) \lambda}{ }^{\lambda \lambda_{1} \rho_{1} \lambda_{2} \rho_{2}}\left(x, y_{1}, y_{2}\right)$

$$
\begin{aligned}
& +2\left(\delta\left(x-y_{1}\right)+\delta\left(x-y_{2}\right)\right) \\
& \times \mathcal{T}_{(0,1)}^{\lambda_{1} \rho_{1} \lambda_{2} \rho_{2}}\left(y_{1}, y_{2}\right)=0, \\
& \ldots
\end{aligned}
$$

where

$$
\begin{aligned}
\mathcal{T}_{(1,0)}^{\mu \nu \mu_{1} \nu_{1}}\left(x, x_{1}\right)= & i\left\langle 0\left|\mathcal{T} T_{(0,0)}^{\mu \nu}(x) T_{(0,0)}^{\mu_{1} \nu_{1}}\left(x_{1}\right)\right| 0\right\rangle \\
& +4\left\langle 0\left|\frac{\delta^{2} S}{\delta h_{\mu \nu}(x) \delta h_{\mu_{1} \nu_{1}}\left(x_{1}\right)}\right| 0\right\rangle, \\
\mathcal{T}_{(0,1)}^{\mu \nu \lambda_{1} \rho_{1}}\left(x, y_{1}\right)= & i\left\langle 0\left|\mathcal{T} T_{5(0,0)}^{\mu \nu}(x) T_{(0,0)}^{\lambda_{1} \rho_{1}}\left(y_{1}\right)\right| 0\right\rangle \\
& +4\left\langle 0\left|\frac{\delta^{2} S}{\delta h_{\mu \nu}(x) \delta k_{\lambda_{1} \rho_{1}}\left(y_{1}\right)}\right| 0\right\rangle
\end{aligned}
$$

and

$$
\begin{aligned}
& \mathcal{T}_{(2,0)}^{\mu \nu \mu_{1} v_{1} \mu_{2} v_{2}}\left(x, x_{1}, x_{2}\right)=-\langle 0| \mathcal{T} T_{(0,0)}^{\mu \nu}(x) \\
& \quad \times T_{(0,0)}^{\mu_{1} v_{1}}\left(x_{1}\right) T_{(0,0)}^{\mu_{2} \nu_{2}}\left(x_{2}\right)|0\rangle \\
& +4 i\left\langle 0\left|\mathcal{T} T_{(0,0)}^{\mu_{1} v_{1}}\left(x_{1}\right) \frac{\delta^{2} S}{\delta h_{\mu \nu}(x) \delta h_{\mu_{2} \nu_{2}}\left(x_{2}\right)}\right| 0\right\rangle \\
& +4 i\left\langle 0\left|\mathcal{T} T_{(0,0)}^{\mu_{2} \nu_{2}}\left(x_{2}\right) \frac{\delta^{2} S}{\delta h_{\mu_{1} v_{1}}\left(x_{1}\right) \delta h_{\mu \nu}(x)}\right| 0\right\rangle \\
& +4 i\left\langle 0\left|\mathcal{T} T_{(0,0)}^{\mu \nu}(x) \frac{\delta^{2} S}{\delta h_{\mu_{1} v_{1}}\left(x_{1}\right) \delta h_{\mu_{2} v_{2}}\left(x_{2}\right)}\right| 0\right\rangle
\end{aligned}
$$

$$
+8\left\langle 0\left|\frac{\delta^{3} S}{\delta h_{\mu \nu}(x) \delta h_{\mu_{1} \nu_{1}}\left(x_{1}\right) h_{\mu_{2} \nu_{2}}\left(x_{2}\right)}\right| 0\right\rangle,
$$

$$
\begin{aligned}
& \mathcal{T}_{(0,2)}^{\mu \nu \lambda_{1} \rho_{1} \lambda_{2} \rho_{2}}\left(x, y_{1}, y_{2}\right)=-\langle 0| \mathcal{T} T_{(0,0)}^{\mu \nu}(x) \\
& \quad \times T_{5(0,0)}^{\lambda_{1} \rho_{1}}\left({ }_{1}\right) T_{5(0,0)}^{\lambda_{2} \rho_{2}}\left(y_{2}\right)|0\rangle \\
& +4 i\left\langle 0\left|\mathcal{T} T_{5(0,0)}^{\lambda_{1} \rho_{1}}\left(y_{1}\right) \frac{\delta^{2} S}{\delta h_{\mu \nu}(x) \delta k_{\lambda_{2} \rho_{2}}\left(y_{2}\right)}\right| 0\right\rangle \\
& +4 i\left\langle 0\left|\mathcal{T} T_{5(0,0)}^{\lambda_{2} \rho_{2}}\left(y_{2}\right) \frac{\delta^{2} S}{\delta k_{\lambda_{1} \rho_{1}}\left(y_{1}\right) \delta h_{\mu \nu}(x)}\right| 0\right\rangle \\
& +4 i\left\langle 0\left|\mathcal{T} T_{(0,0)}^{\mu \nu}(x) \frac{\delta^{2} S}{\delta k_{\lambda_{1} \rho_{1}}\left(y_{1}\right) \delta k_{\lambda_{2} \rho_{2}}\left(y_{2}\right)}\right| 0\right\rangle \\
& +8\left\langle 0\left|\frac{\delta^{3} S}{\delta h_{\mu \nu}(x) \delta k_{\lambda_{1} \rho_{1}}\left(y_{1}\right) h_{\lambda_{2} \rho_{2}}\left(y_{2}\right)}\right| 0\right\rangle
\end{aligned}
$$

and

$$
\begin{aligned}
& \mathcal{T}_{(1,1)}^{\mu \nu \mu_{1} \nu_{1} \lambda_{1} \rho_{1}}\left(x, x_{1}, y_{1}\right)=-\langle 0| \mathcal{T} T_{(0,0)}^{\mu \nu}(x) \\
& \quad \times T_{(0,0)}^{\mu_{1} \nu_{1}}\left(x_{1}\right) T_{5(0,0)}^{\lambda_{1} \rho_{1}}\left(y_{1}\right)|0\rangle \\
& +4 i\left\langle 0\left|\mathcal{T} T_{5(0,0)}^{\lambda \rho_{1}}\left(y_{1}\right) \frac{\delta^{2} S}{\delta h_{\mu \nu}(x) \delta h_{\mu_{1} \nu_{1}}\left(x_{1}\right)}\right| 0\right\rangle \\
& +4 i\left\langle 0\left|\mathcal{T} T_{(0,0)}^{\mu_{1} \nu_{1}}\left(x_{1}\right) \frac{\delta^{2} S}{\delta k_{\lambda_{1} \rho_{1}}\left(y_{1}\right) \delta h_{\mu \nu}(x)}\right| 0\right\rangle \\
& +4 i\left\langle 0\left|\mathcal{T} T_{(0,0)}^{\mu \nu}(x) \frac{\delta^{2} S}{\delta k_{\lambda_{1} \rho_{1}}\left(y_{1}\right) \delta h_{\mu_{1} \nu_{1}}\left(x_{1}\right)}\right| 0\right\rangle \\
& +8\left\langle 0\left|\frac{\delta^{3} S}{\delta h_{\mu \nu}(x) \delta h_{\mu_{1} \nu_{1}}\left(x_{1}\right) k_{\lambda_{1} \rho_{1}}\left(y_{1}\right)}\right| 0\right\rangle
\end{aligned}
$$

and for the axial tensors

$$
\begin{aligned}
\mathcal{T}_{5(1,0)}^{\lambda \rho \mu_{1} \nu_{1}}\left(x, x_{1}\right)= & i\left\langle 0\left|\mathcal{T} T_{5(0,0)}^{\lambda \rho}(x) T_{(0,0)}^{\mu_{1} \nu_{1}}\left(x_{1}\right)\right| 0\right\rangle \\
& +4\left\langle 0\left|\frac{\delta^{2} S}{\delta k_{\lambda \rho}(x) \delta h_{\mu_{1} v_{1}}\left(x_{1}\right)}\right| 0\right\rangle, \\
\mathcal{T}_{5(0,1)}^{\lambda \rho \lambda_{1} \rho_{1}}\left(x, y_{1}\right)= & i\left\langle 0\left|\mathcal{T} T_{5(0,0)}^{\lambda \rho}(x) T_{(0,0)}^{\lambda_{1} \rho_{1}}\left(y_{1}\right)\right| 0\right\rangle \\
& +4\left\langle 0\left|\frac{\delta^{2} S}{\delta k_{\lambda \rho}(x) \delta k_{\lambda_{1} \rho_{1}}\left(y_{1}\right)}\right| 0\right\rangle,
\end{aligned}
$$

$$
\begin{aligned}
& \mathcal{T}_{5(1,1)}^{\lambda \rho \mu_{1} v_{1} \lambda_{1} \rho_{1}}\left(x, x_{1}, y_{1}\right)=-\langle 0| \mathcal{T} T_{5(0,0)}^{\lambda \rho}(x) \\
& \quad \times T_{(0,0)}^{\mu_{1} \nu_{1}}\left(x_{1}\right) T_{5(0,0)}^{\lambda_{1} \rho_{1}}\left(y_{1}\right)|0\rangle \\
& +4 i\left\langle 0\left|\mathcal{T} T_{5(0,0)}^{\lambda \rho}(x) \frac{\delta^{2} S}{\delta h_{\mu_{1} \nu_{1}}\left(x_{1}\right) \delta k_{\lambda_{1} \rho_{1}}\left(y_{1}\right)}\right| 0\right\rangle \\
& +4 i\left\langle 0\left|\mathcal{T} T_{(0,0)}^{\mu_{1} v_{1}}\left(x_{1}\right) \frac{\delta^{2} S}{\delta k_{\lambda_{1} \rho_{1}}\left(y_{1}\right) \delta k_{\lambda \rho}(x)}\right| 0\right\rangle \\
& +4 i\left\langle 0\left|\mathcal{T} T_{5(0,0)}^{\lambda \rho}(x) \frac{\delta^{2} S}{\delta k_{\lambda_{1} \rho_{1}}\left(y_{1}\right) \delta h_{\mu_{1} v_{1}}\left(x_{1}\right)}\right| 0\right\rangle \\
& +8\left\langle 0\left|\frac{\delta^{3} S}{\delta h_{\lambda \rho}(x) \delta h_{\mu_{1} v_{1}}\left(x_{1}\right) h_{\lambda_{1} \rho_{1}}\left(y_{1}\right)}\right| 0\right\rangle
\end{aligned}
$$


and

$$
\begin{aligned}
& \mathcal{T}_{5(2,0)}^{\lambda \rho \mu_{1} \nu_{1} \mu_{2} \nu_{2}}\left(x, x_{1}, x_{2}\right)=-\langle 0| \mathcal{T} T_{5(0,0)}^{\lambda \rho}(x) \\
& \quad \times T_{(0,0)}^{\mu_{1} \nu_{1}}\left(x_{1}\right) T_{(0,0)}^{\mu_{2} \nu_{2}}\left(x_{2}\right)|0\rangle \\
& +4 i\left\langle 0\left|\mathcal{T} T_{(0,0)}^{\mu_{1} \nu_{1}}\left(x_{1}\right) \frac{\delta^{2} S}{\delta k_{\lambda \rho}(x) \delta h_{\mu_{2} \nu_{2}}\left(x_{2}\right)}\right| 0\right\rangle \\
& +4 i\left\langle 0\left|\mathcal{T} T_{(0,0)}^{\mu_{2} \nu_{2}}\left(x_{2}\right) \frac{\delta^{2} S}{\delta h_{\mu_{1} \nu_{1}}\left(x_{1}\right) \delta k_{\lambda \rho}(x)}\right| 0\right\rangle \\
& +4 i\left\langle 0\left|\mathcal{T} T_{5(0,0)}^{\lambda \rho}(x) \frac{\delta^{2} S}{\delta h_{\mu_{1} \nu_{1}}\left(x_{1}\right) \delta h_{\mu_{2} \nu_{2}}\left(x_{2}\right)}\right| 0\right\rangle \\
& +8\left\langle 0\left|\frac{\delta^{3} S}{\delta k_{\lambda \rho}(x) \delta h_{\mu_{1} \nu_{1}}\left(x_{1}\right) h_{\mu_{2} \nu_{2}}\left(x_{2}\right)}\right| 0\right\rangle
\end{aligned}
$$

and

$$
\begin{aligned}
& \mathcal{T}_{5(0,2)}^{\lambda \rho \lambda_{1} \rho_{1} \lambda_{2} \rho_{2}}\left(x, y_{1}, y_{2}\right)=-\langle 0| \mathcal{T} T_{5(0,0)}^{\lambda \rho}(x) \\
& \quad \times T_{5(0,0)}^{\lambda_{1} \rho_{1}}\left(y_{1}\right) T_{5(0,0)}^{\lambda_{2} \rho_{2}}\left(y_{2}\right)|0\rangle \\
& +4 i\left\langle 0\left|\mathcal{T} T_{5(0,0)}^{\lambda_{1} \rho_{1}}\left(y_{1}\right) \frac{\delta^{2} S}{\delta k_{\lambda \rho}(x) \delta k_{\lambda_{2} \rho_{2}}\left(y_{2}\right)}\right| 0\right\rangle \\
& +4 i\left\langle 0\left|\mathcal{T} T_{5(0,0)}^{\lambda_{2} \rho_{2}}\left(y_{2}\right) \frac{\delta^{2} S}{\delta k_{\lambda_{1} \rho_{1}}\left(y_{1}\right) \delta k_{\lambda \rho}(x)}\right| 0\right\rangle \\
& +4 i\left\langle 0\left|\mathcal{T} T_{5(0,0)}^{\lambda \rho}(x) \frac{\delta^{2} S}{\delta k_{\lambda_{1} \rho_{1}}\left(y_{1}\right) \delta k_{\lambda_{2} \rho_{2}}\left(y_{2}\right)}\right| 0\right\rangle \\
& +4\left\langle 0\left|\frac{\delta^{3} S}{\delta k_{\lambda \rho}(x) \delta k_{\lambda_{1} \rho_{1}}\left(y_{1}\right) k_{\lambda_{2} \rho_{2}}\left(y_{2}\right)}\right| 0\right\rangle .
\end{aligned}
$$

\section{Samples of Feynman diagram calculations}

In this appendix we give more details on some of the Feynman diagrams computed in Sect. 3.

\section{C.1 $T_{(0)}$ two-point function}

Let us start from a very simple one, the calculation of $\left\langle 0\left|\mathcal{T} T_{(0)}^{\mu \nu}(x) T_{(0)}^{\lambda \rho}(y)\right| 0\right\rangle$. In momentum space this corresponds to

$$
\begin{aligned}
- & \frac{1}{64} \int \frac{\mathrm{d}^{4} p}{(2 \pi)^{4}} \operatorname{tr}\left(\frac{1}{\not p}(2 p+k)^{\mu} \gamma^{\nu}\right. \\
& \left.\frac{1}{\not p+\not k}(2 p+k)^{\lambda} \gamma^{\rho} \frac{1+\gamma_{5}}{2}\left(\begin{array}{l}
\mu \leftrightarrow v \\
\lambda \leftrightarrow \rho
\end{array}\right)\right),
\end{aligned}
$$

whose odd parity part is

$$
\begin{aligned}
&- \frac{i}{36} \int \frac{\mathrm{d}^{4} p}{(2 \pi)^{4}}\left(\epsilon^{\sigma \nu \tau \rho} \frac{p_{\sigma} k_{\tau}(2 p+k)^{\mu}(2 p+k)^{\lambda}}{p^{2}(p+k)^{2}}\right. \\
&\left.+\left(\begin{array}{c}
\mu \leftrightarrow v \\
\lambda \leftrightarrow \rho
\end{array}\right)\right) .
\end{aligned}
$$

The corresponding regulated expression is

$$
\begin{aligned}
- & \frac{i}{36} \int \frac{\mathrm{d}^{4} p}{(2 \pi)^{4}} \int \frac{d^{\delta} \ell}{(2 \pi)^{\delta}}\left(\epsilon^{\sigma \nu \tau \rho} \frac{p_{\sigma} k_{\tau}(2 p+k)^{\mu}(2 p+k)^{\lambda}}{\left(p^{2}-\ell^{2}\right)\left((p+k)^{2}-\ell^{2}\right)}\right. \\
+ & \left.\left(\begin{array}{c}
\mu \leftrightarrow \nu \\
\lambda \leftrightarrow \rho
\end{array}\right)\right) .
\end{aligned}
$$

Only the terms quadratic in $p$ in the numerator may survive for symmetry reasons, but for the same reason they give rise to $\delta_{\sigma}^{\mu}$ and $\delta_{\sigma}^{\lambda}$, which leads to the vanishing of (287). If we contract (287) with $\eta_{\mu \nu}$ its vanishing is even more evident.

\section{C.1.1 Terms $P-V_{f f h}-P-V_{f f h h}^{\prime}$ and similar}

We wish to evaluate the terms contained in $\langle 0| \mathcal{T} T_{(0)}^{\mu \nu}(x)$ $\frac{\delta^{2} S}{\delta h_{\mu_{1} v_{1}}\left(x_{1}\right) \delta h_{\mu_{2} v_{2}}\left(x_{2}\right)}|0\rangle$. They are diagram with an incoming graviton line of momentum $q$ and two outgoing ones of momentum $k_{1}, k_{2}$. The first is the diagram $P-V_{f f h}-P-$ $V_{f f h h}^{\prime}$, whose odd part is

$$
\begin{aligned}
& \frac{3}{512} \int \frac{\mathrm{d}^{4} p}{(2 \pi)^{4}}\left[\left(\left(\frac{1}{\not p}(2 p-q)^{\mu} \gamma^{\nu} \frac{1}{\not p-q}(2 p-q)^{\mu_{1}} \gamma^{\nu_{1}} \eta^{\nu_{1} \nu_{2}}\right.\right.\right. \\
& \left.\left.\left.+\left(\begin{array}{c}
\mu \leftrightarrow v \\
\mu_{1} \leftrightarrow v_{1} \\
\mu_{2} \leftrightarrow v_{2}
\end{array}\right)\right)\left(\mu_{1}, \nu_{1}\right) \leftrightarrow\left(\mu_{2}, \nu_{2}\right)\right) \frac{\gamma_{5}}{2}\right] .
\end{aligned}
$$

Saturating it with $\eta_{\mu \nu}$ one gets

$$
\begin{aligned}
& \frac{3}{512} \int \frac{\mathrm{d}^{4} p}{(2 \pi)^{4}} \operatorname{tr}\left[\left(\frac{1}{\not p}(2 \not p-q) \frac{1}{\not p-q}(2 p-q)^{\mu_{1}} \gamma^{\nu_{1}} \eta^{\nu_{1} \nu_{2}}\right.\right. \\
& \left.+\left(\begin{array}{l}
\mu_{1} \leftrightarrow \nu_{1} \\
\mu_{2} \leftrightarrow \nu_{2}
\end{array}\right)\right) \\
& \left.\left.+\left(\mu_{1}, \nu_{1}\right) \leftrightarrow\left(\mu_{2}, \nu_{2}\right)\right) \frac{\gamma_{5}}{2}\right]
\end{aligned}
$$

which clearly vanishes because of the $\gamma$ trace. It follows that also the odd part of the diagram $P-V_{f f h}^{\prime}-P-V_{f f h h}^{\prime}$ vanishes.

The same conclusion holds if in these previous diagrams we replace $V_{f f h h}^{\prime}$ with $V_{f f h h}^{\prime \prime}$ and $V_{f f h h}^{\prime \prime \prime}$.

Proceeding in the same way we can prove that also the odd part of

$\eta_{\mu \nu}\left\langle 0\left|\mathcal{T} T_{(0)}^{\mu_{1} \nu_{1}}\left(x_{1}\right) \frac{\delta^{2} S}{\delta h_{\mu \nu}(x) \delta h_{\mu_{2} \nu_{2}}\left(x_{2}\right)}\right| 0\right\rangle$

vanishes. But there is a simpler way to get rid of the terms containing one $T_{(0)}$ factor and one second derivative of $S$ and it is to prove that their odd parity part vanishes before taking the trace.

Let us consider again (288), that is, the untraced $P_{-}$ $V_{f f h}-P-V_{f f h h}^{\prime}$. Introducing a dimensional regulator $\delta$ we 
can rewrite it as

$$
\begin{aligned}
& \frac{3}{1024} \int \frac{\mathrm{d}^{4} p}{(2 \pi)^{4}} \int \frac{d^{\delta} \ell}{(2 \pi)^{\delta}} \\
& \times\left[\operatorname{tr}\left(\frac{\not p}{p^{2}-\ell^{2}} \gamma_{\nu} \frac{\not p-q}{(p-q)^{2}-\ell^{2}} \gamma_{\mu_{2}} \gamma_{5}\right)\right. \\
& \left.\times(2 p-q)^{\mu}(2 p-q)^{\mu_{1}} \eta^{\nu_{1} \nu_{2}}+\cdots\right]
\end{aligned}
$$

where the dots denote the symmetrizations indicated in (288). Let us take the $\gamma$ trace:

$$
\begin{aligned}
& \frac{3 i}{256} \int \frac{\mathrm{d}^{4} p}{(2 \pi)^{4}} \int \frac{d^{\delta} \ell}{(2 \pi)^{\delta}} \epsilon^{\sigma \nu \tau \mu_{2}} p_{\sigma} q_{\tau} \\
& \frac{(2 p-q)^{\mu}(2 p-q)^{\mu_{1}} \eta^{\nu_{1} \nu_{2}}}{\left(p^{2}-\ell^{2}\right)\left((p-q)^{2}-\ell^{2}\right)}+\cdots .
\end{aligned}
$$

The integrand has two $p^{2}$ terms in the numerator. They are proportional, respectively, to $\epsilon^{\mu \nu \tau \mu_{2}} q_{\tau} q^{\mu_{1}} \eta^{\nu_{1} \nu_{2}}$ and $\epsilon^{\mu_{1} v \tau \mu_{2}} q_{\tau} q^{\mu} \eta^{\nu_{1} \nu_{2}}$. The first vanishes under the $\mu \leftrightarrow \nu$ symmetrization, the other under the symmetrization $\left(\mu_{1}, v_{1}\right) \leftrightarrow$ $\left(\mu_{2}, v_{2}\right)$.

Next we do the same for the untraced $P-V_{f f h}-P-V_{f f h h}^{\prime \prime \prime}$. The relevant integral is

$$
\begin{aligned}
& \frac{1}{128} \int \frac{\mathrm{d}^{4} p}{(2 \pi)^{4}} \operatorname{tr}\left[\frac{1}{\not p}(2 p-q)^{\mu} \gamma^{\nu} \frac{1}{\not p-q}(2 \not p-q) \frac{\gamma_{5}}{2}\right. \\
& \left.\times\left(\eta^{\mu_{1} \nu_{1}} \eta^{\mu_{2} \nu_{2}}-\eta^{\mu_{1} \nu_{2}} \eta^{\mu_{2} \nu_{1}}-\eta^{\mu_{1} \mu_{2}} \eta^{\nu_{1} \nu_{2}}\right)\right]
\end{aligned}
$$

symmetrized in $\mu \leftrightarrow v$. Writing $2 \not p-q=\not p+\not p-q$ and simplifying with the denominators, we get two terms each with a trace of two $\gamma$ 's with $\gamma_{5}$, which vanishes.

\section{C.1.2 The term $P-V_{f f h}-P-V_{f f h h}^{\epsilon}$}

This term requires a bit more elaboration. The starting point is the integral

$$
\begin{aligned}
& \frac{i}{512} \int \frac{\mathrm{d}^{4} p}{(2 \pi)^{4}} \operatorname{tr}\left[\frac{1}{\not p}(2 p-q)^{\mu} \gamma^{\nu} \frac{1}{\not p-q} t^{\mu_{1} \nu_{1} \mu_{2} \nu_{2} \kappa \lambda}\right. \\
& \left.\quad \times\left(k_{1}-k_{2}\right)_{\lambda} \gamma_{\kappa} \frac{1+\gamma_{5}}{2}\right],
\end{aligned}
$$

which has to be symmetrized in $\mu \leftrightarrow \nu$. The odd part is

$$
\begin{aligned}
& \frac{i}{1024} \int \frac{\mathrm{d}^{4} p}{(2 \pi)^{4}}\left[\operatorname{tr}\left(\frac{1}{\not p} \gamma^{\nu} \frac{1}{\not p-q} \gamma_{\kappa}\right)(2 p-q)^{\mu} t^{\mu_{1} \nu_{1} \mu_{2} \nu_{2} \kappa \lambda}\right. \\
& \left.\times\left(k_{1}-k_{2}\right)_{\lambda}+(\mu \rightarrow \nu)\right] .
\end{aligned}
$$

Next we introduce the dimensional regulator and use Lorentz covariance to obtain

$$
\begin{aligned}
& \frac{i}{256} \int \frac{\mathrm{d}^{4} p}{(2 \pi)^{4}} \int \frac{d^{\delta} \ell}{(2 \pi)^{\delta}} \frac{1}{\left(p^{2}-\ell^{2}\right)\left((p-q)^{2}-\ell^{2}\right)} \times \\
& \quad \times\left[\left(p^{\nu}(p-q)_{\kappa}-\left(p \cdot(p-q)+\ell^{2}\right) \delta_{\kappa}^{v}+p_{\kappa}(p-q)^{v}\right)\right. \\
& \left.\quad \times(2 p-q)^{\mu} t^{\mu_{1} \nu_{1} \mu_{2} \nu_{2} \kappa \lambda}\left(k_{1}-k_{2}\right)_{\lambda}+(\mu \rightarrow v)\right] .
\end{aligned}
$$

Next we introduce a Feynman parameter $x, 0 \leq x \leq 1$ and represent

$$
\begin{aligned}
& \frac{1}{\left(p^{2}-\ell^{2}\right)\left((p-q)^{2}-\ell^{2}\right)} \\
& \quad=\int_{0}^{1} \mathrm{~d} x \frac{1}{\left((p-x q)^{2}-\ell^{2}+x(1-x) q^{2}\right)^{2}}
\end{aligned}
$$

then we change variable $p \rightarrow p^{\prime}=p-x q$. The result is

$$
\begin{aligned}
& \frac{i}{256} \int \frac{\mathrm{d}^{4} p}{(2 \pi)^{4}} \int \frac{d^{\delta} \ell}{(2 \pi)^{\delta}} \int_{0}^{1} \mathrm{~d} x \frac{t^{\mu_{1} \nu_{1} \mu_{2} \nu_{2} \kappa \lambda}\left(k_{1}-k_{2}\right)_{\lambda}}{\left(p^{2}-\ell^{2}+x(1-x) q^{2}\right)^{2}} \\
& \quad \times\left[\frac{1}{2}\left(\eta^{\mu \nu} q_{\kappa}+\delta_{\kappa}^{\mu} q^{\nu}+\delta_{\kappa}^{v} q^{\mu}\right) p^{2}(2 x-1)\right. \\
& +\left(2 q^{\mu} q^{\nu} q_{\kappa}-q^{\mu} q^{2} \delta_{\kappa}^{v}\right) x(1-x)(1-2 x) \\
& \left.\quad-\frac{3}{2} q^{\mu} p^{2} \delta_{\kappa}^{\nu}(2 x-1)-\ell^{2} q^{\mu} \delta_{\kappa}^{\nu}(2 x-1)\right]
\end{aligned}
$$

which must be symmetrized under $\mu \leftrightarrow v$. All the terms vanish because of the $x$ integration.

\section{References}

1. L. Bonora, S. Giaccari, B. Lima de Souza, Trace anomalies in chiral theories revisited. JHEP 1407, 117 (2014). arXiv:1403.2606 [hep-th]

2. L. Bonora, A.D. Pereira, B.L. de Souza, Regularization of energymomentum tensor correlators and parity-odd terms. JHEP 1506, 024 (2015). arXiv: 1503.03326 [hep-th]

3. F. Bastianelli, R. Martelli, On the trace anomaly of a Weyl fermion. JHEP 1611, 178 (2016). doi:10.1007/JHEP11(2016)178. arXiv: 1610.02304 [hep-th]

4. W.A. Bardeen, Anomalous ward identities in spinor field theories. Phys. Rev. 184, 1848 (1969)

5. T. Kimura, Divergence of axial-vector current in the gravitational field. Prog. Theor. Phys. 42, 1191 (1969)

6. R. Delbourgo, A. Salam, PCAC anomalies and gravitation. Preprint IC/72/86

7. R. Delbourgo, A. Salam, The gravitational correction to PCAC. Phys. Lett. 40B, 381 (1972)

8. S. Mauro, I.L. Shapiro, Anomaly-induced effective action and Chern-Simons modification of general relativity. Phys. Lett. B 746, 372 (2015). doi:10.1016/j.physletb.2015.05.045. arXiv:1412.5002 [gr-qc]

9. G. Racah, On the symmetry of particles and antiparticles. Nuovo Cimento 14, 322 (1936)

10. J.F. Cornwell, Group Theory in Physics, vol. II, Ch. 17 (Academic Press, London, 1984)

11. P.B. Pal, Dirac, Majorana and Weyl fermions. arXiv: 1006.1718 [hep-th] 
12. A. Andrianov, L. Bonora, Finite-mode regularization of the fermion functional integral. II. Nucl. Phys. B 233, 247 (1984)

13. A. Andrianov, L. Bonora, Finite-mode regularization of the fermion functional integral. I. Nucl. Phys. B 233, 232 (1984)

14. D.M. Grabowska, D.B. Kaplan, Chiral solution to the GinspargWilson equation. Phys. Rev. D 94(11), 114504 (2016). doi:10. 1103/PhysRevD.94.114504. arXiv:1610.02151 [hep-lat]

15. R.A. Bertlmann, Anomalies in Quantum Field Theory (Oxford Science Publications, Oxford, 1996)

16. K. Fujikawa, H. Suzuki, Path Integrals and Quantum Anomalies (Oxford Science Publications, Oxford, 2004)

17. F. Bastianelli, P. Van Nieuwenhuizen, Path Integrals and Anomalies in Curved Space (Cambridge University Press, Cambridge, 2009)

18. L.E. Parker, Quantum Field Theory in Curved Spacetime: Quantized Fields and Gravity (Cambridge University Press, Cambridge, 2009)
19. L. Bonora, P. Pasti, M. Tonin, Gravitational and Weyl anomalies. Phys. Lett. 149B, 346 (1985)

20. L. Bonora, P. Pasti, M. Tonin, The anomaly structure of theories with external gravity. J. Math. Phys. 27, 2259 (1986)

21. L. Bonora, M. Cvitan, P. Dominis Prester, S. Giaccari, B. Lima de Souza, T. Stemberga, One-loop effective actions and higher spins. arXiv:1609.02088 [hep-th]. To appear in JHEP

22. L. Bonora, M. Bregola, P. Pasti, Weyl cocycles. Class. Quant. Gravity 3, 635 (1986)

23. S.M. Christensen, M.J. Duff, New gravitational index theorems and super theorems. Nucl. Phys. B 154, 301 (1979)

24. Y. Nakayama, CP-violating CFT and trace anomaly. Nucl. Phys. B 859, 288 (2012)

25. L. Bonora, B.L. de Souza, Pure contact term correlators in CFT. Proc. 18th Bled Workshop "What Comes Beyond Standard Models", Bled 2015. arXiv:1511.06635 [hep-th] 